\title{
Ring Opening of Triflates Derived from Benzophospholan-3-one Oxides by Aryl Grignard Reagents as a Route to 2-Ethynylphenyl(diaryl)phosphine Oxides
}

\author{
Łukasz Ponikiewski and Sylwia Sowa*
}

Cite This: J. Org. Chem. 2021, 86, 14928-14941

Read Online

\section{ACCESS |}

Џll Metrics \& More

回国 Article Recommendations

S1. Supporting Information

ABSTRACT: A new simple method for the synthesis of 2-ethynylphenyl(diaryl)phosphine oxides via ring opening of benzophosphol-3-yl triflates has been developed. This process occurs via nucleophilic attack of a Grignard reagent at the phosphorus center, which results in ring opening and cleavage of a leaving group. The reaction proceeds under mild conditions and, within 15-60 min, leads to a library of previously unavailable 2-ethynylphenylphosphine oxides in yields up to $98 \%$.

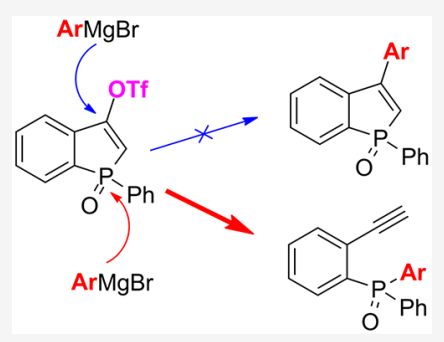

\section{INTRODUCTION}

Compounds possessing an ethynyl fragment became an attractive pattern in modern organic chemistry. ${ }^{1}$ The growing importance of the chemistry of carbon-carbon triple bonds is due to their unique and diverse chemical reactivity and remarkable synthetic potential as they can be used as precursors for many classes of compounds including alkanes, alkenes, aldehydes, and carboxylic acids on both laboratory and industrial scales. ${ }^{1,2}$ Recently, ethynyl compounds have been intensively explored in catalytic reactions mediated by different metals (gold, ${ }^{3}$ copper, ${ }^{4}$ palladium, ${ }^{5}$ rhodium, ${ }^{6}$ and others ${ }^{7}$ ), providing various cyclic products. Moreover, ethynyl compounds have a wide range of applications in the synthesis of biologically active compounds and have been broadly exploited in drug discovery and development. ${ }^{8}$ Furthermore, an alkyne functionality is frequently used in medicine ${ }^{9}$ and chemical biology ${ }^{10}$ as a "click" probe to label or identify molecular targets and to assess target engagement. The "click-type" reactions of ethynyl compounds have also been exploited to synthesize $\pi$-conjugated macromolecules for organic electronics $^{11}$ and other materials. ${ }^{12}$ On the other hand, ethynyl compounds themselves, especially those possessing expanded $\pi$-systems, have been involved in the preparation of organic structures for photo-optical purposes and others. ${ }^{13}$ All mentioned applications of alkynes benefit from the rapid development of the synthetic routes, enabling the incorporation of an ethynyl unit into the structure of nearly any organic molecule. The most explored methods here are Sonogashira coupling, ${ }^{14}$ copper-free Sonogashira reactions, ${ }^{15}$ and alkynylation reaction. ${ }^{16}$

In the field of organophosphorus chemistry, (ethynyl)phosphine oxides were employed in the preparations of socalled "smart" polymers ${ }^{17}$ but also as the starting materials for metal-catalyzed coupling, ${ }^{18}$ cyclization, ${ }^{19}$ cycloaddition, ${ }^{20}$ and radical addition/cycloaddition. ${ }^{21}$ Another class of organophosphorus-possessing $\mathrm{C}-\mathrm{C}$ triple bond compounds mentioned in the literature is $p$-ethynylphenylphosphine oxides. Those compounds have found several applications in the construction of star-shaped fluorescent molecules, ${ }^{22}$ microporous organic polymers (MOPs), ${ }^{23}$ nanostructured carbon materials, ${ }^{24}$ and triazole precursors. ${ }^{25}$ Their ortho analogues with phosphorus at a lower oxidation state are typically used for the construction of the benzophosphole skeleton. ${ }^{26}$ The access to those derivatives was achieved via a reaction of $o$ lithiotolane with chlorophosphine (Scheme 1A). ${ }^{26 e, 27} \mathrm{~A}$ different route to these derivatives was completed through Sonogashira coupling. ${ }^{28}$ In 2020, Duan et al. showed that methyl(phenyl)phosphinic chloride in a reaction with lithium $o$-phenylethynylbenzene had provided o-phenylethynylphosphine oxide, which is immediately transformed into the corresponding benzophosphole oxide (Scheme 1B). ${ }^{29}$ Another compound possessing an ethynyl moiety in the ortho position was obtained via $[2+2+2]$ cyclization (Scheme $1, \mathrm{C}) .^{30}$ To the best of our knowledge, the phosphine oxides possessing a terminal ethynyl moiety in the ortho position are unknown. The closest known analogues are derivatives of (oethynylphenyl)phosphonic acid synthesized by $\mathrm{Ding}^{31}$ and later Peng. ${ }^{32}$

Received: July 14, 2021

Published: October 26, 2021 
Scheme 1. Examples of the Synthesis of 2Ethynylphenylphosphine Derivatives

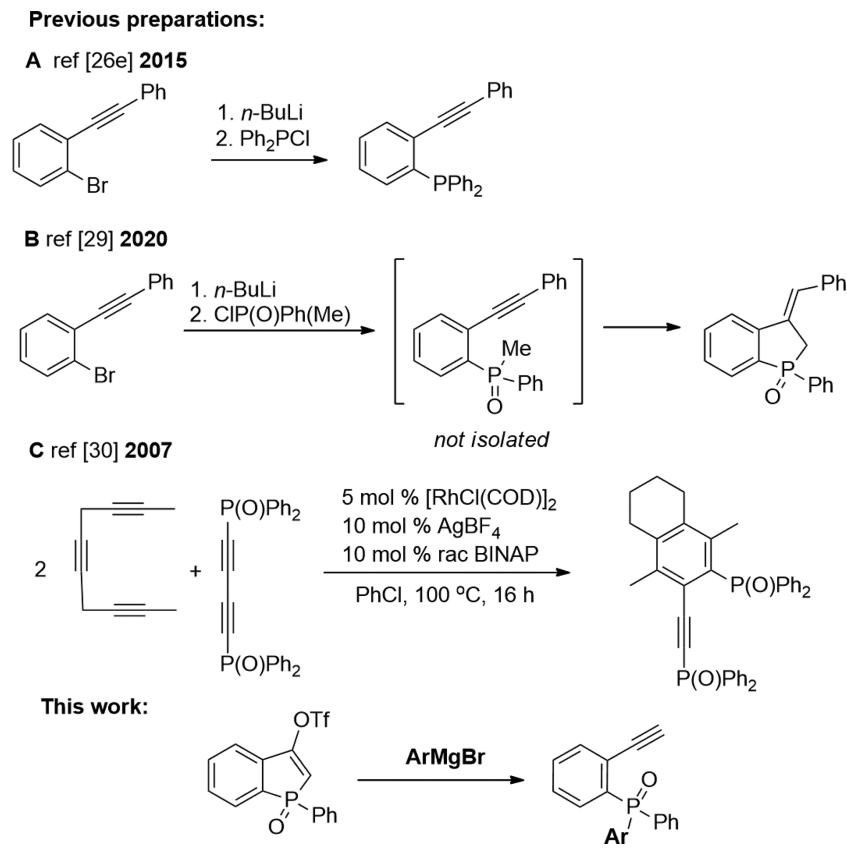

\section{RESULTS AND DISCUSSION}

Herein, we want to present a new route to 2-ethynylphenyl(diaryl)phosphine oxides via unprecedented ring opening of triflates derived from benzophospholan-3-one oxides.

We began our study with the preparation of a set of triflates 5 and 6 from benzophospholan-3-one oxides 3 and benzophospholan-3-one sulfide 4a (Scheme 2, path $d$ ). Benzophospholan-3-one oxides 3 and sulfide $4 a$, which were starting materials for triflates $\mathbf{5}$ and $\mathbf{6 a}$, were prepared in a twostep procedure, utilizing Ullman type coupling of phosphine oxide 1 followed by cyclization in the presence of LDA (Scheme 2, paths a and b). The preparation of benzophosphole sulfide $\mathbf{4 a}$ was achieved via chemoselective reduction of $\mathrm{P}=\mathrm{O}$ in 3 followed by oxidation of the phosphorus center with sulfur (Scheme 2, path c).

Our primary synthetic goal was to investigate the reactivity of triflates $\mathbf{5}$ and $\mathbf{6 a}$ toward aryl Grignard reagents in order to prepare 3-arylbenzophosphole oxides 7 according to the reaction depicted in Scheme 3.

Scheme 3. Predicted Outcome of the Reaction of 5a with Aryl Grignard Reagents

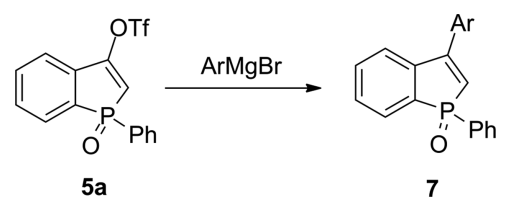

In order to check the reactivity of triflates toward the Grignard reagent, the model triflate 5a was reacted with $p$ tolylmagnesium bromide (Table 1). To our surprise, instead of

Table 1. Optimization of Reaction Conditions in a Reaction of $5 \mathrm{a}$ and $p$-TolMgBr ${ }^{a}$

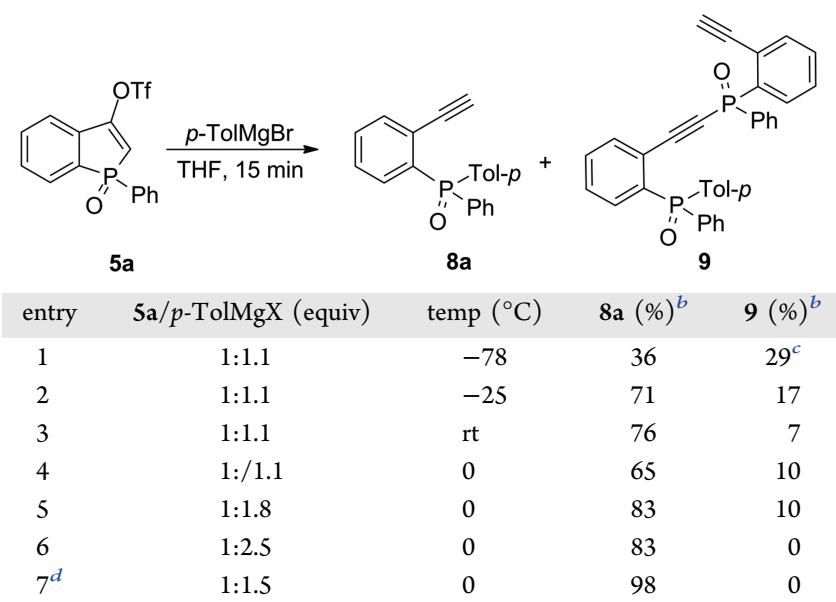

${ }^{a}$ Reaction conditions: $5 \mathrm{a}(0.13 \mathrm{mmol}), \operatorname{ArMgBr}(0.195 \mathrm{mmol})$, THF $(2 \mathrm{~mL}) .{ }^{b}$ Isolated yields. ${ }^{c}$ Starting material 5a and 1-phenylbenzophospholan-3-one oxide (3a) were present in the crude reaction mixture. ${ }^{d}$ Reaction was carried out for $30 \mathrm{~min}$.

the expected 1-phenyl-3-( $p$-tolyl)benzophosphole oxide (7a), we have observed compound $\mathbf{8 a}$ (as the major product), which, in most cases, remained in the mixture with diphosphorus compound 9 (Table 1, entries 1-5). We

Scheme 2. Synthetic Route to Triflates 5 and 6

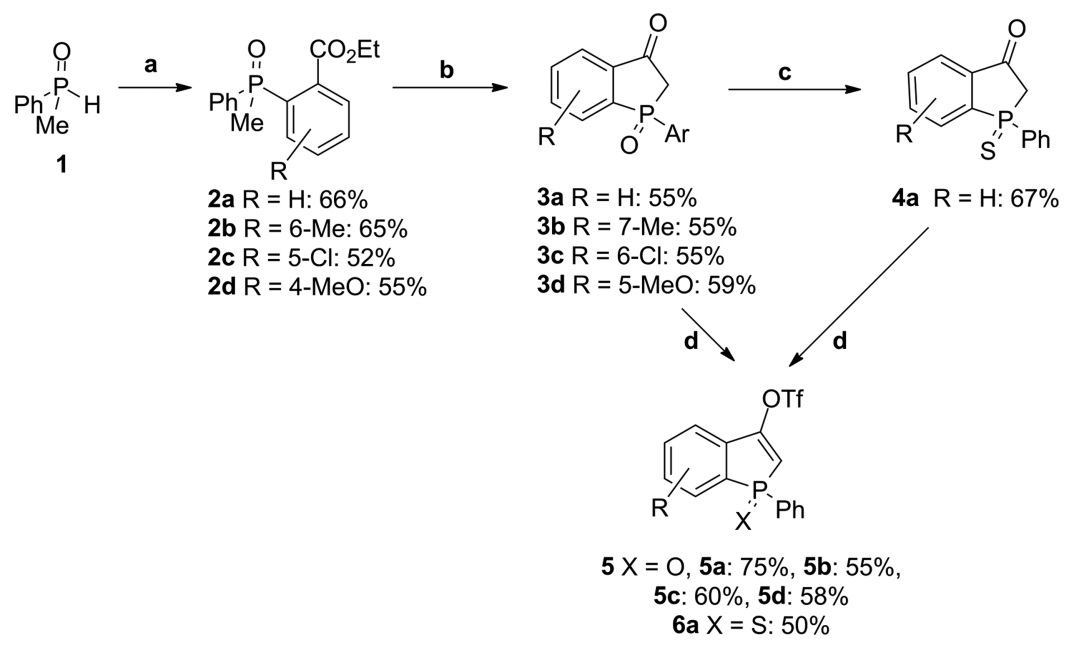




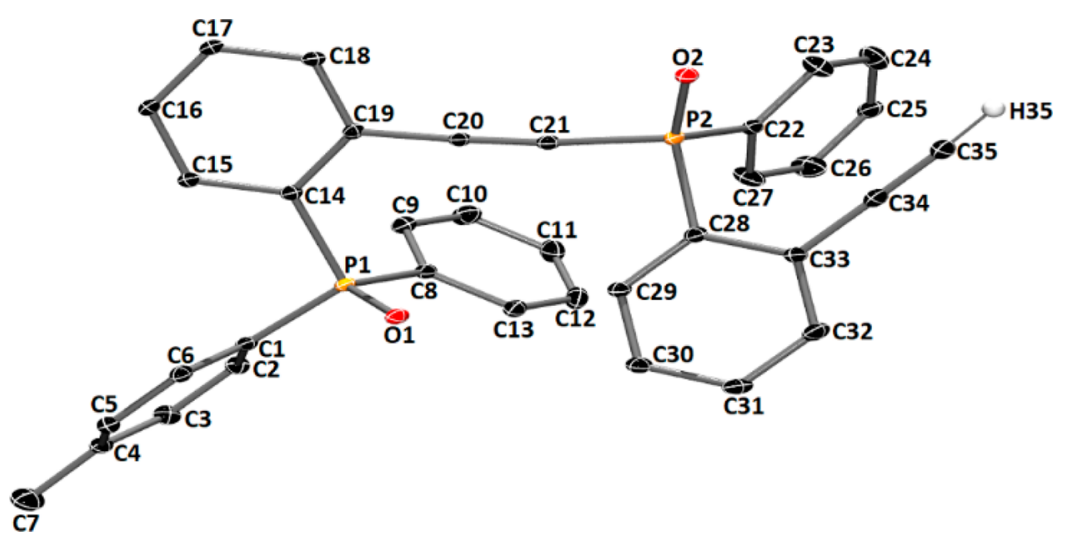

Figure 1. Molecular structure of 9; ellipsoids 30\%, hydrogen atoms have been omitted for clarity (except H35). Important bond distances $(\AA)$, angles (deg) for 9: P1-O1 1.488(2), P2-O2 1.482(2), C20-C21 1.202(4), C34-C35 1.205(6), C35-H35 0.950(2), O1-P1-C1 111.41(14), O1-P1-C8 113.03(15), O1-P1-C14 113.89(15), C20-C21-P2 168.8(3), O2-P2-C22 113.86(16), O2-P2-C28 115.37(15), C33-C34C35 176.0(3).

realized that the aryl Grignard reagent caused the ring opening of the starting triflate 5a, which resulted in the formation of phosphine oxide 8a bearing a 2-ethynylphenyl substituent. Therefore, we decided to focus on the selective transformation of triflates into phosphine oxides 8 possessing a terminal ethynyl group in the ortho position, which gives an opportunity for further functionalization.

First, we screened the influence of the temperature on the reaction of triflate 5a with $p$-TolMgBr (Table 1, entries 1-4). Preliminary attempts showed that the selectivity of the reaction is significantly affected by temperature. When the reaction of 5a was carried out at $-78{ }^{\circ} \mathrm{C}$, a mixture of products was observed in the crude reaction mixture, and the conversion of 5a was not complete (Table 1, entry 1 ). The mixture consisted of $8 \mathbf{a}, 9$, and unreacted $5 \mathbf{a}$ and was accompanied by 1phenylbenzophospholan-3-one oxide (3a). We were able to isolate major product $8 \mathrm{a}$ along with diphosphorus compound 9 in $36 \%$ and $29 \%$ yields, respectively. Under these conditions, selectivity was low, and a relatively high amount of 9 was formed. The structure of compound 9 was confirmed by X-ray analysis (Figure 1). It turned out that compound 9 possesses an alkyne moiety directly connected with the phosphorus atom.

Further attempts have revealed that the full conversion of 5a can be achieved by increasing the temperature (Table 1, entry 2 ). Additionally, the amount of $\mathbf{8 a}$ has significantly increased at $-25{ }^{\circ} \mathrm{C}, 0{ }^{\circ} \mathrm{C}$, and $\mathrm{rt}$ to 71,65 , and $76 \%$ isolated yields, respectively (Table 1 , entries $2-4$ ). However, some amounts of 9 have still been found in the reaction mixture and were isolated in $7-17 \%$ yields. To optimize the conditions, we decided to maintain the temperature of the reaction at $0{ }^{\circ} \mathrm{C}$. A further study has shown that the change in the $\mathbf{5 a} / p$-TolMgBr ratio strongly affected the outcome of the reaction (Table 1 , entries 4-6). In the case where 5a was reacted with 2.5 equiv of the Grignard reagent, desired $\mathbf{8 a}$ was formed as a sole product isolated in $83 \%$ yield (Table 1 , entry 6 ). Finally, the optimal reaction conditions were established by reducing the amount of $p$-TolMgBr and extending the reaction time (Table 1 , entry 7). In this case, the complete conversion of $5 \mathbf{a}$ to $\mathbf{8 a}$ was observed, and $8 \mathbf{a}$ was isolated with the highest yield (98\%).

In the next stage, the reactivity of triflate 5a toward a set of aryl Grignard reagents was checked under the optimized reaction conditions (Table 2). In general, triflate $\mathbf{5 a}$ was
Table 2. Scope of Different ArMgBr Reagents in the Reaction with Triflate $5 a^{a, b, c}$

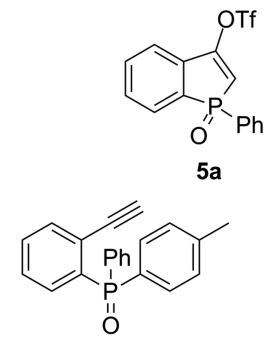

8a 98 (99), 91 (95) ${ }^{d}$
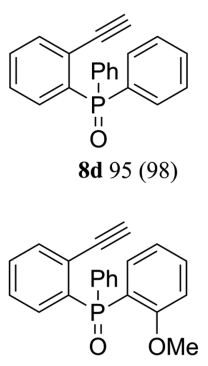

8g 92 (99)

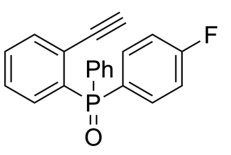

8j 82 (97)

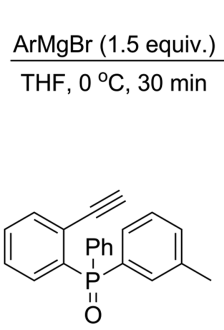

8b 84 (99)

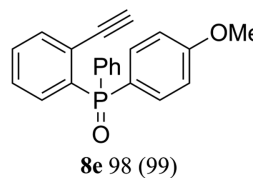

8e 98 (99)
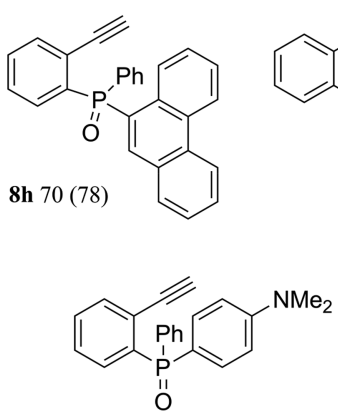

8k $60(90)^{g}$

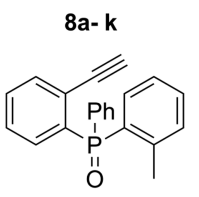

8c $38(68)^{e, f}$
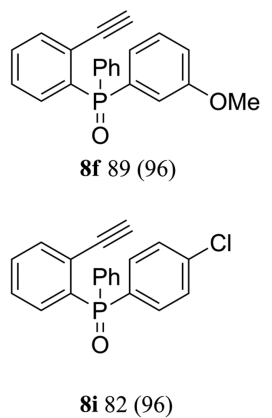

8i 82 (96)

${ }^{a}$ Reaction conditions: 5a $(0.13 \mathrm{mmol}), \operatorname{ArMgBr}(0.195 \mathrm{mmol})$, THF $(2 \mathrm{~mL}){ }^{b}$ Isolated yields of product. ${ }^{c}$ Numbers in parentheses indicate yields according to ${ }^{31} \mathrm{P}$ NMR. ${ }^{d}$ Reaction was carried out on a 0.6 mmol scale. ${ }^{e}$ Reaction was carried out with $o$-TolMgCl. ${ }^{f_{\text {Formation }}}$ of a small amount of ketone $\mathbf{3 a}$ was observed but not isolated.

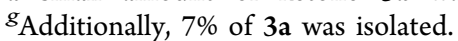

tolerant toward different substitution patterns in the aryl ring of the Grignard reagent used. In most cases, products $8 \mathbf{a}-\mathbf{k}$ were obtained in good to very good yields. In the case of tolyl Grignard reagents, there was no difference between the reactivity of the $p$ - and $m$-tolyl Grignard reagent, and both led to the formation of $\mathbf{8 a}$ and $\mathbf{8 b}$ in $98 \%$ and $84 \%$ yields, 
respectively. In sharp contrast to these reactions, the analogous reaction with $o$-TolMgCl was much less selective and resulted in a mixture of $8 \mathrm{c}$ and ketone $3 \mathrm{a}$. In this case, product $8 \mathrm{c}$ was formed with the lowest yield (38\%). Noteworthy, the reaction with $p$-TolMgBr on a higher scale $(0.6 \mathrm{mmol})$ has also provided desired product $8 \mathbf{a}$ in a high yield $(91 \%)$.

At the same time, the reactivity of triflate 5a toward the Grignard reagent possessing electro-donating substituents (anisylmagnesium bromides) was not greatly dependent on the position of the methoxyl group in the phenyl ring. The reactions of compound $5 \mathbf{a}$ with any isomer $\left(0-, \mathrm{m}^{-}, \mathrm{p}^{-}\right)$of anisylmagnesium bromide provided products $8 \mathbf{d}-\mathbf{f}$ with $89-$ 98\% yields. Another Grignard reagent with an electrondonating substituent, $p-\mathrm{Me}_{2} \mathrm{~N}-\mathrm{C}_{6} \mathrm{H}_{4} \mathrm{MgBr}$, in a reaction with $\mathbf{5 a}$ has provided a mixture of $\mathbf{8 k}$ and $\mathbf{3 a}$, which was enriched in 8k. In this case, 8k and 3a were isolated in 60 and $7 \%$ yields, respectively.

Interestingly, even Grignard reagents possessing halogen atoms in the para position in the phenyl ring formed the desired products with a very high selectivity; however, isolated yields of $\mathbf{8 i}$ and $\mathbf{8 j}$ were slightly lower (82\% yields for both reagents). In turn, a reaction of $\mathbf{5} \mathbf{a}$ under the same conditions with bulky 9-phenantrylmagnesium bromide has led to product $\mathbf{8 h}$ in $70 \%$ yield. Small amounts of other unidentified compounds were also observed but not isolated.

The results achieved for $\mathbf{5 a}$ (Table 2) have prompted us to further investigate the selectivity of this process by changing the substituents in compound $\mathbf{5}$. The obtained results are presented in Table 3. To our delight, once again, we were able to isolate the expected 2-ethynylphenyl(diaryl)phosphine oxides 10-12 in very good yields. In the case of compounds with electron-donating substituents in benzophosphole rings 5b and 5d, a reaction with $\mathrm{PhMgBr}$ afforded $10 \mathrm{~d}$ and $\mathbf{1 2 d}$ in high yields -91 and $82 \%$, respectively. Similar results were obtained when compound $\mathbf{5 c}$ possessing a chlorine substituent was subjected to the reaction with $\mathrm{PhMgBr}$. Product 11d was successfully isolated in a $73 \%$ yield. Moreover, the reactions of 5b with other Grignard reagents ( $p$-TolMgBr, $p$-AnMgBr, $p$ $\mathrm{Cl}-\mathrm{C}_{6} \mathrm{H}_{4} \mathrm{MgBr}$ ) provided $\mathbf{1 0}-\mathbf{1 1} \mathbf{a}, \mathbf{e}, \mathbf{i}$ as the major products in the crude reaction mixture. Therefore, we assume that the studied reaction is tolerant toward differently substituted aryl Grignard reagents. Unfortunately, the isolation step was less successful in comparison to products formed in reactions with $\mathrm{PhMgBr}$ (10d, 11d, 12d). In these cases, we noted lower isolated yields in the range of 58-66\%. Faster isolation of 1012 (higher polarity of the eluent, short chromatography column) resulted in higher yields and moderate purity. In opposite, when compounds were eluted slower (lower polarity of eluent, longer chromatography column), higher purity was noted with a significant reduction of yield. For instance, when isolating 10a in a lower polarity solvent and a higher polarity solvent, we observed $38 \%$ and $66 \%$ yields, respectively. It seems that a terminal acetylene moiety can interact with acidic silica gel, which causes a decrease in yield. Unfortunately, some loss during isolation was also observed for products 10k, 11k, and $12 \mathrm{k}$ possessing an amino moiety. Even the addition of triethylamine to the eluent during the chromatography column was not beneficial, and all of these products were isolated in a moderate yield, $60-70 \%$.

To verify the influence of the $\mathrm{P}=\mathrm{X}$ center on the reaction course, sulfide $\mathbf{6 a}$ derived from 1-phenylbenzophosphole sulfide (4a) was subjected to the reaction with $\mathrm{PhMgBr}$. In contrast to phosphine oxide 5a, phosphine sulfide 6a turned
Table 3. Reactivity of Substituted Benzophosphole Oxides $5 b-d$ with Aryl Grignard Reagents ${ }^{a, b, c}$

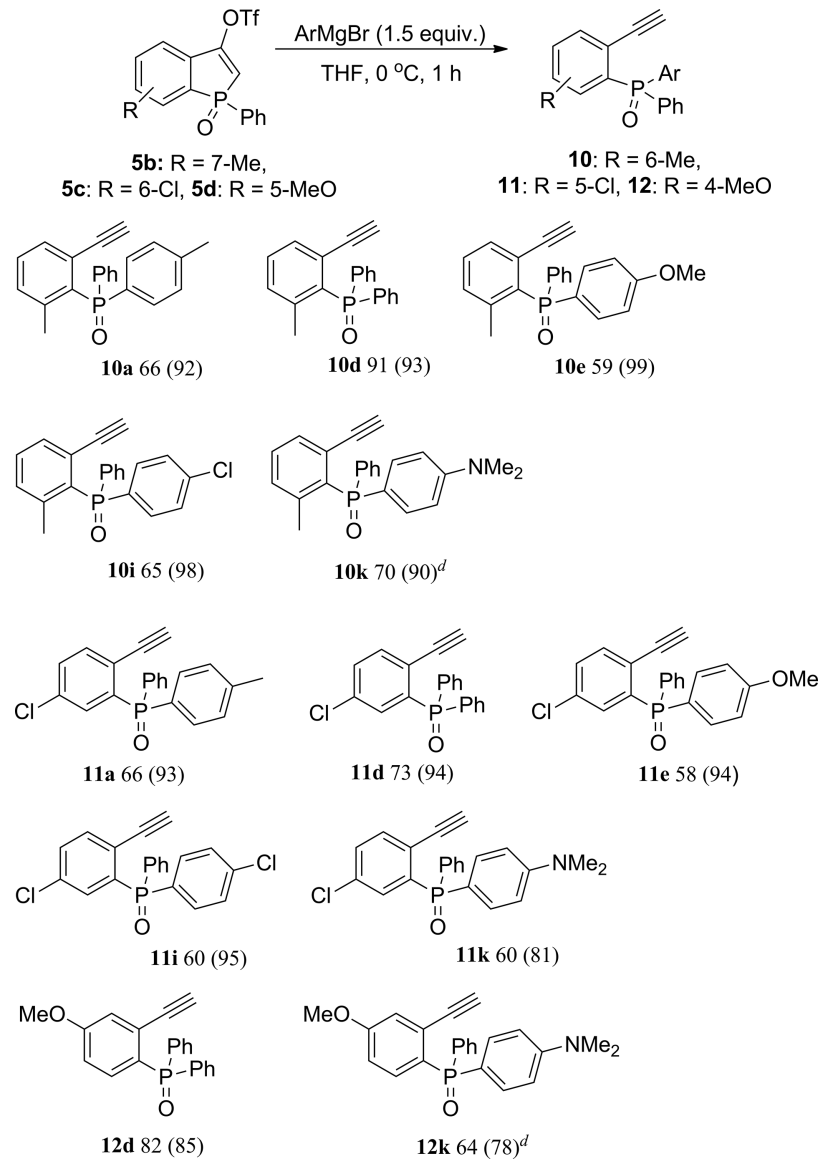

${ }^{a}$ Reaction conditions: $5 \mathbf{5 a}(0.13 \mathrm{mmol}), \operatorname{ArMgBr}(0.195 \mathrm{mmol})$, THF $(2 \mathrm{~mL}) .{ }^{b}$ Isolated yields of product. ${ }^{c}$ Numbers in parentheses indicate yields according to ${ }^{31} \mathrm{P}$ NMR. ${ }^{d}$ Formation of a small amount of ketone 3a was observed but not isolated.

out to be unreactive under the developed conditions, and $\mathbf{6 a}$ has been recovered in $72 \%$ yield (Scheme 4 ).

\section{Scheme 4. Reactivity of 6a toward $\mathrm{PhMgBr}$}

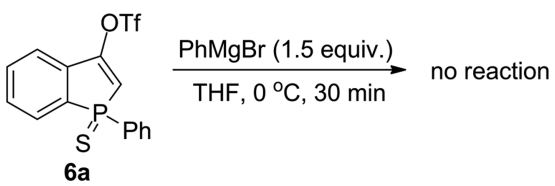

In the last part of the study, we subjected triflate 5a to the reactions with different organometallic nucleophiles (Scheme 5 ). In the case of the reaction with phenyllithium, the selectivity of the reaction toward the formation of $\mathbf{8 d}$ has lowered in comparison to the use of $\mathrm{PhMgBr}$. Compound $\mathbf{8 d}$ was still the main product but has remained in the mixture with other unidentified products. Finally, we were able to isolate $\mathbf{8 d}$ in a $21 \%$ yield. In turn, the reaction with the alkyl Grignard reagent has led to similar reactivity as it was observed for aryl Grignard reagents. As the result of the reaction of 5a with $\mathrm{EtMgBr}$, ethyl(2-ethynylphenyl)phenylphosphine oxide (13) was observed as a major product in the reaction mixture and isolated in $58 \%$ yield. 
Scheme 5. Reactivity of 5a toward Different Organometallic Nucleophiles
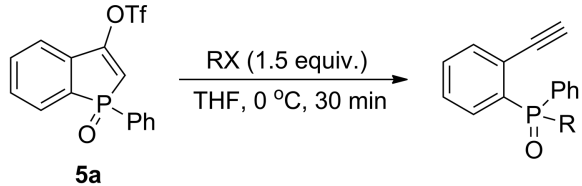

$\mathrm{RX}=\mathrm{PhLi} \quad \mathbf{8 d} 21(49)^{\mathrm{a}-\mathrm{c}}$ $\mathrm{RX}=\mathrm{EtMgBr} 1358(89)^{\mathrm{a}-\mathrm{c}}$

${ }^{a}$ Reaction conditions: $5 \mathrm{a}(0.108 \mathrm{mmol}), \mathrm{RX}(0.164 \mathrm{mmol}), \operatorname{THF}(2 \mathrm{~mL}){ }^{b}$ Isolated yields of product (\%). ${ }^{c}$ Numbers in parentheses indicate yields according to ${ }^{31} \mathrm{P}$ NMR (\%)

A plausible mechanism for the formation of 2ethynylphenyl(diaryl)phosphine oxides is depicted in Scheme 6 (eq 1). This includes the attack of an aryl Grignard reagent (II) on the phosphorus atom in triflate I, which, in turn, causes the opening of the five-membered ring and the formation of a triple bond in the ortho position to the phosphorus center. The consequence of the ring opening is the cleavage of the leaving group ( $\left.{ }^{-} \mathrm{OTf}\right)$, resulting in the formation of 2-ethynylphenyl(diaryl)phosphine oxide (III).

In eq 2 , a plausible mechanism of the subsequent reaction of phosphine oxide III, leading to compound $\mathbf{V}$, is presented. The deprotonation of the formed phosphine oxide III by an aryl Grignard reagent leads to the formation of an anion IV, which competes with the Grignard reagent in a reaction with another molecule of I. The attack of anion IV leads to an analogous ring opening of $\mathbf{I}$ as it was observed for the Grignard reagent. In accordance with eq 1 , the formation of a triple bond in the ortho position in compound $\mathbf{V}$ is observed. As a consequence, product $\mathbf{V}$ with an alkyne-phosphorus bond is formed.

\section{CONCLUSION}

In summary, we developed a facile method for the synthesis of 2-ethynylphenyl(diaryl)phosphine oxides from readily available reagents under mild conditions and in a short time. It is the first example of the preparation of arylphosphine oxides possessing a terminal ethynyl moiety in the ortho position in a phenyl ring. The mechanism for the formation of both 2ethynylphenyl(diaryl)phosphine oxides 8 and diphosphorus compound 9 has been proposed. The scope of the method for diverse substitution patterns in a structure of a Grignard reagent, benzophosphole core, and phosphorus atom has been investigated. The application of the method for the higherscale preparations has been confirmed. The study of reactivity of 2-ethynylphenyl(diaryl)phosphine oxides and compound 9 is underway in our laboratory.

\section{EXPERIMENTAL SECTION}

All reactions were performed under an argon atmosphere using Schlenk techniques. Only dry solvents were used, and glassware was heated under a vacuum prior use. All chemicals were used as received unless noted otherwise. Solvents for chromatography and crystallization were distilled once before use, and the solvents for extraction were used as received. THF and toluene were distilled from sodium/ benzophenone ketyl under argon. DCM was dried using $\mathrm{P}_{4} \mathrm{O}_{10}$ and distilled before use.

${ }^{1} \mathrm{H}$ NMR, ${ }^{31} \mathrm{P}\left\{{ }^{1} \mathrm{H}\right\}$ NMR, and ${ }^{13} \mathrm{C}\left\{{ }^{1} \mathrm{H}\right\}$ NMR spectra were recorded on a Bruker Advance 500 spectrometer at an ambient temperature in $\mathrm{CDCl}_{3}$ unless otherwise noted. Chemical shifts $(\delta)$ are reported in ppm from tetramethylsilane with the solvent as an internal indicator $\left(\mathrm{CDCl}_{3} 7.27 \mathrm{ppm}\right.$ for ${ }^{1} \mathrm{H}$ and $77 \mathrm{ppm}$ for $\left.{ }^{13} \mathrm{C}\right)$. Structural assignments were made with additional information from DEPT experiments. Mass spectra were recorded on Shimadzu GC-MS QP2010S in electron ionization (EI). Melting points were determined on a Büchi Melting Point M-560 in a capillary tube and were uncorrected. HPLC-HRMS was performed on a Shimazu HRMS ESI-IT-TOF using a reverse-phase stationary phase with water/ $\mathrm{MeCN}$ (65:35) as an eluent, electrospray ionization (ESI), and an ITTOF detector. Elementary analyses were performed on a Perkin Elmer CHN 2400. Thin-layer chromatography (TLC) was performed with precoated silica gel plates and visualized by UV light or $\mathrm{KMnO}_{4}$ solution or iodide on silica gel. The reaction mixtures were purified by column chromatography over silica gel (60-240 mesh).

Single crystals for 9 were obtained by dissolving $35 \mathrm{mg}$ of 9 (an oil from chromatography column) in about $1 \mathrm{~mL}$ of AcOEt. Then $24 \mathrm{~h}$ later at $25{ }^{\circ} \mathrm{C}$, colorless crystals appeared. Obtained crystals were isolated and dried for $24 \mathrm{~h}$ in rt.

The X-ray intensity data for 9 were collected on a diffractometer IPDS2T equipped with a STOE image plate detector system using a microfocus X-ray source providing $\mathrm{K} \alpha$ radiation by high-grade multilayer X-ray mirror optics for a Mo $(\lambda=0.71073 \AA)$ wavelength. The measurement was carried out at $120 \mathrm{~K}$. The structure of the compounds was solved by direct methods and refined against $F^{2}$ with the Shelxs-2008 and Shelxl-2008 programs ${ }^{33}$ run under WinGX. ${ }^{34}$ Non-hydrogen atoms were refined with anisotropic displacement parameters. The isotropic displacement parameters of all hydrogens were fixed to $1.2 U_{\text {eq }}$ for $\mathrm{CH}$ and $\mathrm{CH}_{2}$ (1.5 times for methyl groups).

Scheme 6. Plausible Mechanism of the Formation of Products 8a and 9

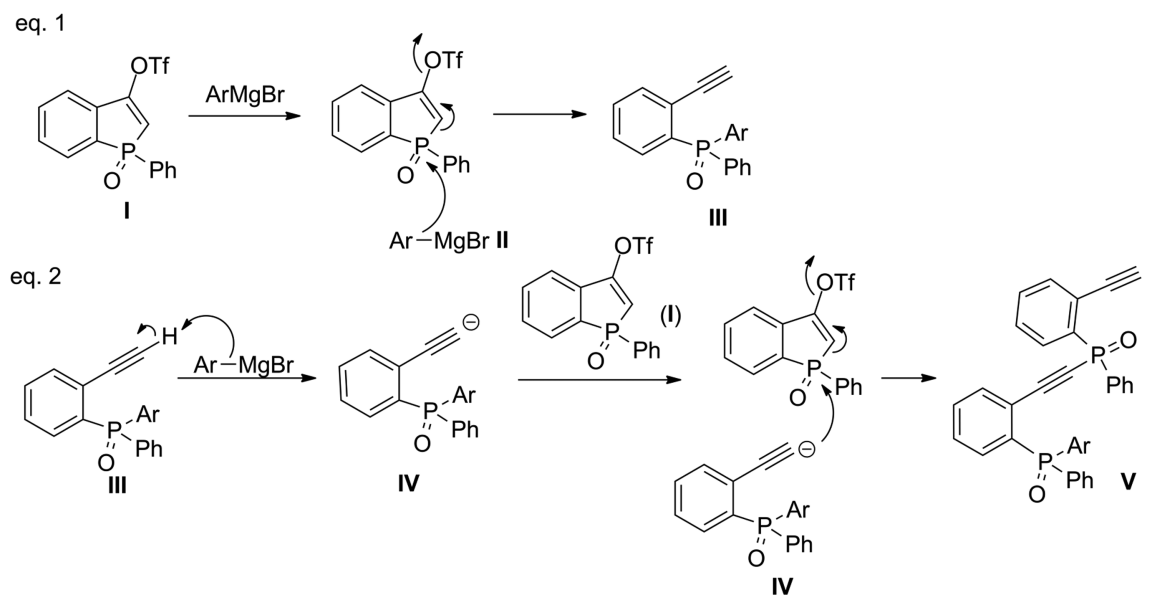


The crystallographic data for the structure of 9 reported in this work have been deposited in the Cambridge Crystallograic Data Centre (CCDC 2094907).

The starting compounds were prepared according to reported methods: phenyl(methyl)phosphine oxide, ${ }^{35}$ methyl 2-iodobenzoate, ${ }^{36}$ methyl 2-iodo-6-methylbenzoate, ${ }^{37}$ methyl 2-iodo-5-chlorobenzoate, ${ }^{38}$ 2-iodo-4-methoxybenzoic acid, ${ }^{39}$ methyl 2-iodo-4methoxybenzoate. ${ }^{40}$

Syntheses of 2-Methoxycarbonylphenylphosphine Oxides 2 Were Performed According to a Reported Procedure (General Procedure A). ${ }^{35}$ (2-Methoxycarbonylphenyl)(methyl)phenylphosphine Oxide (2a). ${ }^{35}$ Methyl(phenyl)phosphine oxide $(1.248 \mathrm{~g}, 8.91 \mathrm{mmol})$ was reacted according to a reported procedure ${ }^{35}$ with methyl 2-iodobenzoate $(2.567 \mathrm{~g}, 9.7 \mathrm{mmol})$ to afford $2 \mathrm{a}(66 \%$, $1.61 \mathrm{~g}, 5.9 \mathrm{mmol}) .{ }^{1} \mathrm{H} \mathrm{NMR}\left(500 \mathrm{MHz}, \mathrm{CDCl}_{3}\right): \delta 8.33-8.53(\mathrm{~m}$, $1 \mathrm{H}), 7.87-7.89(\mathrm{~m}, 1 \mathrm{H}), 7.68-7.71(\mathrm{~m}, 1 \mathrm{H}), 7.56-7.63(\mathrm{~m}, 3 \mathrm{H})$, $7.42-7.49(\mathrm{~m}, 1 \mathrm{H}), 7.38-7.42(\mathrm{~m}, 2 \mathrm{H}), 3.54(\mathrm{~s}, 3 \mathrm{H}), 2.22\left(\mathrm{~d}, J_{\mathrm{p}-\mathrm{H}}=\right.$ $13.87 \mathrm{~Hz}, 3 \mathrm{H}) \cdot{ }^{13} \mathrm{C}\left\{{ }^{1} \mathrm{H}\right\}$ NMR $\left(125 \mathrm{MHz}, \mathrm{CDCl}_{3}\right): \delta 167.0\left(\mathrm{~d}, J_{\mathrm{P}-\mathrm{H}}\right.$ $=2.7 \mathrm{~Hz}, \mathrm{C}), 134.5\left(\mathrm{~d}, J_{\mathrm{P}-\mathrm{H}}=107.2 \mathrm{~Hz}, \mathrm{C}\right), 134.3\left(\mathrm{~d}, J_{\mathrm{P}-\mathrm{H}}=7.3 \mathrm{~Hz}\right.$, $\mathrm{CH}), 133.8\left(\mathrm{~d}, J_{\mathrm{P}-\mathrm{H}}=92.6 \mathrm{~Hz}, \mathrm{C}\right), 133.6\left(\mathrm{~d}, J_{\mathrm{P}-\mathrm{H}}=6.4 \mathrm{~Hz}, \mathrm{CH}\right)$, $131.7(\mathrm{~s}), 131.6(\mathrm{~s}), 131.2\left(\mathrm{~d}, J_{\mathrm{P}-\mathrm{H}}=2.7 \mathrm{~Hz}, \mathrm{CH}\right), 130.2\left(\mathrm{~d}, J_{\mathrm{P}-\mathrm{H}}=\right.$ $8.2 \mathrm{~Hz}, \mathrm{CH}), 129.8\left(\mathrm{~d}, J_{\mathrm{P}-\mathrm{H}}=10.0 \mathrm{~Hz}, \mathrm{CH}\right), 128.2\left(\mathrm{~d}, J_{\mathrm{P}-\mathrm{H}}=11.8\right.$ $\mathrm{Hz}, \mathrm{CH}), 52.7\left(\mathrm{~s}, \mathrm{H}_{3} \mathrm{CO}\right), 16.3\left(\mathrm{~d}, J_{\mathrm{P}-\mathrm{H}}=77.2 \mathrm{~Hz}, \mathrm{CH}_{3}\right) .{ }^{31} \mathrm{P}\left\{{ }^{1} \mathrm{H}\right\}$ NMR $\left(202 \mathrm{MHz}, \mathrm{CDCl}_{3}\right): \delta 33.45$ (s).

[(2-Methoxycarbonyl)-6-methylphenyl]methylphenylphosphine Oxide (2b). Methyl(phenyl)phosphine oxide (2.2 g, $15.7 \mathrm{mmol}$ ) was reacted according to a reported procedure ${ }^{35}$ with methyl 2-iodo-6methylbenzoate $(4.77 \mathrm{~g}, 17.4 \mathrm{mmol})$ to afford $2 \mathbf{b}$ as a brown solid $(65 \%, 2.96 \mathrm{~g}, 10.2 \mathrm{mmol}) . \mathrm{Mp}: 73.4-74.2{ }^{\circ} \mathrm{C} . R_{f}=0.34$ (30:5:1 $\left.\mathrm{CHCl}_{3} / \mathrm{AcOEt} / \mathrm{MeOH}\right) .{ }^{1} \mathrm{H}$ NMR $\left(500 \mathrm{MHz}, \mathrm{CDCl}_{3}\right): \delta 7.66-7.70$ $(\mathrm{m}, 2 \mathrm{H}), 7.49-7.53(\mathrm{~m}, 1 \mathrm{H}), 7.43-7.56(\mathrm{~m}, 3 \mathrm{H}), 7.36-7.40(\mathrm{~m}$, $1 \mathrm{H}), 7.27-7.31(\mathrm{~m}, 1 \mathrm{H}), 3.69(\mathrm{~s}, 3 \mathrm{H}), 2.35(\mathrm{~s}, 3 \mathrm{H}), 2.17\left(\mathrm{~d}, J_{\mathrm{P}-\mathrm{H}}=\right.$ $13.40 \mathrm{~Hz}, 3 \mathrm{H}) .{ }^{13} \mathrm{C}\left\{{ }^{1} \mathrm{H}\right\} \mathrm{NMR}\left(125 \mathrm{MHz}, \mathrm{CDCl}_{3}\right): \delta 170.2\left(\mathrm{~d}, J_{\mathrm{C}-\mathrm{P}}=\right.$ $4.5 \mathrm{~Hz}, \mathrm{C}), 142.6$ (d, $\left.J_{\mathrm{C}-\mathrm{P}}=9.1 \mathrm{~Hz}, \mathrm{C}\right), 139.1\left(\mathrm{~d}, J_{\mathrm{C}-\mathrm{P}}=7.3 \mathrm{~Hz}, \mathrm{C}\right)$, $135.1\left(\mathrm{~d}, J_{\mathrm{C}-\mathrm{P}}=103.5 \mathrm{~Hz}, \mathrm{C}\right), 133.6\left(\mathrm{~d}, J_{\mathrm{P}-\mathrm{C}}=10.0 \mathrm{~Hz}, \mathrm{CH}\right), 131.6$ $\left(\mathrm{d}, J_{\mathrm{C}-\mathrm{P}}=2.7 \mathrm{~Hz}, \mathrm{CH}\right), 131.2\left(\mathrm{~d}, J_{\mathrm{C}-\mathrm{P}}=1.8 \mathrm{~Hz}, \mathrm{CH}\right), 130.1\left(\mathrm{~d}, J_{\mathrm{C}-\mathrm{P}}\right.$ $=10.9 \mathrm{~Hz}, 2 \mathrm{CH}), 129.9\left(\mathrm{~d}, J_{\mathrm{C}-\mathrm{P}}=94.5 \mathrm{~Hz}, \mathrm{C}\right), 128.6\left(\mathrm{~d}, J_{\mathrm{C}-\mathrm{P}}=11.8\right.$ $\mathrm{Hz}, 2 \mathrm{CH}), 126.1\left(\mathrm{~d}, J_{\mathrm{C}-\mathrm{P}}=9.1 \mathrm{~Hz}, \mathrm{CH}\right), 52.6\left(\mathrm{~s}, \mathrm{H}_{3} \mathrm{CO}\right), 22.7(\mathrm{~d}$, $\left.J_{\mathrm{C}-\mathrm{P}}=4.5 \mathrm{~Hz}, \mathrm{CH}_{3}\right), 16.6\left(\mathrm{~d}, J_{\mathrm{C}-\mathrm{P}}=74.6 \mathrm{~Hz}, \mathrm{CH}_{3}\right) .{ }^{31} \mathrm{P}\left\{{ }^{1} \mathrm{H}\right\} \mathrm{NMR}$ $\left(202 \mathrm{MHz}, \mathrm{CDCl}_{3}\right): \delta 32.57$ (s). GC-MS (EI) $\mathrm{m} / z: 274$ (29) (M$\left.\mathrm{CH}_{3}+\mathrm{H}\right)^{+}, 273(100)\left(\mathrm{M}-\mathrm{CH}_{3}\right)^{+}, 256(14), 213(10), 195$ (27), 179 (8), 178 (8), 167 (13), 166 (15), 165 (48), 152 (16), 139 (24), 137 (11), 121 (12), 109 (12), 107 (9), 91 (29), 90 (18), 89 (46), 79 (14), 78 (60), 77 (99), 74 (16). HRMS (ESI/Q-TOF) $\mathrm{m} / z:[\mathrm{M}+$ $\mathrm{Na}]^{+}$calcd for $\mathrm{C}_{16} \mathrm{H}_{17} \mathrm{O}_{3} \mathrm{PNa}, 311.0808$; found, 311.0802 .

[(2-Methoxycarbonyl)-5-chlorophenyl]methylphenylphosphine Oxide (2c). Methyl(phenyl)phosphine oxide (2.41 g, $17.2 \mathrm{mmol}$ ) was reacted according to a reported procedure ${ }^{35}$ with methyl 5-chloro-2iodo-benzoate $(5.16 \mathrm{~g}, 17.4 \mathrm{mmol})$ to afford $2 \mathrm{c}$ as a brown solid $(52 \%, 2.74 \mathrm{~g}, 8.9 \mathrm{mmol}) . \mathrm{Mp}: 111-112{ }^{\circ} \mathrm{C} . R_{f}=0.38\left(30: 5: 1 \mathrm{CHCl}_{3} /\right.$ AcOEt/MeOH). ${ }^{1} \mathrm{H}$ NMR $\left(500 \mathrm{MHz}, \mathrm{CDCl}_{3}\right): \delta 8.43-8.46(\mathrm{~m}$, $1 \mathrm{H}), 7.85-7.88(\mathrm{~m}, 1 \mathrm{H}), 7.58-7.63(\mathrm{~m}, 3 \mathrm{H}), 7.47-7.51(\mathrm{~m}, 1 \mathrm{H})$, $7.41-7.45(\mathrm{~m}, 2 \mathrm{H}), 3.56(\mathrm{~s}, 3 \mathrm{H}), 2.25\left(\mathrm{~d}, J_{\mathrm{P}-\mathrm{H}}=14.19 \mathrm{~Hz}, 3 \mathrm{H}\right)$. ${ }^{13} \mathrm{C}\left\{{ }^{1} \mathrm{H}\right\} \operatorname{NMR}\left(125 \mathrm{MHz}, \mathrm{CDCl}_{3}\right): \delta 166.1\left(\mathrm{~d}, J_{\mathrm{C}-\mathrm{P}}=1.8 \mathrm{~Hz}, \mathrm{C}\right)$, $139.0\left(\mathrm{~d}, J_{\mathrm{C}-\mathrm{P}}=13.6 \mathrm{~Hz}, \mathrm{C}\right), 136.7\left(\mathrm{~d}, J_{\mathrm{C}-\mathrm{P}}=89.0 \mathrm{~Hz}, \mathrm{C}\right), 134.7$ (d, $\left.J_{\mathrm{C}-\mathrm{P}}=7.3 \mathrm{~Hz}, \mathrm{CH}\right), 134.1\left(\mathrm{~d}, J_{\mathrm{C}-\mathrm{P}}=108.1 \mathrm{~Hz}, \mathrm{C}\right), 131.8\left(\mathrm{~d}, J_{\mathrm{C}-\mathrm{P}}=\right.$ $9.1 \mathrm{~Hz}, \mathrm{CH}), 131.7\left(\mathrm{~d}, J_{\mathrm{C}-\mathrm{P}}=1.8 \mathrm{~Hz}, \mathrm{CH}\right), 131.5\left(\mathrm{~d}, J_{\mathrm{C}-\mathrm{P}}=2.7 \mathrm{~Hz}\right.$, $\mathrm{CH}), 129.9\left(\mathrm{~d}, J_{\mathrm{C}-\mathrm{P}}=10.0 \mathrm{~Hz}, \mathrm{CH}\right), 128.3\left(\mathrm{~d}, J_{\mathrm{C}-\mathrm{p}}=12.7 \mathrm{~Hz}, \mathrm{CH}\right)$, $52.4\left(\mathrm{~s}, \mathrm{CH}_{3}\right), 16.0\left(\mathrm{~d}, J_{\mathrm{C}-\mathrm{P}}=78.1 \mathrm{~Hz}, \mathrm{CH}_{3}\right) \cdot{ }^{31} \mathrm{P}\left\{{ }^{1} \mathrm{H}\right\}$ NMR $(202$ $\mathrm{MHz}, \mathrm{CDCl}_{3}$ ): $\delta 32.44$ (s). GC-MS (EI) $\mathrm{m} / z: 295$ (22), 294 (7),

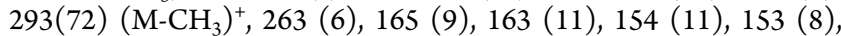
152 (18), 151 (17), 139 (28), 138 (17), 126 (10). HRMS (ESI/QTOF) $m / z:[\mathrm{M}+\mathrm{Na}]^{+}$calcd for $\mathrm{C}_{15} \mathrm{H}_{14} \mathrm{ClO}_{3} \mathrm{PNa}, 331.0261$; found, 331.0270 .

[(2-Methoxycarbonyl)-4-methoxyphenyl]methylphenylphosphine Oxide (2d). Methyl(phenyl)phosphine oxide $(2.27 \mathrm{~g}, 16.2 \mathrm{mmol})$ was reacted according to a reported procedure $^{35}$ with methyl 2-iodo-4-methoxy-benzoate (5.25 g, 17.98 $\mathrm{mmol})$ to afford $2 \mathrm{~d}$ as a yellowish solid $(55 \%, 2.71 \mathrm{~g}, 8.91 \mathrm{mmol})$. Mp: $115.9-116.5{ }^{\circ} \mathrm{C} . R_{f}=0.28\left(30: 5: 1 \mathrm{CHCl}_{3} / \mathrm{AcOEt} / \mathrm{MeOH}\right) .{ }^{1} \mathrm{H}$
NMR (500 MHz, $\left.\mathrm{CDCl}_{3}\right): \delta 8.26-8.31(\mathrm{~m}, 1 \mathrm{H}), 7.58-7.62(\mathrm{~m}, 2 \mathrm{H})$ 7.42-7.49 (m, 1H), 7.39-7.41 (m, 3H), 7.17-7.20 (m, 1H), $3.89(\mathrm{~s}$, $3 \mathrm{H}), 3.54(\mathrm{~s}, 3 \mathrm{H}), 2.19\left(\mathrm{~d}, J_{\mathrm{P}-\mathrm{H}}=14.2 \mathrm{~Hz}, 3 \mathrm{H}\right) .{ }^{13} \mathrm{C}\left\{{ }^{1} \mathrm{H}\right\}$ NMR $(125$ $\left.\mathrm{MHz}, \mathrm{CDCl}_{3}\right): \delta 166.8\left(\mathrm{~d}, J_{\mathrm{C}-\mathrm{P}}=1.8 \mathrm{~Hz}, \mathrm{C}\right), 162.0\left(\mathrm{~d}, J_{\mathrm{C}-\mathrm{P}}=1.8 \mathrm{~Hz}\right.$, C), $136.4\left(\mathrm{~d}, J_{\mathrm{C}-\mathrm{P}}=9.1 \mathrm{~Hz}, \mathrm{CH}\right), 135.3\left(\mathrm{~d}, J_{\mathrm{C}-\mathrm{P}}=7.3 \mathrm{~Hz}, \mathrm{C}\right), 135.1$ $\left(\mathrm{d}, J_{\mathrm{C}-\mathrm{P}}=106.3 \mathrm{~Hz}, \mathrm{C}\right), 131.1\left(\mathrm{~d}, J_{\mathrm{C}-\mathrm{P}}=2.7 \mathrm{~Hz}, \mathrm{CH}\right), 129.8\left(\mathrm{~d}, J_{\mathrm{C}-\mathrm{P}}\right.$ $=10.0 \mathrm{~Hz}, 2 \mathrm{CH}), 128.2\left(\mathrm{~d}, J_{\mathrm{C}-\mathrm{P}}=12.7 \mathrm{~Hz}, 2 \mathrm{CH}\right), 124.8\left(\mathrm{~d}, J_{\mathrm{C}-\mathrm{P}}=\right.$ $100.0 \mathrm{~Hz}, \mathrm{C}), 116.5\left(\mathrm{~d}, J_{\mathrm{C}-\mathrm{P}}=2.7 \mathrm{~Hz}, \mathrm{CH}\right), 116.4\left(\mathrm{~d}, J_{\mathrm{C}-\mathrm{P}}=5.5 \mathrm{~Hz}\right.$, $\mathrm{CH}), 55.6\left(\mathrm{~s}, \mathrm{CH}_{3}\right), 52.2\left(\mathrm{~s}, \mathrm{CH}_{3}\right), 16.6\left(\mathrm{~d}, J_{\mathrm{C}-\mathrm{P}}=78.1 \mathrm{~Hz}, \mathrm{CH}_{3}\right)$. ${ }^{31} \mathrm{P}\left\{{ }^{1} \mathrm{H}\right\}$ NMR $\left(202 \mathrm{MHz}, \mathrm{CDCl}_{3}\right): \delta 32.55(\mathrm{~s}) . \mathrm{GC}-\mathrm{MS}(\mathrm{EI}): \mathrm{m} / z$

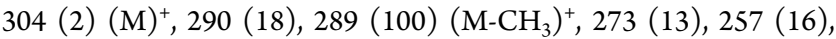
227 (13), 211 (13), 139 (31), 121 (11), 107 (10), 91 (14), 77 (50). HRMS (ESI/Q-TOF) $m / z:[\mathrm{M}+\mathrm{H}]^{+}$calcd for $\mathrm{C}_{16} \mathrm{H}_{17} \mathrm{O}_{4} \mathrm{P}$, 305.0937; found, 305.0929 .

Synthesis of Benzophospholan-3-one Oxides 3 (General Procedure B). ${ }^{41}$ To a Schlenk tube $(100 \mathrm{~mL})$ equipped with a magnetic stirrer and an argon inlet phosphine was added oxide 2 (2.0 $\mathrm{mmol}$ ) in anhydrous THF $(15 \mathrm{~mL})$, and the mixture was cooled to $-78{ }^{\circ} \mathrm{C}$ with a dry ice/acetone bath. Then, LDA $(2.5 \mathrm{~mL}, 5.0 \mathrm{mmol}$, $2 \mathrm{M}$ solution) was added, and the mixture was stirred at the same temperature for $30 \mathrm{~min}$. After that time, the ice bath was removed. The reaction mixture was stirred at rt overnight and then quenched by the addition of a saturated $\mathrm{NH}_{4} \mathrm{Cl}$ solution $(5 \mathrm{~mL})$ and extracted with $\mathrm{CHCl}_{3}(5 \times 10 \mathrm{~mL})$. The collected organic phases were dried over $\mathrm{Na}_{2} \mathrm{SO}_{4}$, the solid was filtered off, and the filtrate was evaporated under reduced pressure. The crude residue was checked using NMR techniques. Then, the crude mixture was dissolved in DCM $(30 \mathrm{~mL})$, solution of $\mathrm{HCl}(1 \mathrm{M}, 10 \mathrm{~mL})$ was added, and the mixture was vigorously stirred for $30 \mathrm{~min}$. Then the acidic water phase was removed in a separating funnel, the organic phase was returned to the flask, and another portion of $\mathrm{HCl}(1 \mathrm{M}, 10 \mathrm{~mL})$ was added. The mixture was once again vigorously stirred for $30 \mathrm{~min}$, and after that time, the acidic water phase was separated. The organic phase was washed with water $(10 \mathrm{~mL})$ and dried over $\mathrm{Na}_{2} \mathrm{SO}_{4}$. The solid was filtered off, and the filtrate was evaporated under reduced pressure. The residue was purified by short $(5 \mathrm{~cm})$ column chromatography on silica gel using $\mathrm{CHCl}_{3} /$ acetone $(2: 1 \mathrm{v} / \mathrm{v})$ or $\mathrm{CHCl}_{3} / \mathrm{EtOAc} / \mathrm{MeOH}$ $(30: 5: 1 \mathrm{v} / \mathrm{v} / \mathrm{v})$ as an eluent.

Benzophospholan-3-one Oxide (3a). ${ }^{29}$ Compound 2a (0.55 g, 2.0 $\mathrm{mmol}$ ) was reacted according to general procedure $\mathrm{B}$ to afford $3 \mathrm{a}$ $(55 \%, 0.266 \mathrm{~g}, 1.1 \mathrm{mmol}) .{ }^{1} \mathrm{H}$ NMR $\left(500 \mathrm{MHz}, \mathrm{CDCl}_{3}\right): \delta 8.03-8.07$ (m, $1 \mathrm{H}), 7.85-7.90(\mathrm{~m}, 1 \mathrm{H}), 7.75-7.83(\mathrm{~m}, 2 \mathrm{H}), 7.52-7.60(\mathrm{~m}$, $3 \mathrm{H}), 7.44-7.48(\mathrm{~m}, 2 \mathrm{H}), 3.12-3.31(\mathrm{~m}, 2 \mathrm{H}) .{ }^{13} \mathrm{C}\left\{{ }^{1} \mathrm{H}\right\}$ NMR $(125$ $\left.\mathrm{MHz} \mathrm{CDCl}_{3}\right): \delta 194.3\left(\mathrm{~d}, J_{\mathrm{C}-\mathrm{P}}=12.7 \mathrm{~Hz}, \mathrm{C}\right), 141.5\left(\mathrm{~d}, J_{\mathrm{C}-\mathrm{P}}=91.7\right.$ $\mathrm{Hz}, \mathrm{C}), 141.3\left(\mathrm{~d}, J_{\mathrm{C}-\mathrm{P}}=12.7 \mathrm{~Hz}, \mathrm{C}\right), 135.9$ (d, $\left.J_{\mathrm{C}-\mathrm{P}}=10.9 \mathrm{~Hz}, \mathrm{CH}\right)$, $133.6\left(\mathrm{~d}, J_{\mathrm{C}-\mathrm{p}}=2.7 \mathrm{~Hz}, \mathrm{CH}\right), 132.5\left(\mathrm{~d}, J_{\mathrm{C}-\mathrm{P}}=2.7 \mathrm{~Hz}, \mathrm{CH}\right), 130.8(\mathrm{~d}$, $\left.J_{\mathrm{C}-\mathrm{P}}=105.4 \mathrm{~Hz}, \mathrm{C}\right), 130.6\left(\mathrm{~d}, J_{\mathrm{C}-\mathrm{P}}=10.9 \mathrm{~Hz}, 2 \mathrm{CH}\right), 129.3\left(\mathrm{~d}, J_{\mathrm{C}-\mathrm{P}}=\right.$ $6.4 \mathrm{~Hz}, \mathrm{CH}), 128.9\left(\mathrm{~d}, J_{\mathrm{C}-\mathrm{P}}=13.6 \mathrm{~Hz}, \mathrm{CH}\right), 124.6\left(\mathrm{~d}, J_{\mathrm{C}-\mathrm{P}}=10.9\right.$ $\mathrm{Hz}, \mathrm{CH}), 40.1\left(\mathrm{~d}, J_{\mathrm{C}-\mathrm{P}}=71.8 \mathrm{~Hz}, \mathrm{CH}_{2}\right) .{ }^{31} \mathrm{P}\left\{{ }^{1} \mathrm{H}\right\} \mathrm{NMR}(202 \mathrm{MHz}$, $\left.\mathrm{CDCl}_{3}\right): \delta 29.92(\mathrm{~s})$.

7-Methylbenzophospholan-3-one Oxide (3b). Compound $\mathbf{2 b}$ $(0.577 \mathrm{~g}, 2.0 \mathrm{mmol})$ was reacted according to general procedure $\mathrm{B}$ to afford $3 \mathbf{b}$ as a beige solid $(55 \%, 0.28 \mathrm{~g}, 1.1 \mathrm{mmol})$. Mp: $165-166$ ${ }^{\circ} \mathrm{C}$ (dec.). $R_{f}=0.66$ (2:1 $\mathrm{CHCl}_{3}$ /acetone). ${ }^{1} \mathrm{H}$ NMR $(500 \mathrm{MHz}$, $\left.\mathrm{CDCl}_{3}\right): \delta 7.78-7.7 .92(\mathrm{~m}, 1 \mathrm{H}), 7.64-7.68(\mathrm{~m}, 1 \mathrm{H}), 7.53-7.63(\mathrm{~m}$ $4 \mathrm{H}), 7.46-7.58(\mathrm{~m}, 2 \mathrm{H}), 3.09-3.32(\mathrm{~m}, 2 \mathrm{H}), 2.43(\mathrm{~s}, 3 \mathrm{H}) .{ }^{13} \mathrm{C}\left\{{ }^{1} \mathrm{H}\right\}$

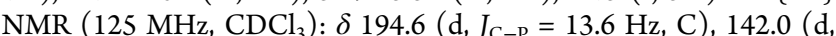
$\left.J_{\mathrm{C}-\mathrm{P}}=5.5 \mathrm{~Hz}, \mathrm{C}\right), 141.8\left(\mathrm{~d}, J_{\mathrm{C}-\mathrm{P}}=12.7 \mathrm{~Hz}, \mathrm{C}\right), 139.7\left(\mathrm{~d}, J_{\mathrm{C}-\mathrm{P}}=90.8\right.$ $\mathrm{Hz}, \mathrm{C}), 137.1\left(\mathrm{~d}, J_{\mathrm{C}-\mathrm{P}}=9.1 \mathrm{~Hz}, \mathrm{CH}\right), 133.9\left(\mathrm{~d}, J_{\mathrm{C}-\mathrm{P}}=1.8 \mathrm{~Hz}, \mathrm{CH}\right)$, $132.4\left(\mathrm{~d}, J_{\mathrm{C}-\mathrm{P}}=3.6 \mathrm{~Hz}, \mathrm{CH}\right), 130.9\left(\mathrm{~d}, J_{\mathrm{C}-\mathrm{P}}=103.5 \mathrm{~Hz}, \mathrm{C}\right), 130.6(\mathrm{~d}$, $\left.J_{\mathrm{C}-\mathrm{P}}=10.9 \mathrm{~Hz}, \mathrm{CH}\right), 129.0\left(\mathrm{~d}, J_{\mathrm{C}-\mathrm{P}}=12.7 \mathrm{~Hz}, \mathrm{CH}\right), 122.2\left(\mathrm{~d}, J_{\mathrm{C}-\mathrm{P}}=\right.$ $11.8 \mathrm{~Hz}, \mathrm{CH}), 40.5\left(\mathrm{~d}, J_{\mathrm{C}-\mathrm{P}}=71.5 \mathrm{~Hz}, \mathrm{CH}_{2}\right), 19.4\left(\mathrm{~d}, J_{\mathrm{C}-\mathrm{P}}=4.5 \mathrm{~Hz}\right.$, $\left.\mathrm{CH}_{3}\right) \cdot{ }^{31} \mathrm{P}\left\{{ }^{1} \mathrm{H}\right\}$ NMR $\left(202 \mathrm{MHz}, \mathrm{CDCl}_{3}\right): \delta 30.36(\mathrm{~s})$. GC-MS (EI) $m / z: 258(7), 257(13), 256(63)(\mathrm{M})^{+}, 255(37), 236(8), 213(24)$, 179 (9), 166 (28), 165 (42), 151 (10), 150 (12), 137 (14), 121 (12), 106 (29). HRMS (ESI/Q-TOF) $\mathrm{m} / z$ : calcd for $\mathrm{C}_{15} \mathrm{H}_{13} \mathrm{O}_{2} \mathrm{P}[\mathrm{M}+$ $\mathrm{Na}]^{+}, 279.0545$; found, 279.0553 .

6-Chlorobenzophospholan-3-one Oxide (3c). Compound 2c $(0.617 \mathrm{~g}, 2.0 \mathrm{mmol})$ was reacted according to general procedure $\mathrm{B}$ to afford $3 \mathrm{c}$ as an orange oil $(55 \%, 0.3 \mathrm{~g}, 1.1 \mathrm{mmol}) . R_{f}=0.36(30: 5: 1$ 
$\mathrm{CHCl}_{3} / \mathrm{AcOEt} / \mathrm{MeOH}$ ). ${ }^{1} \mathrm{H} \mathrm{NMR}\left(500 \mathrm{MHz}, \mathrm{CDCl}_{3}\right.$ ): $\delta$ 7.99-8.01 $(\mathrm{m}, 1 \mathrm{H}), 7.81-7.84(\mathrm{~m}, 1 \mathrm{H}), 7.72-7.74(\mathrm{~m}, 1 \mathrm{H}), 7.58-7.62(\mathrm{~m}$, $3 \mathrm{H}), 7.49-7.52(\mathrm{~m}, 2 \mathrm{H}), 3.16-3.36(\mathrm{~m}, 2 \mathrm{H}) .{ }^{13} \mathrm{C}\left\{{ }^{1} \mathrm{H}\right\}$ NMR $(125$ $\left.\mathrm{MHz}, \mathrm{CDCl}_{3}\right): \delta 192.9\left(\mathrm{~d}, J_{\mathrm{C}-\mathrm{P}}=12.7 \mathrm{~Hz}, \mathrm{C}\right), 143.5\left(\mathrm{~d}, J_{\mathrm{C}-\mathrm{P}}=108.1\right.$ $\mathrm{Hz}, \mathrm{C}$ ), 143.1 (d, $\left.J_{\mathrm{C}-\mathrm{P}}=33.6 \mathrm{~Hz}, \mathrm{C}\right), 139.5$ (d, $J_{\mathrm{C}-\mathrm{P}}=12.7 \mathrm{~Hz}, \mathrm{C}$ ), $134.2\left(\mathrm{~d}, J_{\mathrm{C}-\mathrm{P}}=2.7 \mathrm{~Hz}, \mathrm{CH}\right), 132.9\left(\mathrm{~d}, J_{\mathrm{C}-\mathrm{P}}=3.6 \mathrm{~Hz}, \mathrm{CH}\right), 130.7(\mathrm{~d}$, $\left.J_{\mathrm{C}-\mathrm{P}}=10.9 \mathrm{~Hz}, \mathrm{CH}\right), 130.1\left(\mathrm{~d}, J_{\mathrm{C}-\mathrm{P}}=106.3 \mathrm{~Hz}, \mathrm{C}\right), 129.3\left(\mathrm{~d}, J_{\mathrm{C}-\mathrm{P}}=\right.$ $6.4 \mathrm{~Hz}, \mathrm{CH}), 129.2\left(\mathrm{~d}, J_{\mathrm{C}-\mathrm{P}}=14.5 \mathrm{~Hz}, \mathrm{CH}\right), 126.1\left(\mathrm{~d}, J_{\mathrm{C}-\mathrm{P}}=11.8\right.$ $\mathrm{Hz}, \mathrm{CH}), 40.2\left(\mathrm{~d}, J_{\mathrm{C}-\mathrm{P}}=71.8 \mathrm{~Hz}, \mathrm{CH}_{2}\right) .{ }^{31} \mathrm{P}\left\{{ }^{1} \mathrm{H}\right\} \mathrm{NMR}(202 \mathrm{MHz}$, $\mathrm{CDCl}_{3}$ ): $\delta 32.55$ (s). GC-MS (EI) $\mathrm{m} / z: 278$ (14), 277 (7), 276 (49) $(\mathrm{M})^{+}, 235$ (14), 234 (14), 233 (57). HRMS (ESI/Q-TOF) $\mathrm{m} / z$ : calcd for $\mathrm{C}_{14} \mathrm{H}_{10} \mathrm{ClO}_{2} \mathrm{P}[\mathrm{M}+\mathrm{H}]^{+}, 277.0180$; found, 277.0180 .

5-Methoxybenzophospholan-3-one Oxide (3d). Compound $2 \mathrm{~d}$ $(0.609 \mathrm{~g}, 2.0 \mathrm{mmol})$ was reacted according to general procedure $\mathrm{B}$ to afford $3 \mathrm{~d}$ as a brown oil $(59 \%, 0.32 \mathrm{~g}, 1.18 \mathrm{mmol}) . R_{f}=0.72(2: 1$ $\mathrm{CHCl}_{3}$ /acetone). ${ }^{1} \mathrm{H}$ NMR (500 $\left.\mathrm{MHz}, \mathrm{CDCl}_{3}\right): \delta 7.74-7.78(\mathrm{~m}$, $1 \mathrm{H}), 7.52-7.59(\mathrm{~m}, 3 \mathrm{H}), 7.44-7.47(\mathrm{~m}, 3 \mathrm{H}), 7.32-7.35(\mathrm{~m}, 1 \mathrm{H})$, $3.93(\mathrm{~m}, 3 \mathrm{H}), 3.11-3.32(\mathrm{~m}, 2 \mathrm{H}) .{ }^{13} \mathrm{C}\left\{{ }^{1} \mathrm{H}\right\}$ NMR $(125 \mathrm{MHz}$, $\left.\mathrm{CDCl}_{3}\right): \delta 194.3\left(\mathrm{~d}, J_{\mathrm{C}-\mathrm{P}}=12.7 \mathrm{~Hz}, \mathrm{C}\right), 164.2\left(\mathrm{~d}, J_{\mathrm{C}-\mathrm{P}}=2.7 \mathrm{~Hz}, \mathrm{C}\right)$, $144.0\left(\mathrm{~d}, J_{\mathrm{C}-\mathrm{P}}=14.5 \mathrm{~Hz}, \mathrm{C}\right), 133.2\left(\mathrm{~d}, J_{\mathrm{C}-\mathrm{P}}=97.2 \mathrm{~Hz}, \mathrm{C}\right), 132.4(\mathrm{~d}$, $\left.J_{\mathrm{C}-\mathrm{P}}=3.6 \mathrm{~Hz}, \mathrm{CH}\right), 133.2\left(\mathrm{~d}, J_{\mathrm{C}-\mathrm{P}}=107.2 \mathrm{~Hz}, \mathrm{C}\right), 130.7\left(\mathrm{~d}, J_{\mathrm{C}-\mathrm{P}}=\right.$ $10.7 \mathrm{~Hz}, 2 \mathrm{CH}), 130.6\left(\mathrm{~d}, J_{\mathrm{C}-\mathrm{P}}=7.4 \mathrm{~Hz}, \mathrm{CH}\right), 129.0\left(\mathrm{~d}, J_{\mathrm{C}-\mathrm{P}}=12.9\right.$ $\mathrm{Hz}, 2 \mathrm{CH}), 124.7\left(\mathrm{~d}, J_{\mathrm{C}-\mathrm{P}}=11.7 \mathrm{~Hz}, \mathrm{CH}\right), 106.5\left(\mathrm{~d}, J_{\mathrm{C}-\mathrm{P}}=12.6 \mathrm{~Hz}\right.$, $\mathrm{CH}), 55.9\left(\mathrm{~s}, \mathrm{OCH}_{3}\right), 40.8\left(\mathrm{~d}, J_{\mathrm{C}-\mathrm{P}}=71.8 \mathrm{~Hz}, \mathrm{CH}_{2}\right) .{ }^{31} \mathrm{P}\left\{{ }^{1} \mathrm{H}\right\} \mathrm{NMR}$ $\left(202 \mathrm{MHz}, \mathrm{CDCl}_{3}\right.$ ): $\delta 28.92$ (s). GC-MS (EI) $\mathrm{m} / z: 273$ (24), 272 (100) $(\mathrm{M})^{+}, 271$ (17), 256 (12), 244 (21), 229 (49), 210 (12), 208 (15), 195 (18), 193 (19), 183 (10), 179 (31), 165 (21), 153 (12), 152 (29), 148 (13), 139 (21), 137 (11), 120 (43), 107 (24), 91 (36), 89 (14). HRMS (ESI/Q-TOF) $m / z$ : calcd for $\mathrm{C}_{15} \mathrm{H}_{13} \mathrm{O}_{3} \mathrm{P}[\mathrm{M}+\mathrm{H}]^{+}$, 273.0675; found, 273.0681 .

Synthesis of Benzophospholan-3-one Sulfide (4a, General Procedure C). To a Schlenk tube $(100 \mathrm{~mL})$ equipped with a magnetic stirrer and an argon inlet was added phosphine oxide 3a $(0.528 \mathrm{~g}, 2.18 \mathrm{mmol})$ in anhydrous toluene $(20 \mathrm{~mL})$. Then, $\mathrm{HSiCl}_{3}$ $(2.2 \mathrm{~mL}, 21.8 \mathrm{mmol})$ was added, and the mixture was stirred at $\mathrm{rt}$ for $18 \mathrm{~h}$. The crude reaction mixture was checked using the ${ }^{31} \mathrm{P}\left\{{ }^{1} \mathrm{H}\right\}$ NMR technique. Then, sulfur $(21.8 \mathrm{mmol})$ was added, and the reaction mixture was stirred at $\mathrm{rt}$ for $8 \mathrm{~h}$. Then, the reaction mixture was quenched by the addition of saturated $\mathrm{NH}_{4} \mathrm{Cl}$ solution $(5 \mathrm{~mL})$ and extracted with $\mathrm{CHCl}_{3}(5 \times 10 \mathrm{~mL})$. The collected organic phases were dried over $\mathrm{Na}_{2} \mathrm{SO}_{4}$, the solid was filtered off, and the filtrate was evaporated under reduced pressure. The crude product was purified by column chromatography on silica gel using hexane/i-PrOH (10:1 $\mathrm{v} / \mathrm{v}$ ) as an eluent.

Benzophospholan-3-one Sulfide (4a). Compound 3a (0.48 g, 2.0 $\mathrm{mmol}$ ) was reacted according to general procedure $\mathrm{C}$ to afford 4 as a brownish oil $(67 \%, 0.346 \mathrm{~g}, 1.34 \mathrm{mmol}) . R_{f}=0.44(10: 1$ hexane $/ i-$ $\mathrm{PrOH}) .{ }^{1} \mathrm{H}$ NMR $\left(500 \mathrm{MHz}, \mathrm{CDCl}_{3}\right): \delta 8.03-8.07$ (m, $\left.1 \mathrm{H}\right), 7.83-$ $7.91(\mathrm{~m}, 2 \mathrm{H}), 7.74-7.78(\mathrm{~m}, 1 \mathrm{H}), 7.65-7.70(\mathrm{~m}, 2 \mathrm{H}), 7.52-7.53$ $(\mathrm{m}, 1 \mathrm{H}), 7.44-7.48(\mathrm{~m}, 2 \mathrm{H}), 3.35-3.47(\mathrm{~m}, 2 \mathrm{H}) .{ }^{13} \mathrm{C}\left\{{ }^{1} \mathrm{H}\right\}$ NMR $\left(125 \mathrm{MHz}, \mathrm{CDCl}_{3}\right): \delta 195.3\left(\mathrm{~d}, J_{\mathrm{C}-\mathrm{P}}=9.1 \mathrm{~Hz}, \mathrm{C}\right), 143.4\left(\mathrm{~d}, J_{\mathrm{C}-\mathrm{P}}=\right.$ $76.3 \mathrm{~Hz}, \mathrm{C}), 139.6\left(\mathrm{~d}, J_{\mathrm{C}-\mathrm{P}}=10.9 \mathrm{~Hz}, \mathrm{C}\right), 136.4\left(\mathrm{~d}, J_{\mathrm{C}-\mathrm{P}}=11.8 \mathrm{~Hz}\right.$, $\mathrm{CH}), 132.9\left(\mathrm{~d}, J_{\mathrm{C}-\mathrm{P}}=2.7 \mathrm{~Hz}, \mathrm{CH}\right), 132.1\left(\mathrm{~d}, J_{\mathrm{C}-\mathrm{P}}=3.6 \mathrm{~Hz}, \mathrm{CH}\right)$, $131.2\left(\mathrm{~d}, J_{\mathrm{C}-\mathrm{P}}=81.8 \mathrm{~Hz}, \mathrm{C}\right), 130.8\left(\mathrm{~d}, J_{\mathrm{C}-\mathrm{P}}=11.8 \mathrm{~Hz}, 2 \mathrm{CH}\right), 129.4$ $\left(\mathrm{d}, J_{\mathrm{C}-\mathrm{P}}=7.3 \mathrm{~Hz}, \mathrm{CH}\right), 128.9\left(\mathrm{~d}, J_{\mathrm{C}-\mathrm{P}}=12.7 \mathrm{~Hz}, 2 \mathrm{CH}\right), 128.3(\mathrm{~d}$, $\left.J_{\mathrm{C}-\mathrm{P}}=19.9 \mathrm{~Hz}, \mathrm{C}\right), 124.8\left(\mathrm{~d}, J_{\mathrm{C}-\mathrm{P}}=10.9 \mathrm{~Hz}, \mathrm{CH}\right), 45.8\left(\mathrm{~d}, J_{\mathrm{C}-\mathrm{P}}=\right.$ $\left.57.2 \mathrm{~Hz}, \mathrm{CH}_{2}\right) \cdot{ }^{31} \mathrm{P}\left\{{ }^{1} \mathrm{H}\right\} \mathrm{NMR}\left(202 \mathrm{MHz}, \mathrm{CDCl}_{3}\right): \delta 32.02(\mathrm{~s}) . \mathrm{GC}-$ MS (EI) $m / z: 260(7), 259(58)(\mathrm{M})^{+}, 258$ (100), 226 (15), 184 (10), 185 (33), 165 (24), 153 (11), 152 (31), 151 (7), 150 (12), 149 (98), 139 (21), 136 (10), 134 (12), 121 (36), 120 (12), 118 (11), 109 (44), 108 (24), 107 (67), 95 (13), 91 (29), 90 (15), 89 (20), 81 (19), 78 (20). Anal. Calcd for $\mathrm{C}_{14} \mathrm{H}_{11}$ OPS: C, 65.10; H, 4.29. Found: C, 64.90; H, 4.10.

Synthesis of Triflates 5 and $6 a$ (General Procedure D). To a Schlenk tube $(50 \mathrm{~mL})$ equipped with a magnetic stirrer and an argon inlet was added phosphine oxide $3(0.3 \mathrm{mmol})$ or sulfide 4 a $(0.3$ $\mathrm{mmol})$ in anhydrous DCM $(8 \mathrm{~mL})$, and the mixture was cooled to $-78{ }^{\circ} \mathrm{C}$ with a dry ice/acetone bath. DIPEA $(78.4 \mu \mathrm{L}, 0.45 \mathrm{mmol})$ was added dropwise. Then, $\mathrm{Tf}_{2} \mathrm{O}(61 \mu \mathrm{L}, 0.36 \mathrm{mmol})$ was added dropwise with vigorous stirring. The reaction mixture was stirred at
$-78{ }^{\circ} \mathrm{C}$ for $30 \mathrm{~min}$. The crude reaction mixture was checked using the ${ }^{31} \mathrm{P}\left\{{ }^{1} \mathrm{H}\right\}$ NMR technique. Then, the reaction mixture was quenched by the addition of $1 \mathrm{M} \mathrm{HCl}$ solution $(5 \mathrm{~mL})$ and extracted with $\mathrm{CHCl}_{3}(5 \times 10 \mathrm{~mL})$. The collected organic phases were dried over $\mathrm{Na}_{2} \mathrm{SO}_{4}$, the solid was filtered off, and the filtrate was evaporated under reduced pressure. The crude product was purified by column chromatography on silica gel using $\mathrm{CHCl}_{3} /$ acetone $(2: 1 \mathrm{v} / \mathrm{v})$ as an eluent.

1-Oxido-1-phenyl-1H-phosphindol-3-yl Trifluoromethanesulfonate $(5 \mathrm{a})$. Compound $3 \mathrm{a}(0.073 \mathrm{~g}, 0.3 \mathrm{mmol})$ was reacted according to general procedure $\mathrm{D}$ to afford $\mathbf{5 a}$ as an orange solid $(75 \%, 0.084 \mathrm{~g}$, $0.225 \mathrm{mmol}) . \mathrm{Mp}: 120.1-121.1{ }^{\circ} \mathrm{C} . R_{f}=0.76\left(2: 1 \mathrm{CHCl}_{3} /\right.$ acetone $)$. ${ }^{1} \mathrm{H}$ NMR $\left(500 \mathrm{MHz}, \mathrm{CDCl}_{3}\right): \delta 7.68-7.75(\mathrm{~m}, 3 \mathrm{H}), 7.62-7.66(\mathrm{~m}$, $1 \mathrm{H}), 7.56-7.61(\mathrm{~m}, 1 \mathrm{H}), 7.52-7.57(\mathrm{~m}, 2 \mathrm{H}), 7.46-7.50(\mathrm{~m}, 2 \mathrm{H})$, $6.31\left(\mathrm{~d}, J_{\mathrm{H}-\mathrm{P}}=14.82 \mathrm{~Hz}, 1 \mathrm{H}\right) .{ }^{13} \mathrm{C}\left\{{ }^{1} \mathrm{H}\right\} \operatorname{NMR}\left(125 \mathrm{MHz} \mathrm{CDCl}_{3}\right): \delta$ $156.7\left(\mathrm{~d}, J_{\mathrm{C}-\mathrm{P}}=34.5 \mathrm{~Hz}, \mathrm{C}\right), 135.8\left(\mathrm{~d}, J_{\mathrm{C}-\mathrm{P}}=19.1 \mathrm{~Hz}, \mathrm{C}\right), 133.4(\mathrm{~d}$, $\left.J_{\mathrm{C}-\mathrm{P}}=1.8 \mathrm{~Hz}, \mathrm{CH}\right), 133.1\left(\mathrm{~d}, J_{\mathrm{C}-\mathrm{P}}=2.7 \mathrm{~Hz}, \mathrm{CH}\right), 132.2\left(\mathrm{~d}, J_{\mathrm{C}-\mathrm{P}}=\right.$ $104.5 \mathrm{~Hz}, \mathrm{C}), 131.5\left(\mathrm{~d}, J_{\mathrm{C}-\mathrm{P}}=10.9 \mathrm{~Hz}, \mathrm{CH}\right), 130.8\left(\mathrm{~d}, J_{\mathrm{C}-\mathrm{P}}=10.9\right.$ $\mathrm{Hz}, 2 \mathrm{CH}), 129.5\left(\mathrm{~d}, J_{\mathrm{C}-\mathrm{P}}=8.2 \mathrm{~Hz}, \mathrm{CH}\right), 129.1\left(\mathrm{~d}, J_{\mathrm{C}-\mathrm{P}}=12.7 \mathrm{~Hz}\right.$, $2 \mathrm{CH}), 127.5\left(\mathrm{~d}, J_{\mathrm{C}-\mathrm{P}}=108.1 \mathrm{~Hz}, \mathrm{C}\right), 121.0\left(\mathrm{~d}, J_{\mathrm{C}-\mathrm{P}}=10.9 \mathrm{~Hz}, \mathrm{CH}\right)$, $118.5\left(\mathrm{q}, J_{\mathrm{C}-\mathrm{P}}=321.5 \mathrm{~Hz}, \mathrm{CF}_{3}\right), 108.9\left(\mathrm{~d}, J_{\mathrm{C}-\mathrm{P}}=95.4 \mathrm{~Hz}, \mathrm{CH}\right)$. ${ }^{31} \mathrm{P}\left\{{ }^{1} \mathrm{H}\right\}$ NMR $\left(202 \mathrm{MHz}, \mathrm{CDCl}_{3}\right): \delta 32.12(\mathrm{~s}) .{ }^{19} \mathrm{~F}$ NMR $(470 \mathrm{MHz}$, $\left.\mathrm{CDCl}_{3}\right): \delta-72.96$ (s). GC-MS (EI) $m / z: 374(5)(\mathrm{M})^{+}, 240(23)$, 194 (44), 183 (11), 179 (6), 178 (31), 177 (6), 166 (16), 165 (44), 152 (11), 151 (7), 107 (15), 89 (12), 77 (17), 69 (100). HRMS (ESI/Q-TOF) $m / z$ : calcd for $\mathrm{C}_{15} \mathrm{H}_{10} \mathrm{~F}_{3} \mathrm{O}_{4} \mathrm{PS}[\mathrm{M}+\mathrm{H}]^{+}, 375.0062$; found, 375.0053 .

1-Oxido-1-phenyl-1H-7-methylphosphindol-3-yl Trifluoromethanesulfonate $(\mathbf{5 b})$. Compound $3 \mathbf{b}(0.077 \mathrm{~g}, 0.3 \mathrm{mmol})$ was reacted according to general procedure $\mathrm{D}$ to afford $\mathbf{5 b}$ as a yellow solid (55\%, $0.064 \mathrm{~g}, 0.165 \mathrm{mmol})$. Mp: $125-126.5{ }^{\circ} \mathrm{C} . R_{f}=0.68(2: 1$ $\mathrm{CHCl}_{3}$ /acetone). ${ }^{1} \mathrm{H}$ NMR (500 $\left.\mathrm{MHz}, \mathrm{CDCl}_{3}\right): \delta 7.73-7.77(\mathrm{~m}$, $2 \mathrm{H}), 7.58-7.61(\mathrm{~m}, 1 \mathrm{H}), 7.47-7.53(\mathrm{~m}, 3 \mathrm{H}), 7.35-7.37(\mathrm{~m}, 1 \mathrm{H})$, 7.27-7.29 (m, 2H), $6.25\left(\mathrm{~d}, J_{\mathrm{H}-\mathrm{P}}=15.13 \mathrm{~Hz}, 1 \mathrm{H}\right), 2.37(\mathrm{~s}, 3 \mathrm{H})$. ${ }^{13} \mathrm{C}\left\{{ }^{1} \mathrm{H}\right\}$ NMR $\left(125 \mathrm{MHz}, \mathrm{CDCl}_{3}\right): \delta 156.8\left(\mathrm{~d}, J_{\mathrm{C}-\mathrm{P}}=34.5 \mathrm{~Hz}, \mathrm{C}\right)$, $142.2\left(\mathrm{~d}, J_{\mathrm{C}-\mathrm{P}}=8.2 \mathrm{~Hz}, \mathrm{C}\right), 135.8\left(\mathrm{~d}, J_{\mathrm{C}-\mathrm{P}}=19.1 \mathrm{~Hz}, \mathrm{C}\right), 133.5$ (d, $\left.J_{\mathrm{C}-\mathrm{P}}=2.7 \mathrm{~Hz}, \mathrm{CH}\right), 133.1\left(\mathrm{~d}, J_{\mathrm{C}-\mathrm{P}}=10.0 \mathrm{~Hz}, \mathrm{CH}\right), 132.9\left(\mathrm{~d}, J_{\mathrm{C}-\mathrm{P}}=\right.$ $2.7 \mathrm{~Hz}, \mathrm{CH}), 130.8\left(\mathrm{~d}, J_{\mathrm{C}-\mathrm{P}}=10.9 \mathrm{~Hz}, 2 \mathrm{CH}\right), 130.3\left(\mathrm{~d}, J_{\mathrm{C}-\mathrm{P}}=103.5\right.$ $\mathrm{Hz}, \mathrm{C}), 129.2\left(\mathrm{~d}, J_{\mathrm{C}-\mathrm{P}}=13.6 \mathrm{~Hz}, 2 \mathrm{CH}\right), 127.3\left(\mathrm{~d}, J_{\mathrm{C}-\mathrm{P}}=106.3 \mathrm{~Hz}\right.$ C), $118.5\left(\mathrm{q}, J_{\mathrm{C}-\mathrm{P}}=321.5 \mathrm{~Hz}, \mathrm{CF}_{3}\right), 118.48\left(\mathrm{~d}, J_{\mathrm{C}-\mathrm{P}}=10.9 \mathrm{~Hz}, \mathrm{CH}\right)$, $108.9\left(\mathrm{~d}, J_{\mathrm{C}-\mathrm{p}}=95.4 \mathrm{~Hz}, \mathrm{CH}\right), 19.4\left(\mathrm{~s}, \mathrm{CH}_{3}\right) \cdot{ }^{31} \mathrm{P}\left\{{ }^{1} \mathrm{H}\right\}$ NMR $(202$ $\left.\mathrm{MHz}, \mathrm{CDCl}_{3}\right): \delta 32.47(\mathrm{~s}) .{ }^{19} \mathrm{~F} \mathrm{NMR}\left(470 \mathrm{MHz}, \mathrm{CDCl}_{3}\right): \delta-72.98$ (s). GC-MS (EI) $m / z: 388$ (9) (M) ${ }^{+}, 255$ (9), 208 (33), 192 (18), 178 (16), 165 (26), 89 (13), 77 (19). HRMS (ESI/Q-TOF) $m / z$ : calcd for $\mathrm{C}_{16} \mathrm{H}_{12} \mathrm{O}_{4} \mathrm{~F}_{3} \mathrm{PS}[\mathrm{M}+\mathrm{H}]^{+}$, 389.0219; found, 389.0213.

1-Oxido-1-phenyl-1H-6-chlorophosphindol-3-yl Trifluoromethanesulfonate (5c). Compound $3 \mathrm{c}(0.083 \mathrm{~g}, 0.3 \mathrm{mmol})$ was reacted according to general procedure $\mathrm{D}$ to afford $\mathbf{5 c}$ as an orange oil $(60 \%$, $0.074 \mathrm{~g}, 0.18 \mathrm{mmol}) . R_{\mathrm{f}}=0.75\left(2: 1 \mathrm{CHCl}_{3}\right.$ /acetone) ${ }^{1} \mathrm{H}$ NMR (500 $\left.\mathrm{MHz}, \mathrm{CDCl}_{3}\right): \delta 7.71-7.75(\mathrm{~m}, 2 \mathrm{H}), 7.58-7.65(\mathrm{~m}, 3 \mathrm{H}), 7.45-7.52$ $(\mathrm{m}, 3 \mathrm{H}), 6.31\left(\mathrm{~d}, J_{\mathrm{H}-\mathrm{P}}=15.29 \mathrm{~Hz}, 1 \mathrm{H}\right) .{ }^{13} \mathrm{C}\left\{{ }^{1} \mathrm{H}\right\}$ NMR $(125 \mathrm{MHz}$, $\left.\mathrm{CDCl}_{3}\right): \delta 156.2\left(\mathrm{~d}, J_{\mathrm{C}-\mathrm{P}}=33.6 \mathrm{~Hz}, \mathrm{C}\right), 138.4\left(\mathrm{~d}, J_{\mathrm{C}-\mathrm{P}}=14.3 \mathrm{~Hz}, \mathrm{C}\right)$, $134.6\left(\mathrm{~d}, J_{\mathrm{C}-\mathrm{P}}=100.8 \mathrm{~Hz}, \mathrm{C}\right), 133.9\left(\mathrm{~d}, J_{\mathrm{C}-\mathrm{P}}=19.1 \mathrm{~Hz}, \mathrm{C}\right), 133.4(\mathrm{~d}$, $\left.J_{\mathrm{C}-\mathrm{P}}=2.7 \mathrm{~Hz}, \mathrm{CH}\right), 133.3\left(\mathrm{~d}, J_{\mathrm{C}-\mathrm{P}}=1.8 \mathrm{~Hz}, \mathrm{CH}\right), 130.8\left(\mathrm{~d}, J_{\mathrm{C}-\mathrm{P}}=\right.$ $10.9 \mathrm{~Hz}, 2 \mathrm{CH}), 128.9\left(\mathrm{~d}, J_{\mathrm{C}-\mathrm{P}}=10.0 \mathrm{~Hz}, \mathrm{CH}\right), 128.4\left(\mathrm{~d}, J_{\mathrm{C}-\mathrm{P}}=13.6\right.$ $\mathrm{Hz}, 2 \mathrm{CH}), 126.7\left(\mathrm{~d}, J_{\mathrm{C}-\mathrm{P}}=108.9 \mathrm{~Hz}, \mathrm{C}\right), 122.2\left(\mathrm{~d}, J_{\mathrm{C}-\mathrm{P}}=11.8 \mathrm{~Hz}\right.$, $\mathrm{CH}), 118.5\left(\mathrm{q}, J_{\mathrm{C}-\mathrm{P}}=320.6 \mathrm{~Hz}, \mathrm{CF}_{3}\right), 109.9\left(\mathrm{~d}, J_{\mathrm{C}-\mathrm{P}}=98.6 \mathrm{~Hz}, \mathrm{CH}\right)$. ${ }^{31} \mathrm{P}\left\{{ }^{1} \mathrm{H}\right\}$ NMR $\left(202 \mathrm{MHz}, \mathrm{CDCl}_{3}\right): \delta 31.06(\mathrm{~s}) .{ }^{19} \mathrm{~F}$ NMR $(470 \mathrm{MHz}$, $\mathrm{CDCl}_{3}$ ): $\delta-72.83$ (s). GC-MS (EI) $m / z: 411$ (3) 410 (10), 409 (5), $408(28)(\mathrm{M})^{+}, 277$ (16), 276 (16), 275 (51), 259 (22), 232 (11), 229 (30), 229 (20), 228 (100), 214 (30), 213 (14), 212 (92), 211 (11), 199 (22), 196 (22), 193 (36), 183 (13), 176 (16), 166 (16), 165 (75), 164 (14), 151 (24), 140 (11), 107 (21), 105 (28), 89 (12). HRMS (ESI/Q-TOF) $m / z$ : calcd for $\mathrm{C}_{15} \mathrm{H}_{9} \mathrm{ClF}_{3} \mathrm{O}_{4} \mathrm{PS}[\mathrm{M}+\mathrm{H}]^{+}$, 408.9673; found, 408.9681 .

1-Oxido-1-phenyl-1H-5-methoxyphosphindol-3-yl Trifluoromethanesulfonate $(5 d)$. Compound $3 \mathrm{c}(0.082 \mathrm{~g}, 0.3 \mathrm{mmol})$ was reacted according to general procedure $\mathrm{D}$ to afford $\mathbf{5 d}$ as an orange oil $(58 \%, 0.07 \mathrm{~g}, 0.174 \mathrm{mmol}) . R_{\mathrm{f}}=0.65\left(2: 1 \mathrm{CHCl}_{3} /\right.$ acetone $) .{ }^{1} \mathrm{H} \mathrm{NMR}$ $\left(500 \mathrm{MHz}, \mathrm{CDCl}_{3}\right): \delta 7.68-7.72(\mathrm{~m}, 2 \mathrm{H}), 7.55-7.62(\mathrm{~m}, 2 \mathrm{H})$, 
7.44-7.48 (m, 2H), 7.03-7.04 (m, 1H), 6.97-7.00 (m, 1H), $6.30(\mathrm{~d}$, $\left.J_{\mathrm{H}-\mathrm{P}}=14.66 \mathrm{~Hz}, 1 \mathrm{H}\right), 3.90(\mathrm{~s}, 3 \mathrm{H}) .{ }^{13} \mathrm{C}\left\{{ }^{1} \mathrm{H}\right\}$ NMR $(125 \mathrm{MHz}$, $\left.\mathrm{CDCl}_{3}\right): \delta 164.0\left(J_{\mathrm{P}-\mathrm{C}}=1.8 \mathrm{~Hz}, \mathrm{C}\right), 155.9\left(\mathrm{~d}, J_{\mathrm{P}-\mathrm{C}}=33.6 \mathrm{~Hz}, \mathrm{C}\right)$, $138.2\left(\mathrm{~d}, J_{\mathrm{P}-\mathrm{C}}=20.9 \mathrm{~Hz}, \mathrm{C}\right), 132.9\left(\mathrm{~d}, J_{\mathrm{P}-\mathrm{C}}=2.7 \mathrm{~Hz}, \mathrm{CH}\right), 131.1(\mathrm{~d}$, $\left.J_{\mathrm{P}-\mathrm{C}}=10.0 \mathrm{~Hz}, \mathrm{CH}\right), 130.8\left(\mathrm{~d}, J_{\mathrm{P}-\mathrm{C}}=11.8 \mathrm{~Hz}, 2 \mathrm{CH}\right), 129.1\left(\mathrm{~d}, J_{\mathrm{P}-\mathrm{C}}\right.$ $=13.6 \mathrm{~Hz}, 2 \mathrm{CH}), 129.7\left(\mathrm{~d}, J_{\mathrm{P}-\mathrm{C}}=108.9 \mathrm{~Hz}, \mathrm{C}\right), 122.7\left(\mathrm{~d}, J_{\mathrm{P}-\mathrm{C}}=\right.$ $110.8 \mathrm{~Hz}, \mathrm{C}), 118.6\left(\mathrm{q}, J_{\mathrm{P}-\mathrm{C}}=320.6 \mathrm{~Hz}, \mathrm{CF}_{3}\right), 115.5\left(\mathrm{~d}, J_{\mathrm{P}-\mathrm{C}}=12.7\right.$ $\mathrm{Hz}, \mathrm{CH}), 110.5\left(\mathrm{~d}, J_{\mathrm{P}-\mathrm{C}}=94.5 \mathrm{~Hz}, \mathrm{CH}\right), 108.0\left(\mathrm{~d}, J_{\mathrm{P}-\mathrm{C}}=11.8 \mathrm{~Hz}\right.$, $\mathrm{CH}), 55.8\left(\mathrm{~s}, \mathrm{CH}_{3}\right) .{ }^{31} \mathrm{P}\left\{{ }^{1} \mathrm{H}\right\} \mathrm{NMR}\left(202 \mathrm{MHz}, \mathrm{CDCl}_{3}\right): \delta 30.95$ (s). ${ }^{19} \mathrm{~F}$ NMR (470 MHz, $\mathrm{CDCl}_{3}$ ): $\delta-72.89$ (s). GC-MS (EI) $\mathrm{m} / z: 405$ (2), $404(11)(\mathrm{M})^{+}, 282(13), 281(33), 273(11), 272$ (62) (M$\left.\mathrm{SO}_{2} \mathrm{CF}_{3}\right), 271$ (57), 256 (72), 240 (20), 229 (24), 224 (71) 223 (18), 209 (38), 208 (94), 207 (100), 196 (15), 195 (25), 179 (49), 165 (29), 152 (34), 151 (18), 148 (18), 139 (24), 137 (18), 120 (36), 106 (24), 91 (47), 89 (19). HRMS (ESI/Q-TOF) $\mathrm{m} / z$ : calcd for $\mathrm{C}_{16} \mathrm{H}_{12} \mathrm{O}_{5} \mathrm{~F}_{3} \mathrm{PS}[\mathrm{M}+\mathrm{H}]^{+}$, 405.0168; found, 405.0165 .

1-Sulfido-1-phenyl-1H-phosphindol-3-yl Trifluoromethanesulfonate $(6 \mathrm{a})$. Compound $4 \mathrm{a}(0.077 \mathrm{~g}, 0.3 \mathrm{mmol})$ was reacted according to general procedure D to afford $\mathbf{6}$ as a yellowish solid $(50 \%, 0.058 \mathrm{~g}$, $0.15 \mathrm{mmol}) . \mathrm{Mp}: 109-110^{\circ} \mathrm{C} . R_{f}=0.59(10: 1$ hexane $/ i-\mathrm{PrOH}) .{ }^{1} \mathrm{H}$ NMR (500 MHz, $\left.\mathrm{CDCl}_{3}\right): \delta 7.78-7.82(\mathrm{~m}, 2 \mathrm{H}), 7.68-7.72(\mathrm{~m}, 2 \mathrm{H})$, $7.54-7.59(\mathrm{~m}, 3 \mathrm{H}), 7.44-7.48(\mathrm{~m}, 2 \mathrm{H}), 6.40\left(\mathrm{~d}, J_{\mathrm{H}-\mathrm{P}}=18.60 \mathrm{~Hz}\right.$, 1H). ${ }^{13} \mathrm{C}\left\{{ }^{1} \mathrm{H}\right\} \mathrm{NMR}\left(125 \mathrm{MHz}, \mathrm{CDCl}_{3}\right): \delta 154.7\left(\mathrm{~d}, J_{\mathrm{P}-\mathrm{C}}=28.9 \mathrm{~Hz}\right.$, C), $135.59\left(\mathrm{~d}, J_{\mathrm{P}-\mathrm{C}}=87.9 \mathrm{~Hz}, \mathrm{C}\right), 135.58\left(\mathrm{~d}, J_{\mathrm{P}-\mathrm{C}}=16.2 \mathrm{~Hz}, \mathrm{C}\right)$, $132.8\left(\mathrm{~d}, J_{\mathrm{P}-\mathrm{C}}=3.1 \mathrm{~Hz}, \mathrm{CH}\right), 132.7\left(\mathrm{~d}, J_{\mathrm{P}-\mathrm{C}}=1.8 \mathrm{~Hz}, \mathrm{CH}\right), 133.2(\mathrm{~d}$, $\left.J_{\mathrm{P}-\mathrm{C}}=11.7 \mathrm{~Hz}, \mathrm{CH}\right), 130.8\left(\mathrm{~d}, J_{\mathrm{P}-\mathrm{C}}=12.7 \mathrm{~Hz}, \mathrm{CH}\right), 129.1\left(\mathrm{~d}, J_{\mathrm{P}-\mathrm{C}}=\right.$ $12.9 \mathrm{~Hz}, \mathrm{CH}), 128.9\left(\mathrm{~d}, J_{\mathrm{P}-\mathrm{C}}=9.2 \mathrm{~Hz}, \mathrm{CH}\right), 127.4\left(\mathrm{~d}, J_{\mathrm{P}-\mathrm{C}}=84.4\right.$ $\mathrm{Hz}, \mathrm{C}), 121.3\left(\mathrm{~d}, J_{\mathrm{P}-\mathrm{C}}=10.4 \mathrm{~Hz}, \mathrm{CH}\right), 118.5\left(\mathrm{q}, J_{\mathrm{P}-\mathrm{C}}=321.4 \mathrm{~Hz}\right.$, $\left.\mathrm{CF}_{3}\right), 112.4\left(\mathrm{~d}, J_{\mathrm{P}-\mathrm{C}}=78.8 \mathrm{~Hz}, \mathrm{CH}\right) .{ }^{31} \mathrm{P}\left\{{ }^{1} \mathrm{H}\right\}$ NMR $(202 \mathrm{MHz}$, $\left.\mathrm{CDCl}_{3}\right): \delta 41.0(\mathrm{~s}) .{ }^{19} \mathrm{~F}$ NMR $\left(470 \mathrm{MHz}, \mathrm{CDCl}_{3}\right): \delta-72.92(\mathrm{~s})$. GC-MS (EI) $m / z: 391$ (7), 390 (34), 258 (22), 257 (100), 239 (14), 229 (31), 225 (21), 210 (17), 197 (27), 196 (47), 195 (15), 194 (37), 193 (17), 182 (26), 178 (49), 166 (25), 165 (82), 152 (39). HRMS (ESI/Q-TOF) $m / z$ : calcd for $\mathrm{C}_{15} \mathrm{H}_{10} \mathrm{O}_{3} \mathrm{~F}_{3} \mathrm{PS}_{2}[\mathrm{M}+\mathrm{H}]^{+}$, 390.9834; found, 390.9834.

Reaction of $\mathbf{5}$ or $\mathbf{6}$ with Aryl Grignard Reagents (General Procedure E). To a Schlenk tube $(25 \mathrm{~mL})$ equipped with a magnetic stirrer and an argon inlet was added phosphine oxide 5 or $\mathbf{6}(0.13$ $\mathrm{mmol})$ in anhydrous THF $(2 \mathrm{~mL})$, and the mixture was cooled to 0 ${ }^{\circ} \mathrm{C}$. Then, aryl Grignard reagent $(0.195 \mathrm{mmol})$ was added. Then, the reaction mixture was stirred at $0{ }^{\circ} \mathrm{C}$ for $30-60 \mathrm{~min}$. Then, the reaction mixture was quenched by the addition of $\mathrm{NH}_{4} \mathrm{Cl}$ solution (5 $\mathrm{mL})$ and extracted with $\mathrm{CHCl}_{3}(5 \times 5 \mathrm{~mL})$. The collected organic phases were dried over $\mathrm{Na}_{2} \mathrm{SO}_{4}$, the solid was filtered off, and the was filtrate evaporated under reduced pressure. The crude reaction mixture was checked using the NMR technique. The residue was purified by column chromatography on silica gel using $\mathrm{CHCl}_{3}$ / EtOAc/MeOH (30:5:1 v/v) or $\mathrm{CHCl}_{3} / \mathrm{MTBE}(30: 1 \mathrm{v} / \mathrm{v})$ as an eluent.

(2-Ethynylphenyl)phenyl(p-tolyl)phosphine Oxide (8a). Compound 5a $(0.0486 \mathrm{~g}, 0.13 \mathrm{mmol})$ was reacted with $p$-TolMgBr ( $0.195 \mathrm{~mL}, 0.195 \mathrm{mmol}, 1 \mathrm{M}$ solution in THF) according to general procedure $\mathrm{E}$ for $30 \mathrm{~min}$ to afford $8 \mathrm{a}(0.0403 \mathrm{~g}, 0.127 \mathrm{mmol}, 98 \%$ yield) as a yellowish solid. Mp: $173-174{ }^{\circ} \mathrm{C} . R_{f}=0.45$ (30:5:1 $\left.\mathrm{CHCl}_{3} / \mathrm{AcOEt} / \mathrm{MeOH}\right) .{ }^{1} \mathrm{H}$ NMR $\left(500 \mathrm{MHz}, \mathrm{CDCl}_{3}\right): \delta 7.71-7.75$ (m, 2H), 7.59-7.68 (m, 4H), 7.40-7.56 (m, 5H), 7.26-7.28 (m, $2 \mathrm{H}), 3.00(\mathrm{~s}, 1 \mathrm{H}), 2.41(\mathrm{~s}, 3 \mathrm{H}) .{ }^{13} \mathrm{C}\left\{{ }^{1} \mathrm{H}\right\} \operatorname{NMR}\left(125 \mathrm{MHz}, \mathrm{CDCl}_{3}\right)$ : $\delta 142.3\left(\mathrm{~d}, J_{\mathrm{P}-\mathrm{C}}=2.7 \mathrm{~Hz}, \mathrm{C}\right), 134.9\left(\mathrm{~d}, J_{\mathrm{P}-\mathrm{C}}=100.8 \mathrm{~Hz}, \mathrm{C}\right), 134.9(\mathrm{~d}$, $\left.J_{\mathrm{P}-\mathrm{C}}=9.1 \mathrm{~Hz}, \mathrm{CH}\right), 133.8\left(\mathrm{~d}, J_{\mathrm{P}-\mathrm{C}}=9.1 \mathrm{~Hz}, \mathrm{CH}\right), 132.5\left(\mathrm{~d}, J_{\mathrm{P}-\mathrm{C}}=\right.$ $106.3 \mathrm{~Hz}, \mathrm{C}), 132.3\left(\mathrm{~d}, J_{\mathrm{P}-\mathrm{C}}=10.9 \mathrm{~Hz}, 2 \mathrm{CH}\right), 132.2\left(\mathrm{~d}, J_{\mathrm{P}-\mathrm{C}}=10.0\right.$ $\mathrm{Hz}, 2 \mathrm{CH}), 131.7\left(\mathrm{~d}, J_{\mathrm{P}-\mathrm{C}}=2.7 \mathrm{~Hz}, \mathrm{CH}\right), 131.6\left(\mathrm{~d}, J_{\mathrm{P}-\mathrm{C}}=1.8 \mathrm{~Hz}\right.$, $\mathrm{CH}), 129.1\left(\mathrm{~d}, J_{\mathrm{P}-\mathrm{C}}=12.7 \mathrm{~Hz}, 2 \mathrm{CH}\right), 128.7\left(\mathrm{~d}, J_{\mathrm{P}-\mathrm{C}}=108.9 \mathrm{~Hz}, \mathrm{C}\right)$, $128.4\left(\mathrm{~d}, J_{\mathrm{P}-\mathrm{C}}=11.8 \mathrm{~Hz}, \mathrm{CH}\right), 128.3\left(\mathrm{~d}, J_{\mathrm{P}-\mathrm{C}}=11.8 \mathrm{~Hz}, \mathrm{CH}\right), 125.3$ $\left(\mathrm{d}, J_{\mathrm{P}-\mathrm{C}}=7.3 \mathrm{~Hz}, \mathrm{C}\right), 85.0(\mathrm{~s}, \mathrm{C}), 81.5\left(\mathrm{~d}, J_{\mathrm{P}-\mathrm{C}}=5.5 \mathrm{~Hz}, \mathrm{CH}\right), 21.6$ $\left(\mathrm{s}, \mathrm{CH}_{3}\right) \cdot{ }^{31} \mathrm{P}\left\{{ }^{1} \mathrm{H}\right\}$ NMR $\left(202 \mathrm{MHz}, \mathrm{CDCl}_{3}\right): \delta 28.70(\mathrm{~s})$. GC-MS (EI) $m / z: 317$ (21), $316(93)(\mathrm{M})^{+}, 315$ (100), 297 (15), 269 (13), 268 (15), 254 (18), 253 (18), 252 (15), 239 (34), 223 (17), 215 (11), 210 (12), 197 (11), 196 (19), 191 (11), 189 (11), 182 (15), 174 (17), 165 (32), 152 (21), 91 (24), 89 (12), 78 (15). HRMS
(ESI/Q-TOF) $m / z$ : calcd for $\mathrm{C}_{21} \mathrm{H}_{17} \mathrm{OP}[\mathrm{M}+\mathrm{H}]^{+}, 317.1090$; found, 317.1088 .

(2-Ethynylphenyl)phenyl(m-tolyl)phosphine Oxide (8b). Compound $5 \mathrm{a}(0.0486 \mathrm{~g}, 0.13 \mathrm{mmol})$ was reacted with $m$-TolMgBr $(0.195$ $\mathrm{mL}, 0.195 \mathrm{mmol}, 1 \mathrm{M}$ solution in THF) according to general procedure E for $30 \mathrm{~min}$ to afford $\mathbf{8 b}(0.0345 \mathrm{~g}, 0.109 \mathrm{mmol}$, $84 \%$ yield) as a yellowish oil. $R_{f}=0.65\left(2: 1 \mathrm{CHCl}_{3}\right.$ /acetone $) .{ }^{1} \mathrm{H}$ NMR $\left(500 \mathrm{MHz}, \mathrm{CDCl}_{3}\right): \delta 7.71-7.75(\mathrm{~m}, 2 \mathrm{H}), 7.60-7.68(\mathrm{~m}, 3 \mathrm{H})$, 7.39-7.58 (m, 6H), 7.33-7.36 (m, 2H), $3.00(\mathrm{~s}, 1 \mathrm{H}), 2.37(\mathrm{~s}, 3 \mathrm{H})$. ${ }^{13} \mathrm{C}\left\{{ }^{1} \mathrm{H}\right\} \operatorname{NMR}\left(125 \mathrm{MHz}, \mathrm{CDCl}_{3}\right): \delta 138.2\left(\mathrm{~d}, J_{\mathrm{P}-\mathrm{C}}=11.8 \mathrm{~Hz}, \mathrm{C}\right)$, $134.9\left(\mathrm{~d}, J_{\mathrm{P}-\mathrm{C}}=8.2 \mathrm{~Hz}, \mathrm{CH}\right), 134.7\left(\mathrm{~d}, J_{\mathrm{P}-\mathrm{C}}=100.8 \mathrm{~Hz}, \mathrm{C}\right), 133.8(\mathrm{~d}$, $\left.J_{\mathrm{P}-\mathrm{C}}=9.1 \mathrm{~Hz}, \mathrm{CH}\right), 132.6\left(\mathrm{~d}, J_{\mathrm{P}-\mathrm{C}}=1.8 \mathrm{~Hz}, \mathrm{CH}\right), 132.59\left(\mathrm{~d}, J_{\mathrm{P}-\mathrm{C}}=\right.$ $10.0 \mathrm{~Hz}, \mathrm{CH}), 132.4\left(\mathrm{~d}, J_{\mathrm{P}-\mathrm{C}}=105.4 \mathrm{~Hz}, \mathrm{C}\right), 132.15\left(\mathrm{~d}, J_{\mathrm{P}-\mathrm{C}}=10.0\right.$ $\mathrm{Hz}, 2 \mathrm{CH}), 134.7\left(\mathrm{~d}, J_{\mathrm{P}-\mathrm{C}}=100.8 \mathrm{~Hz}, \mathrm{C}\right), 131.8\left(\mathrm{~d}, J_{\mathrm{P}-\mathrm{C}}=105.4 \mathrm{~Hz}\right.$, C), $131.7\left(\mathrm{~d}, J_{\mathrm{P}-\mathrm{C}}=2.7 \mathrm{~Hz}, \mathrm{CH}\right), 131.6\left(\mathrm{~d}, J_{\mathrm{P}-\mathrm{C}}=1.8 \mathrm{~Hz}, \mathrm{CH}\right), 129.3$ $\left(\mathrm{d}, J_{\mathrm{P}-\mathrm{C}}=10.9 \mathrm{~Hz}, \mathrm{CH}\right), 128.4\left(\mathrm{~d}, J_{\mathrm{P}-\mathrm{C}}=11.8 \mathrm{~Hz}, \mathrm{CH}\right), 128.3(\mathrm{~d}$, $\left.J_{\mathrm{P}-\mathrm{C}}=12.7 \mathrm{~Hz}, 2 \mathrm{CH}\right), 128.1\left(\mathrm{~d}, J_{\mathrm{P}-\mathrm{C}}=13.6 \mathrm{~Hz}, \mathrm{CH}\right), 125.3\left(\mathrm{~d}, J_{\mathrm{P}-\mathrm{C}}\right.$ $=6.4 \mathrm{~Hz}, \mathrm{C}), 84.9(\mathrm{~s}, \mathrm{C}), 81.6\left(\mathrm{~d}, J_{\mathrm{P}-\mathrm{C}}=6.4 \mathrm{~Hz}, \mathrm{CH}\right), 21.5\left(\mathrm{~s}, \mathrm{CH}_{3}\right)$. ${ }^{31} \mathrm{P}\left\{{ }^{1} \mathrm{H}\right\}$ NMR $\left(202 \mathrm{MHz}, \mathrm{CDCl}_{3}\right): \delta 28.56(\mathrm{~s})$. GC-MS (EI) $\mathrm{m} / z$ : 317 (22), $316(100)(\mathrm{M})^{+}, 315$ (97), 268 (20), 267 (13), 254 (11), 253 (24), 252 (17), 239 (28), 210 (15), 207 (11), 197 (14), 196 (35), 195 (11), 194 (10), 189 (12), 183 (14), 178 (26), 176 (19), 166 (11), 165 (48), 153 (12), 152 (42), 151 (16), 150 (12), 107 (16), 102 (12), 101 (12), 92 (35), 91 (67), 89 (40). HRMS (ESI/QTOF) $m / z$ : calcd for $\mathrm{C}_{21} \mathrm{H}_{17} \mathrm{OP}[\mathrm{M}+\mathrm{Na}]^{+}, 339.0909$; found, 339.0912 .

(2-Ethynylphenyl)phenyl(o-tolyl)phosphine Oxide (8c). Compound $5 \mathrm{a}(0.0486 \mathrm{~g}, 0.13 \mathrm{mmol})$ was reacted with $o$-TolMgCl (0.195 mL, $0.195 \mathrm{mmol}, 1 \mathrm{M}$ solution in THF) according to general procedure $\mathrm{E}$ for $30 \mathrm{~min}$ to afford $8 \mathrm{c}(0.0156 \mathrm{~g}, 0.0494 \mathrm{mmol}, 38 \%$ yield) as a yellowish oil. $R_{f}=0.15\left(30: 1 \mathrm{CHCl}_{3} / \mathrm{MTBE}\right) .{ }^{1} \mathrm{H}$ NMR $\left(500 \mathrm{MHz}, \mathrm{CDCl}_{3}\right): \delta$ 7.75-7.82 (m, 3H), 7.61-7.63 (m, $\left.1 \mathrm{H}\right)$, $7.52-7.57(\mathrm{~m}, 2 \mathrm{H}), 7.41-7.49(\mathrm{~m}, 5 \mathrm{H}), 7.18-7.20(\mathrm{~m}, 1 \mathrm{H}), 7.13-$ $7.16(\mathrm{~m}, 1 \mathrm{H}), 2.94(\mathrm{~s}, 1 \mathrm{H}), 2.47(\mathrm{~s}, 3 \mathrm{H}) .{ }^{13} \mathrm{C}\left\{{ }^{1} \mathrm{H}\right\}$ NMR $(125 \mathrm{MHz}$, $\left.\mathrm{CDCl}_{3}\right): \delta 143.2\left(\mathrm{~d}, J_{\mathrm{P}-\mathrm{C}}=8.2 \mathrm{~Hz}, \mathrm{C}\right), 135.0\left(\mathrm{~d}, J_{\mathrm{P}-\mathrm{C}}=99.9 \mathrm{~Hz}, \mathrm{C}\right)$, $134.9\left(\mathrm{~d}, J_{\mathrm{P}-\mathrm{C}}=9.1 \mathrm{~Hz}, \mathrm{CH}\right), 133.7\left(\mathrm{~d}, J_{\mathrm{P}-\mathrm{C}}=12.7 \mathrm{~Hz}, \mathrm{CH}\right), 133.4$ $\left(\mathrm{d}, J_{\mathrm{P}-\mathrm{C}}=9.1 \mathrm{~Hz}, \mathrm{CH}\right), 132.4\left(\mathrm{~d}, J_{\mathrm{P}-\mathrm{C}}=10.0 \mathrm{~Hz}, \mathrm{CH}\right), 131.9\left(\mathrm{~d}, J_{\mathrm{P}-\mathrm{C}}\right.$ $=2.7 \mathrm{~Hz}, \mathrm{CH}), 131.8\left(\mathrm{~d}, J_{\mathrm{P}-\mathrm{C}}=2.7 \mathrm{~Hz}, \mathrm{CH}\right), 131.55\left(\mathrm{~d}, J_{\mathrm{P}-\mathrm{C}}=10.9\right.$ $\mathrm{Hz}, \mathrm{CH}), 131.51\left(\mathrm{~d}, J_{\mathrm{P}-\mathrm{C}}=7.3 \mathrm{~Hz}, \mathrm{CH}\right), 130.4\left(\mathrm{~d}, J_{\mathrm{P}-\mathrm{C}}=105.4 \mathrm{~Hz}\right.$, C), $128.7\left(\mathrm{~d}, J_{\mathrm{P}-\mathrm{C}}=11.8 \mathrm{~Hz}, \mathrm{CH}\right), 128.3\left(\mathrm{~d}, J_{\mathrm{P}-\mathrm{C}}=12.7 \mathrm{~Hz}, \mathrm{CH}\right)$, $124.9\left(\mathrm{~d}, J_{\mathrm{P}-\mathrm{C}}=12.7 \mathrm{~Hz}, \mathrm{CH}\right), 124.9\left(\mathrm{~d}, J_{\mathrm{P}-\mathrm{C}}=7.3 \mathrm{~Hz}, \mathrm{C}\right), 84.7(\mathrm{~s}$, C), $81.4\left(\mathrm{~d}, J_{\mathrm{P}-\mathrm{C}}=5.5 \mathrm{~Hz}, \mathrm{CH}\right), 21.7\left(\mathrm{~s}, \mathrm{CH}_{3}\right) .{ }^{31} \mathrm{P} \mathrm{NMR}(202 \mathrm{MHz}$, $\left.\mathrm{CDCl}_{3}\right): \delta 30.71$ (s). GC-MS (EI) $m / z: 317(8), 316(48)(\mathrm{M})^{+}$, 315 (100), 237 (17), 220 (11), 191 (16), 189 (14), 178 (11), 165 (21), 77 (11). HRMS (ESI/Q-TOF) $m / z$ : calcd for $\mathrm{C}_{21} \mathrm{H}_{17}$ OP $[2 \mathrm{M}+$ $\mathrm{Na}]^{+}, 655.1926$; found, 655.1929.

[2-(Ethynyl)phenyl]diphenylphosphine Oxide (8d). Compound 5a $(0.0486 \mathrm{~g}, 0.13 \mathrm{mmol})$ was reacted with $\mathrm{PhMgBr}(0.195 \mathrm{~mL}, 0.195$ $\mathrm{mmol}, 1 \mathrm{M}$ solution in THF) according to general procedure $\mathrm{E}$ for 30 min to afford $8 \mathbf{d}(0.0373 \mathrm{~g}, 0.124 \mathrm{mmol}, 95 \%$ yield $)$ as a yellowish solid. Mp: $181.9-182.9^{\circ} \mathrm{C} . R_{f}=0.59\left(2: 1 \mathrm{CHCl}_{3}\right.$ /acetone $) .{ }^{1} \mathrm{H}$ NMR $\left(500 \mathrm{MHz}, \mathrm{CDCl}_{3}\right): \delta 7.72-7.77(\mathrm{~m}, 4 \mathrm{H}), 7.65-7.69(\mathrm{~m}, 1 \mathrm{H})$, 7.61-7.62 (m, 1H), 7.49-7.59 (m, 3H), 7.41-7.48 (m, 5H), $2.99(\mathrm{~s}$, 1H). ${ }^{13} \mathrm{C}\left\{{ }^{1} \mathrm{H}\right\}$ NMR $\left(125 \mathrm{MHz}, \mathrm{CDCl}_{3}\right): \delta 134.9\left(\mathrm{~d}, J_{\mathrm{P}-\mathrm{C}}=9.1 \mathrm{~Hz}\right.$, $\mathrm{CH}), 134.7\left(\mathrm{~d}, J_{\mathrm{P}-\mathrm{C}}=100.8 \mathrm{~Hz}, \mathrm{C}\right), 133.8\left(\mathrm{~d}, J_{\mathrm{P}-\mathrm{C}}=10.0 \mathrm{~Hz}, \mathrm{CH}\right)$, $132.2\left(\mathrm{~d}, J_{\mathrm{P}-\mathrm{C}}=10.0 \mathrm{~Hz}, \mathrm{CH}\right), 132.1\left(\mathrm{~d}, J_{\mathrm{P}-\mathrm{C}}=106.2 \mathrm{~Hz}, \mathrm{C}\right), 131.8$ $\left(\mathrm{d}, J_{\mathrm{P}-\mathrm{C}}=2.7 \mathrm{~Hz}, \mathrm{CH}\right), 131.6\left(\mathrm{~d}, J_{\mathrm{P}-\mathrm{C}}=2.7 \mathrm{~Hz}, \mathrm{CH}\right), 128.5\left(\mathrm{~d}, J_{\mathrm{P}-\mathrm{C}}\right.$ $=11.8 \mathrm{~Hz}, \mathrm{CH}), 128.3\left(\mathrm{~d}, J_{\mathrm{P}-\mathrm{C}}=12.7 \mathrm{~Hz}, \mathrm{CH}\right), 125.3\left(\mathrm{~d}, J_{\mathrm{P}-\mathrm{C}}=6.4\right.$ $\mathrm{Hz}, \mathrm{C}), 85.0(\mathrm{~s}, \mathrm{C}), 81.5\left(\mathrm{~d}, \mathrm{~J}_{\mathrm{P}-\mathrm{C}}=5.5 \mathrm{~Hz}, \mathrm{CH}\right) .{ }^{31} \mathrm{P}$ NMR $(202$ $\mathrm{MHz}, \mathrm{CDCl}_{3}$ ): $\delta 28.49$ (s). GC-MS (EI) $\mathrm{m} / z: 303$ (12), 302 (50) $(\mathrm{M})^{+}, 301$ (46), 254 (12), 253 (12), 252 (12), 225 (17), 196 (24), 183 (16), 178 (17), 176 (14), 165 (31), 152 (27), 151 (15), 107 (13), 101 (11), 78 (44), 77 (93), 76 (15), 75 (33), 74 (17). HRMS (ESI/Q-TOF) $m / z$ : calcd for $\mathrm{C}_{20} \mathrm{H}_{15} \mathrm{OP}[\mathrm{M}+\mathrm{Na}]^{+}, 325.0753$; found, 325.0754

p-Anisyl(2-ethynylphenyl)phenylphosphine Oxide (8e). Compound 5a $(0.0486 \mathrm{~g}, 0.13 \mathrm{mmol})$ was reacted with $p-A n M g B r(0.39$ $\mathrm{mL}, 0.195 \mathrm{mmol}, 0.5 \mathrm{M}$ solution in THF) according to general procedure E for $30 \mathrm{~min}$ to afford $8 \mathrm{e}(0.0419 \mathrm{~g}, 0.127 \mathrm{mmol}, 98 \%$ yield $)$ as a yellowish solid. $\mathrm{Mp}: 149.5-150.5{ }^{\circ} \mathrm{C} . R_{f}=0.58(2: 1$ 
$\mathrm{CHCl}_{3}$ /acetone). ${ }^{1} \mathrm{H}$ NMR $\left(500 \mathrm{MHz}, \mathrm{CDCl}_{3}\right): \delta 7.65-7.76(\mathrm{~m}$, $5 \mathrm{H}), 7.61-7.62(\mathrm{~m}, 1 \mathrm{H}), 7.49-7.51(\mathrm{~m}, 2 \mathrm{H}), 7.41-7.48(\mathrm{~m}, 3 \mathrm{H})$, 6.97-6.99 (m, 2H), $3.89(\mathrm{~s}, 3 \mathrm{H}), 3.01(\mathrm{~s}, 1 \mathrm{H}) .{ }^{13} \mathrm{C}\left\{{ }^{1} \mathrm{H}\right\}$ NMR $(125$ $\left.\mathrm{MHz}, \mathrm{CDCl}_{3}\right): \delta 162.5\left(\mathrm{~d}, J_{\mathrm{P}-\mathrm{C}}=2.7 \mathrm{~Hz}, \mathrm{C}\right), 134.9\left(\mathrm{~d}, J_{\mathrm{P}-\mathrm{C}}=9.1 \mathrm{~Hz}\right.$, $\mathrm{CH}), 134.7\left(\mathrm{~d}, J_{\mathrm{P}-\mathrm{C}}=100.8 \mathrm{~Hz}, \mathrm{C}\right), 134.2\left(\mathrm{~d}, J_{\mathrm{P}-\mathrm{C}}=10.9 \mathrm{~Hz}, 2 \mathrm{CH}\right)$, $133.8\left(\mathrm{~d}, J_{\mathrm{P}-\mathrm{C}}=9.1 \mathrm{~Hz}, \mathrm{CH}\right), 132.3\left(\mathrm{~d}, J_{\mathrm{P}-\mathrm{C}}=107.2 \mathrm{~Hz}, \mathrm{C}\right), 132.2(\mathrm{~d}$, $\left.J_{\mathrm{P}-\mathrm{C}}=10.0 \mathrm{~Hz}, 2 \mathrm{CH}\right), 131.8\left(\mathrm{~d}, J_{\mathrm{P}-\mathrm{C}}=2.7 \mathrm{~Hz}, \mathrm{CH}\right), 131.7\left(\mathrm{~d}, J_{\mathrm{P}-\mathrm{C}}=\right.$ $2.7 \mathrm{~Hz}, \mathrm{CH}), 128.5\left(\mathrm{~d}, J_{\mathrm{P}-\mathrm{C}}=10.9 \mathrm{~Hz}, \mathrm{CH}\right), 128.3\left(\mathrm{~d}, J_{\mathrm{P}-\mathrm{C}}=11.8\right.$ $\mathrm{Hz}, 2 \mathrm{CH}), 125.2\left(\mathrm{~d}, J_{\mathrm{P}-\mathrm{C}}=7.3 \mathrm{~Hz}, \mathrm{C}\right), 122.8\left(\mathrm{~d}, J_{\mathrm{P}-\mathrm{C}}=112.6 \mathrm{~Hz}, \mathrm{C}\right)$, $113.9\left(\mathrm{~d}, J_{\mathrm{P}-\mathrm{C}}=13.6 \mathrm{~Hz}, 2 \mathrm{CH}\right), 85.0(\mathrm{~s}, \mathrm{C}), 81.6\left(\mathrm{~d}, J_{\mathrm{P}-\mathrm{C}}=5.5 \mathrm{~Hz}\right.$, $\mathrm{CH}), 55.3\left(\mathrm{~s}, \mathrm{CH}_{3}\right) \cdot{ }^{31} \mathrm{P}\left\{{ }^{1} \mathrm{H}\right\} \mathrm{NMR}\left(202 \mathrm{MHz}, \mathrm{CDCl}_{3}\right): \delta 28.15$ (s). GC-MS (EI) $m / z: 333$ (22), 332 (100) (M)+, 331 (72), 317 (19), 284 (14), 270 (15), 255 (24), 253 (14), 252 (12), 241 (11), 240 (10), 239 (20), 231 (10), 226 (15), 196 (15), 194 (15), 184 (11), 183 (36), 178 (13), 176 (17), 170 (16), 169 (10), 165 (52), 163 (12), 157 (10), 152 (30), 151 (12), 141 (12), 139 (18), 125 (25), 120 (15), 15 (15), 108 (11), 107 (19), 101 (10), 95 (17), 92 (41), 78 (34), 77 (34), 76 (18). HRMS (ESI/Q-TOF) $m / z$ : calcd for $\mathrm{C}_{21} \mathrm{H}_{17} \mathrm{O}_{2} \mathrm{P}[\mathrm{M}+\mathrm{Na}]^{+}$, 355.0856; found, 355.0856.

m-Anisyl(2-ethynylphenyl)phenylphosphine Oxide (8f). Compound 5a $(0.0486 \mathrm{~g}, 0.13 \mathrm{mmol})$ was reacted with $m-\mathrm{AnMgBr}$ ( $0.195 \mathrm{~mL}, 0.195 \mathrm{mmol}, 1 \mathrm{M}$ solution in THF) according to general procedure $\mathrm{E}$ for $30 \mathrm{~min}$ to afford $\mathbf{8 f}(0.0385 \mathrm{~g}, 0.116 \mathrm{mmol}, 89 \%$ yield $)$ as a yellow solid. $\mathrm{Mp}: 129-130{ }^{\circ} \mathrm{C} . R_{f}=0.69\left(2: 1 \mathrm{CHCl}_{3} /\right.$ acetone). ${ }^{1} \mathrm{H}$ NMR $\left(500 \mathrm{MHz}, \mathrm{CDCl}_{3}\right): \delta 7.71-7.77(\mathrm{~m}, 2 \mathrm{H}), 7.60-$ $7.64(\mathrm{~m}, 2 \mathrm{H}), 7.50-7.57(\mathrm{~m}, 2 \mathrm{H}), 7.44-7.49(\mathrm{~m}, 3 \mathrm{H}), 7.39-7.43$ (m, 2H), 7.23-7.27 (m, 1H), 7.07-7.09 (m, 1H), $3.80(\mathrm{~s}, 3 \mathrm{H}), 3.02$ $(\mathrm{s}, 1 \mathrm{H}) \cdot{ }^{13} \mathrm{C}\left\{{ }^{1} \mathrm{H}\right\} \operatorname{NMR}\left(125 \mathrm{MHz}, \mathrm{CDCl}_{3}\right): \delta 159.4\left(\mathrm{~d}, J_{\mathrm{P}-\mathrm{C}}=14.5\right.$ $\mathrm{Hz}, \mathrm{C}), 134.9\left(\mathrm{~d}, J_{\mathrm{P}-\mathrm{C}}=9.1 \mathrm{~Hz}, \mathrm{CH}\right), 133.8\left(\mathrm{~d}, J_{\mathrm{P}-\mathrm{C}}=9.9 \mathrm{~Hz}, \mathrm{CH}\right)$, $133.3\left(\mathrm{~d}, J_{\mathrm{P}-\mathrm{C}}=105.4 \mathrm{~Hz}, \mathrm{C}\right), 132.1\left(\mathrm{~d}, J_{\mathrm{P}-\mathrm{C}}=9.9 \mathrm{~Hz}, 2 \mathrm{CH}\right), 132.0$ $\left(\mathrm{d}, J_{\mathrm{P}-\mathrm{C}}=103.5 \mathrm{~Hz}, \mathrm{C}\right), 131.8\left(\mathrm{~d}, J_{\mathrm{P}-\mathrm{C}}=3.6 \mathrm{~Hz}, \mathrm{CH}\right), 131.7\left(\mathrm{~d}, J_{\mathrm{P}-\mathrm{C}}\right.$ $=1.8 \mathrm{~Hz}, \mathrm{CH}), 129.5\left(\mathrm{~d}, J_{\mathrm{P}-\mathrm{C}}=15.4 \mathrm{~Hz}, \mathrm{CH}\right), 128.4\left(\mathrm{~d}, J_{\mathrm{P}-\mathrm{C}}=9.9\right.$ $\mathrm{Hz}, \mathrm{CH}), 128.3\left(\mathrm{~d}, J_{\mathrm{P}-\mathrm{C}}=12.7 \mathrm{~Hz}, 2 \mathrm{CH}\right), 125.3\left(\mathrm{~d}, J_{\mathrm{P}-\mathrm{C}}=6.4 \mathrm{~Hz}\right.$, C), $124.5\left(\mathrm{~d}, J_{\mathrm{P}-\mathrm{C}}=10.0 \mathrm{~Hz}, \mathrm{CH}\right), 118.1\left(\mathrm{~d}, J_{\mathrm{P}-\mathrm{C}}=2.7 \mathrm{~Hz}, \mathrm{CH}\right)$, $116.9\left(\mathrm{~d}, J_{\mathrm{P}-\mathrm{C}}=10.9 \mathrm{~Hz}, \mathrm{CH}\right), 85.1(\mathrm{~s}, \mathrm{C}), 81.5\left(\mathrm{~d}, J_{\mathrm{P}-\mathrm{C}}=6.4 \mathrm{~Hz}\right.$, $\mathrm{CH}), 55.4\left(\mathrm{~s}, \mathrm{OCH}_{3}\right) .{ }^{31} \mathrm{P}\left\{{ }^{1} \mathrm{H}\right\} \mathrm{NMR}\left(202 \mathrm{MHz}, \mathrm{CDCl}_{3}\right): \delta 28.82$ (s). GC-MS (EI) m/z: 334 (5), 333 (19), $332(82)\left(\mathrm{M}^{+}, 331\right.$ (83), 317 (21), 316 (11), 285 (11), 284 (16), 281 (17), 271 (10), 270 (20), 269 (13), 254 (16), 253 (24), 251 (16), 240 (12), 238 (29), 226 (18), 225 (18), 223 (16), 208 (11), 207 (40), 199 (10), 196 (21), 195 (13). HRMS (ESI/Q-TOF) $m / z$ : calcd for $\mathrm{C}_{21} \mathrm{H}_{17} \mathrm{O}_{2} \mathrm{P}[\mathrm{M}$ $+\mathrm{Na}]^{+}, 355.0858$; found, 355.0860.

o-Anisyl(2-ethynylphenyl)phenylphosphine Oxide (8g). Compound 5a $(0.0486 \mathrm{~g}, 0.13 \mathrm{mmol})$ was reacted with $o-\mathrm{AnMgBr}$ (0.195 mL, $0.195 \mathrm{mmol}, 1 \mathrm{M}$ solution in THF) according to general procedure E for $30 \mathrm{~min}$ to afford $8 \mathrm{~g}(0.0397 \mathrm{~g}, 0.119 \mathrm{mmol}, 92 \%$ yield) as a yellow solid. $\mathrm{Mp}: 156.7-157.7^{\circ} \mathrm{C} . R_{f}=0.54\left(2: 1 \mathrm{CHCl}_{3} /\right.$ acetone). ${ }^{1} \mathrm{H}$ NMR (500 MHz, $\left.\mathrm{CDCl}_{3}\right): \delta 7.84-7.88(\mathrm{~m}, 2 \mathrm{H}), 7.70-$ $7.74(\mathrm{~m}, 1 \mathrm{H}), 7.61-7.66(\mathrm{~m}, 1 \mathrm{H}), 7.56-7.58(\mathrm{~m}, 1 \mathrm{H}), 7.48-7.54$ $(\mathrm{m}, 2 \mathrm{H}), 7.42-7.46(\mathrm{~m}, 3 \mathrm{H}), 7.36-7.39(\mathrm{~m}, 1 \mathrm{H}), 7.03-7.06(\mathrm{~m}$, $1 \mathrm{H}), 6.89-6.92(\mathrm{~m}, 1 \mathrm{H}), 3.54(\mathrm{~s}, 3 \mathrm{H}), 2.89(\mathrm{~s}, 1 \mathrm{H}) .{ }^{13} \mathrm{C}\left\{{ }^{1} \mathrm{H}\right\} \mathrm{NMR}$ $\left(125 \mathrm{MHz}, \mathrm{CDCl}_{3}\right): \delta 161.1\left(\mathrm{~d}, J_{\mathrm{P}-\mathrm{C}}=3.6 \mathrm{~Hz}, \mathrm{C}\right), 134.9\left(\mathrm{~d}, J_{\mathrm{P}-\mathrm{C}}=\right.$ $7.3 \mathrm{~Hz}, \mathrm{CH}), 134.4\left(\mathrm{~d}, J_{\mathrm{P}-\mathrm{C}}=9.1 \mathrm{~Hz}, \mathrm{CH}\right), 134.1\left(\mathrm{~d}, J_{\mathrm{P}-\mathrm{C}}=1.9 \mathrm{~Hz}\right.$, $\mathrm{CH}), 132.9\left(\mathrm{~d}, J_{\mathrm{P}-\mathrm{C}}=10.0 \mathrm{~Hz}, \mathrm{CH}\right), 132.2\left(\mathrm{~d}, J_{\mathrm{P}-\mathrm{C}}=10.0 \mathrm{~Hz}, 2 \mathrm{CH}\right)$, $131.4\left(\mathrm{~d}, J_{\mathrm{P}-\mathrm{C}}=1.8 \mathrm{~Hz}, \mathrm{CH}\right), 130.9\left(\mathrm{~d}, J_{\mathrm{P}-\mathrm{C}}=1.9 \mathrm{~Hz}, \mathrm{CH}\right), 128.2(\mathrm{~d}$, $\left.J_{\mathrm{P}-\mathrm{C}}=11.8 \mathrm{~Hz}, \mathrm{CH}\right), 127.9\left(\mathrm{~d}, J_{\mathrm{P}-\mathrm{C}}=12.7 \mathrm{~Hz}, 2 \mathrm{CH}\right), 124.5\left(\mathrm{~d}, J_{\mathrm{P}-\mathrm{C}}\right.$ $=6.4 \mathrm{~Hz}, \mathrm{C}), 120.8\left(\mathrm{~d}, J_{\mathrm{P}-\mathrm{C}}=11.8 \mathrm{~Hz}, \mathrm{CH}\right), 111.7\left(\mathrm{~d}, J_{\mathrm{P}-\mathrm{C}}=6.4 \mathrm{~Hz}\right.$, $\mathrm{CH}), 84.0(\mathrm{~s}, \mathrm{C}), 81.4\left(\mathrm{~d}, J_{\mathrm{P}-\mathrm{C}}=6.4 \mathrm{~Hz}, \mathrm{CH}\right), 55.3\left(\mathrm{~s}, \mathrm{CH}_{3}\right) \cdot{ }^{31} \mathrm{P}\left\{{ }^{1} \mathrm{H}\right\}$ NMR (202 MHz, CDCl $): \delta 26.19$ (s). GC-MS (EI) m/z: 333 (10), $332(42)(\mathrm{M})^{+}, 331$ (55), 254 (12), 253 (12), 252 (12), 225 (17), 196 (24), 183 (16), 178 (17), 176 (14), 165 (31), 152 (27), 151 (15), 107 (13), 101 (11), 78 (44), 77 (93), 76 (15), 75 (33), 74 (17). HRMS (ESI/Q-TOF) $m / z$ : calcd for $\mathrm{C}_{21} \mathrm{H}_{17} \mathrm{O}_{2} \mathrm{P}[\mathrm{M}+\mathrm{Na}]^{+}$, 355.0858; found, 355.0851.

2-Ethynylphenyl(9-phenanthryl)phenylphosphine Oxide (8h). Compound 5a $(0.0486 \mathrm{~g}, 0.13 \mathrm{mmol})$ was reacted with 9-phenanthryl magnesium bromide $(0.39 \mathrm{~mL}, 0.195 \mathrm{mmol}, 0.5 \mathrm{M}$ solution in THF) according to general procedure $\mathrm{E}$ for $30 \mathrm{~min}$ to afford $8 \mathrm{~h}(0.0366 \mathrm{~g}$, $0.091 \mathrm{mmol}, 70 \%$ yield) as a yellow solid. Mp: $192-193{ }^{\circ} \mathrm{C}$ (dec.). $R_{f}$ $=0.25$ (100:1 $\left.\mathrm{CHCl}_{3} / \mathrm{MTBE}\right) .{ }^{1} \mathrm{H} \operatorname{NMR}\left(500 \mathrm{MHz}, \mathrm{CDCl}_{3}\right): \delta$
8.86-8.75 (m, 3H), 7.79-7.89 (m, 3H), 7.73-7.78 (m, 3H), 7.65$7.68(\mathrm{~m}, 1 \mathrm{H}), 7.62-7.64(\mathrm{~m}, 1 \mathrm{H}), 7.57-7.60(\mathrm{~m}, 2 \mathrm{H}), 7.42-7.55$ $(\mathrm{m}, 5 \mathrm{H}), 2.84(\mathrm{~s}, 1 \mathrm{H}) .{ }^{13} \mathrm{C}\left\{{ }^{1} \mathrm{H}\right\}$ NMR $\left(125 \mathrm{MHz}, \mathrm{CDCl}_{3}\right): \delta 137.0$ $\left(\mathrm{d}, J_{\mathrm{P}-\mathrm{C}}=11.8 \mathrm{~Hz}, \mathrm{CH}\right), 135.0\left(\mathrm{~d}, J_{\mathrm{P}-\mathrm{C}}=9.1 \mathrm{~Hz}, \mathrm{CH}\right), 134.8\left(\mathrm{~d}, J_{\mathrm{P}-\mathrm{C}}\right.$ $=99.0 \mathrm{~Hz}, \mathrm{C}), 133.7\left(\mathrm{~d}, J_{\mathrm{P}-\mathrm{C}}=10.0 \mathrm{~Hz}, \mathrm{CH}\right), 132.6\left(\mathrm{~d}, J_{\mathrm{P}-\mathrm{C}}=10.0\right.$ $\mathrm{Hz}, \mathrm{CH}), 132.1\left(\mathrm{~d}, J_{\mathrm{P}-\mathrm{C}}=2.7 \mathrm{~Hz}, \mathrm{C}\right), 132.0\left(\mathrm{~d}, J_{\mathrm{P}-\mathrm{C}}=2.7 \mathrm{~Hz}, \mathrm{CH}\right)$, $131.9\left(\mathrm{~d}, J_{\mathrm{P}-\mathrm{C}}=106.3 \mathrm{~Hz}, \mathrm{C}\right), 131.7\left(\mathrm{~d}, J_{\mathrm{P}-\mathrm{C}}=1.8 \mathrm{~Hz}, \mathrm{CH}\right), 131.2(\mathrm{~d}$, $\left.J_{\mathrm{P}-\mathrm{C}}=9.1 \mathrm{~Hz}, \mathrm{C}\right), 130.5\left(\mathrm{~d}, J_{\mathrm{P}-\mathrm{C}}=9.1 \mathrm{~Hz}, \mathrm{C}\right), 130.1(\mathrm{~s}, \mathrm{CH}), 129.7$ $\left(\mathrm{d}, J_{\mathrm{P}-\mathrm{C}}=14.5 \mathrm{~Hz}, \mathrm{C}\right), 129.0(\mathrm{~s}, \mathrm{CH}), 128.6\left(\mathrm{~d}, J_{\mathrm{P}-\mathrm{C}}=11.8 \mathrm{~Hz}, \mathrm{CH}\right)$, $128.5\left(\mathrm{~d}, J_{\mathrm{P}-\mathrm{C}}=2.7 \mathrm{~Hz}, \mathrm{CH}\right), 128.4\left(\mathrm{~d}, J_{\mathrm{P}-\mathrm{C}}=12.7 \mathrm{~Hz}, \mathrm{CH}\right), 127.2$ $\left(\mathrm{d}, J_{\mathrm{P}-\mathrm{C}}=106.3 \mathrm{~Hz}, \mathrm{C}\right), 127.1\left(\mathrm{~d}, J_{\mathrm{P}-\mathrm{C}}=15.4 \mathrm{~Hz}, \mathrm{CH}\right), 126.9(\mathrm{~s}$, $\mathrm{CH}), 125.4\left(\mathrm{~d}, J_{\mathrm{P}-\mathrm{C}}=6.4 \mathrm{~Hz}, \mathrm{C}\right), 123.0(\mathrm{~s}, \mathrm{CH}), 122.7(\mathrm{~s}, \mathrm{CH}), 84.7$ $(\mathrm{s}, \mathrm{C}), 81.5\left(\mathrm{~d}, J_{\mathrm{P}-\mathrm{C}}=5.5 \mathrm{~Hz}, \mathrm{CH}\right) .{ }^{31} \mathrm{P}\left\{{ }^{1} \mathrm{H}\right\}$ NMR $(202 \mathrm{MHz}$, $\mathrm{CDCl}_{3}$ ): $\delta 31.94$ (s). HRMS (ESI/Q-TOF) $\mathrm{m} / z$ : calcd for $\mathrm{C}_{28} \mathrm{H}_{19} \mathrm{OP}$ $[2 \mathrm{M}+\mathrm{Na}]^{+}, 827.2239$; found, 827.2228.

p-Chlorophenyl(2-ethynylphenyl)phenylphosphine Oxide (8i). Compound 5a $(0.0486 \mathrm{~g}, 0.13 \mathrm{mmol})$ was reacted with $p$-Cl$\mathrm{C}_{6} \mathrm{H}_{4} \mathrm{MgBr}$ (0.195 mL, $0.195 \mathrm{mmol}, 1 \mathrm{M}$ solution in THF) according to general procedure $\mathrm{E}$ for $30 \mathrm{~min}$ to afford $8 \mathbf{i}(0.0366 \mathrm{~g}, 0.107 \mathrm{mmol}$, $82 \%$ yield $)$ as a yellow solid. $\mathrm{Mp}: 117.5-118{ }^{\circ} \mathrm{C} . R_{f}=0.55(2: 1$ $\mathrm{CHCl}_{3}$ /acetone). ${ }^{1} \mathrm{H}$ NMR (500 $\left.\mathrm{MHz}, \mathrm{CDCl}_{3}\right): \delta 7.67-7.75(\mathrm{~m}$, $5 \mathrm{H}), 7.62-7.65(\mathrm{~m}, 1 \mathrm{H}), 7.51-7.59(\mathrm{~m}, 2 \mathrm{H}), 7.42-7.49(\mathrm{~m}, 5 \mathrm{H})$, $3.02(\mathrm{~s}, 1 \mathrm{H}) .{ }^{13} \mathrm{C}\left\{{ }^{1} \mathrm{H}\right\} \operatorname{NMR}\left(125 \mathrm{MHz} \mathrm{CDCl}_{3}\right): \delta 138.4\left(\mathrm{~d}, J_{\mathrm{P}-\mathrm{C}}=\right.$ $3.6 \mathrm{~Hz}, \mathrm{C}), 135.0\left(\mathrm{~d}, J_{\mathrm{P}-\mathrm{C}}=9.1 \mathrm{~Hz}, \mathrm{CH}\right), 134.1\left(\mathrm{~d}, J_{\mathrm{P}-\mathrm{C}}=102.6 \mathrm{~Hz}\right.$, C), $133.8\left(\mathrm{~d}, J_{\mathrm{P}-\mathrm{C}}=9.1 \mathrm{~Hz}, \mathrm{CH}\right), 133.6\left(\mathrm{~d}, J_{\mathrm{P}-\mathrm{C}}=10.9 \mathrm{~Hz}, \mathrm{CH}\right)$, $132.15\left(\mathrm{~d}, J_{\mathrm{P}-\mathrm{C}}=10.0 \mathrm{~Hz}, \mathrm{CH}\right), 132.12\left(\mathrm{~d}, J_{\mathrm{P}-\mathrm{C}}=3.1 \mathrm{~Hz}, \mathrm{CH}\right), 131.9$ $\left(\mathrm{d}, J_{\mathrm{P}-\mathrm{C}}=2.5 \mathrm{~Hz}, \mathrm{CH}\right), 131.0\left(\mathrm{~d}, J_{\mathrm{P}-\mathrm{C}}=106.3 \mathrm{~Hz}, \mathrm{C}\right), 128.6\left(\mathrm{~d}, J_{\mathrm{P}-\mathrm{C}}\right.$ $=12.7 \mathrm{~Hz}, \mathrm{CH}), 128.5\left(\mathrm{~d}, J_{\mathrm{P}-\mathrm{C}}=11.8 \mathrm{~Hz}, \mathrm{CH}\right), 128.4\left(\mathrm{~d}, J_{\mathrm{P}-\mathrm{C}}=11.8\right.$ $\mathrm{Hz}, \mathrm{CH}), 125.2\left(\mathrm{~d}, J_{\mathrm{P}-\mathrm{C}}=6.4 \mathrm{~Hz}, \mathrm{C}\right), 85.3(\mathrm{~s}, \mathrm{C}), 81.5\left(\mathrm{~d}, J_{\mathrm{P}-\mathrm{C}}=6.4\right.$ $\mathrm{Hz}, \mathrm{CH}) .{ }^{31} \mathrm{P}\left\{{ }^{1} \mathrm{H}\right\}$ NMR $\left(202 \mathrm{MHz}, \mathrm{CDCl}_{3}\right): \delta 27.61$ (s). GC-MS (EI) $m / z: 338(17), 337(32), 336(61)(\mathrm{M})^{+}, 335(60), 250(21)$, 253 (19), 252 (23), 225 (14), 212 (10), 199 (12), 196 (31), 194 (13), 183 (16), 178 (18), 176 (28), 169 (13), 165 (30), 152 (37), 150 (25), 119 (12). HRMS (ESI/Q-TOF) $\mathrm{m} / \mathrm{z}$ : calcd for $\mathrm{C}_{20} \mathrm{H}_{14} \mathrm{ClOP}[\mathrm{M}+\mathrm{H}]^{+}$, 337.0544; found, 337.0537.

2-Ethynylphenyl(p-fluorophenyl)phenylphosphine Oxide (8j). Compound 5a $(0.0486 \mathrm{~g}, 0.13 \mathrm{mmol})$ was reacted with $p-\mathrm{F}$ $\mathrm{C}_{6} \mathrm{H}_{4} \mathrm{MgBr}(0.195 \mathrm{~mL}, 0.195 \mathrm{mmol}, 1 \mathrm{M}$ solution in THF) according to general procedure $\mathrm{E}$ for $30 \mathrm{~min}$ to afford $8 \mathbf{j}(0.034 \mathrm{~g}, 0.107 \mathrm{mmol}$, $82 \%$ yield $)$ as a yellow oil. $R_{f}=0.52\left(30: 5: 1 \mathrm{CHCl}_{3} / \mathrm{EtOAc} / \mathrm{MeOH}\right)$. ${ }^{1} \mathrm{H}$ NMR $\left(500 \mathrm{MHz}, \mathrm{CDCl}_{3}\right): \delta 7.68-7.77(\mathrm{~m}, 5 \mathrm{H}), 7.61-7.64(\mathrm{~m}$, $1 \mathrm{H}), 7.51-7.58(\mathrm{~m}, 2 \mathrm{H}), 7.42-7.49(\mathrm{~m}, 3 \mathrm{H}), 7.13-7.17(\mathrm{~m}, 2 \mathrm{H})$, $3.01(\mathrm{~s}, 1 \mathrm{H}) .{ }^{13} \mathrm{C}\left\{{ }^{1} \mathrm{H}\right\} \operatorname{NMR}\left(125 \mathrm{MHz}, \mathrm{CDCl}_{3}\right): \delta 165.1\left(\mathrm{dd}, J_{\mathrm{P}-\mathrm{C}}=\right.$ $\left.3.6 \mathrm{~Hz}, J_{\mathrm{F}-\mathrm{C}}=237.0 \mathrm{~Hz}, \mathrm{CF}\right), 134.9\left(\mathrm{~d}, J_{\mathrm{P}-\mathrm{C}}=8.2 \mathrm{~Hz}, \mathrm{CH}\right), 134.7$ $\left(\mathrm{dd}, J_{\mathrm{P}-\mathrm{C}}=9.1 \mathrm{~Hz}, J_{\mathrm{F}-\mathrm{C}}=11.8 \mathrm{~Hz}, 2 \mathrm{CH}\right), 134.2\left(\mathrm{~d}, J_{\mathrm{P}-\mathrm{C}}=100.8 \mathrm{~Hz}\right.$, C), $133.7\left(\mathrm{~d}, J_{\mathrm{P}-\mathrm{C}}=9.1 \mathrm{~Hz}, \mathrm{CH}\right), 132.1\left(\mathrm{~d}, J_{\mathrm{P}-\mathrm{C}}=10.0 \mathrm{~Hz}, 2 \mathrm{CH}\right)$, $132.0\left(\mathrm{~d}, J_{\mathrm{P}-\mathrm{C}}=2.7 \mathrm{~Hz}, \mathrm{CH}\right), 131.9\left(\mathrm{~d}, J_{\mathrm{P}-\mathrm{C}}=2.7 \mathrm{~Hz}, \mathrm{CH}\right), 131.8(\mathrm{~d}$, $\left.J_{\mathrm{P}-\mathrm{C}}=108.1 \mathrm{~Hz}, \mathrm{C}\right), 128.6\left(\mathrm{~d}, J_{\mathrm{P}-\mathrm{C}}=11.8 \mathrm{~Hz}, \mathrm{CH}\right), 128.4\left(\mathrm{~d}, J_{\mathrm{P}-\mathrm{C}}=\right.$ $11.8 \mathrm{~Hz}, 2 \mathrm{CH}), 128.1\left(\mathrm{dd}, J_{\mathrm{F}-\mathrm{C}}=2.7 \mathrm{~Hz}, J_{\mathrm{P}-\mathrm{C}}=108.9 \mathrm{~Hz}, \mathrm{C}\right), 125.2$ $\left(\mathrm{d}, J_{\mathrm{P}-\mathrm{C}}=6.4 \mathrm{~Hz}, \mathrm{C}\right), 115.7\left(\mathrm{dd}, J=20.9 \mathrm{~Hz}, J_{\mathrm{P}-\mathrm{C}}=13.6 \mathrm{~Hz}, \mathrm{CH}\right)$, $85.2(\mathrm{~s}, \mathrm{C}), 81.5\left(\mathrm{~d}, J_{\mathrm{P}-\mathrm{C}}=5.5 \mathrm{~Hz}, \mathrm{CH}\right) .{ }^{31} \mathrm{P}\left\{{ }^{1} \mathrm{H}\right\} \mathrm{NMR}(202 \mathrm{MHz}$, $\mathrm{CDCl}_{3}$ ): $\delta 27.61$ (s). GC-MS (EI) $\mathrm{m} / z: 321(14), 320(58)(\mathrm{M})^{+}$, 319 (68), 300 (16), 273 (15), 272 (20), 271 (19), 270 (15), 252 (11), 242 (18), 245 (18), 214 (23), 201 (20), 196 (28), 194 (20), 183 (20), 178 (11), 176 (17), 170 (19), 165 (26), 152 (26), 151 (13), 150 (16), 126 (11), 107 (17). HRMS (ESI/Q-TOF) $\mathrm{m} / z$ : calcd for $\mathrm{C}_{20} \mathrm{H}_{14} \mathrm{FOP}[\mathrm{M}+\mathrm{H}]^{+}, 321.0839$; found, 321.0841.

(2-Ethynylphenyl)phenyl[p-(N,N-dimethylamino)phenyl]phosphine Oxide (8k). Compound $5 \mathrm{a}(0.0486 \mathrm{~g}, 0.13 \mathrm{mmol})$ was reacted with $p-\mathrm{Me}_{2} \mathrm{~N}-\mathrm{C}_{6} \mathrm{H}_{4} \mathrm{MgBr}(0.39 \mathrm{~mL}, 0.195 \mathrm{mmol}, 0.5 \mathrm{M}$ solution in THF) according to general procedure $\mathrm{E}$ for $30 \mathrm{~min}$ to afford 8k (0.0269 g, $0.078 \mathrm{mmol}, 60 \%$ yield) as a yellow solid. Mp: 194.9-195.7 ${ }^{\circ} \mathrm{C} . R_{f}=0.42\left(30: 5: 1 \mathrm{CHCl}_{3} / \mathrm{EtOAc} / \mathrm{MeOH}\right) .{ }^{1} \mathrm{H}$ NMR $\left(500 \mathrm{MHz}, \mathrm{CDCl}_{3}\right): \delta 7.68-7.74(\mathrm{~m}, 3 \mathrm{H}), 7.58-7.61(\mathrm{~m}, 1 \mathrm{H})$, 7.53-7.58 (m, 2H), 7.48-7.52 (m, 1H), 7.38-7.47 (m, 4H), 6.70$6.72(\mathrm{~m}, 2 \mathrm{H}), 3.02(\mathrm{~s}, 6 \mathrm{H}), 3.02(\mathrm{~s}, 1 \mathrm{H}) .{ }^{13} \mathrm{C}\left\{{ }^{1} \mathrm{H}\right\}$ NMR $(125 \mathrm{MHz}$, $\left.\mathrm{CDCl}_{3}\right): \delta 152.3\left(\mathrm{~d}, J_{\mathrm{P}-\mathrm{C}}=2.7 \mathrm{~Hz}, \mathrm{C}\right), 135.8\left(\mathrm{~d}, J_{\mathrm{P}-\mathrm{C}}=100.8 \mathrm{~Hz}, \mathrm{C}\right)$, $134.8\left(\mathrm{~d}, J_{\mathrm{P}-\mathrm{C}}=9.1 \mathrm{~Hz}, \mathrm{CH}\right), 133.8\left(\mathrm{~d}, J_{\mathrm{P}-\mathrm{C}}=9.1 \mathrm{~Hz}, \mathrm{CH}\right), 133.6(\mathrm{~d}$, $\left.J_{\mathrm{P}-\mathrm{C}}=10.9 \mathrm{~Hz}, 2 \mathrm{CH}\right), 133.3\left(\mathrm{~d}, J_{\mathrm{P}-\mathrm{C}}=106.3 \mathrm{~Hz}, \mathrm{C}\right), 132.1\left(\mathrm{~d}, J_{\mathrm{P}-\mathrm{C}}=\right.$ $10.0 \mathrm{~Hz}, 2 \mathrm{CH}), 131.3\left(\mathrm{~d}, J_{\mathrm{P}-\mathrm{C}}=2.7 \mathrm{~Hz}, \mathrm{CH}\right), 131.2\left(\mathrm{~d}, J_{\mathrm{P}-\mathrm{C}}=1.8\right.$ 
$\mathrm{Hz}, \mathrm{CH}), 128.3\left(\mathrm{~d}, J_{\mathrm{P}-\mathrm{C}}=11.8 \mathrm{~Hz}, \mathrm{CH}\right), 128.1\left(\mathrm{~d}, J_{\mathrm{P}-\mathrm{C}}=11.8 \mathrm{~Hz}\right.$, $2 \mathrm{CH}), 125.2\left(\mathrm{~d}, J_{\mathrm{P}-\mathrm{C}}=6.4 \mathrm{~Hz}, \mathrm{C}\right), 116.3\left(\mathrm{~d}, J_{\mathrm{P}-\mathrm{C}}=118.1 \mathrm{~Hz}, \mathrm{C}\right)$, $111.1\left(\mathrm{~d}, J_{\mathrm{P}-\mathrm{C}}=12.7 \mathrm{~Hz}, 2 \mathrm{CH}\right), 84.6(\mathrm{~s}, \mathrm{C}), 81.8\left(\mathrm{~d}, J_{\mathrm{P}-\mathrm{C}}=5.5 \mathrm{~Hz}\right.$, $\mathrm{CH}), 39.9\left(\mathrm{~s}, 2 \mathrm{CH}_{3}\right) \cdot{ }^{31} \mathrm{P}\left\{{ }^{1} \mathrm{H}\right\} \mathrm{NMR}\left(202 \mathrm{MHz}, \mathrm{CDCl}_{3}\right): \delta 28.92(\mathrm{~s})$. GC-MS (EI) $m / z: 346$ (18), 345 (100) (M) ${ }^{+}, 344$ (32), 330 (15), 329 (11), 252 (16), 183 (10), 152 (12), 136 (13), 77 (12), 51 (10). HRMS (ESI/Q-TOF) $m / z$ : calcd for $\mathrm{C}_{22} \mathrm{H}_{20} \mathrm{NOP}[\mathrm{M}+\mathrm{H}]^{+}$, 346.1355; found, 346.1360 .

(2-Ethynyl-6-methylphenyl)phenyl(p-tolyl)phosphine Oxide (10a). Compound $5 \mathrm{~b}(0.0505 \mathrm{~g}, 0.13 \mathrm{mmol})$ was reacted with $p$ TolMgBr ( $0.195 \mathrm{~mL}, 0.195 \mathrm{mmol}, 1 \mathrm{M}$ solution in THF) according to general procedure $\mathrm{E}$ for $1 \mathrm{~h}$ to afford $10 \mathrm{a}(0.0283 \mathrm{~g}, 0.086 \mathrm{mmol}, 66 \%$ yield) as a yellowish oil. $R_{f}=0.15\left(200: 1 \mathrm{CHCl}_{3} / \mathrm{MTBE}\right) .{ }^{1} \mathrm{H}$ NMR $\left(500 \mathrm{MHz}, \mathrm{CDCl}_{3}\right): \delta 7.68-7.72(\mathrm{~m}, 2 \mathrm{H}), 7.59-7.63(\mathrm{~m}, 2 \mathrm{H})$, $7.50-7.54(\mathrm{~m}, 1 \mathrm{H}), 7.39-7.43(\mathrm{~m}, 3 \mathrm{H}), 7.33-7.36(\mathrm{~m}, 1 \mathrm{H}), 7.24-$ $7.27(\mathrm{~m}, 3 \mathrm{H}), 2.61(\mathrm{~s}, 1 \mathrm{H}), 2.59(\mathrm{~s}, 3 \mathrm{H}), 2.41(\mathrm{~s}, 3 \mathrm{H}) .{ }^{13} \mathrm{C}\left\{{ }^{1} \mathrm{H}\right\}$ NMR $\left(125 \mathrm{MHz}, \mathrm{CDCl}_{3}\right): \delta 145.5\left(\mathrm{~d}, J_{\mathrm{P}-\mathrm{C}}=7.3 \mathrm{~Hz}, \mathrm{C}\right), 142.3\left(\mathrm{~d}, J_{\mathrm{P}-\mathrm{C}}=\right.$ $3.6 \mathrm{~Hz}, \mathrm{C}), 134.4\left(\mathrm{~d}, J_{\mathrm{P}-\mathrm{C}}=105.4 \mathrm{~Hz}, \mathrm{C}\right), 133.6\left(\mathrm{~d}, J_{\mathrm{P}-\mathrm{C}}=9.1 \mathrm{~Hz}\right.$, $\mathrm{CH}), 133.1\left(\mathrm{~d}, J_{\mathrm{P}-\mathrm{C}}=10.0 \mathrm{~Hz}, \mathrm{CH}\right), 132.1\left(\mathrm{~d}, J_{\mathrm{P}-\mathrm{C}}=10.0 \mathrm{~Hz}, 4 \mathrm{CH}\right)$, $131.6\left(\mathrm{~d}, J_{\mathrm{P}-\mathrm{C}}=2.7 \mathrm{~Hz}, \mathrm{CH}\right), 131.1\left(\mathrm{~d}, J_{\mathrm{P}-\mathrm{C}}=2.7 \mathrm{~Hz}, \mathrm{CH}\right), 130.5(\mathrm{~d}$, $\left.J_{\mathrm{P}-\mathrm{C}}=107.2 \mathrm{~Hz}, \mathrm{C}\right), 129.1\left(\mathrm{~d}, J_{\mathrm{P}-\mathrm{C}}=12.7 \mathrm{~Hz}, 2 \mathrm{CH}\right), 129.1\left(\mathrm{~d}, J_{\mathrm{P}-\mathrm{C}}=\right.$ $11.8 \mathrm{~Hz}, 2 \mathrm{CH}), 125.7\left(\mathrm{~d}, J_{\mathrm{P}-\mathrm{C}}=9.1 \mathrm{~Hz}, \mathrm{C}\right), 84.6(\mathrm{~s}, \mathrm{C}), 82.1\left(\mathrm{~d}, J_{\mathrm{P}-\mathrm{C}}\right.$ $=6.4 \mathrm{~Hz}, \mathrm{CH}), 23.3\left(\mathrm{~d}, J_{\mathrm{P}-\mathrm{C}}=4.5 \mathrm{~Hz}, \mathrm{CH}\right), 21.6\left(\mathrm{~s}, \mathrm{CH}_{3}\right) \cdot{ }^{31} \mathrm{P}\left\{{ }^{1} \mathrm{H}\right\}$ NMR $\left(202 \mathrm{MHz}, \mathrm{CDCl}_{3}\right): \delta 30.67$ (s). GC-MS (EI) $\mathrm{m} / z: 330$ (1) $(\mathrm{M})^{+}, 329$ (1), 305 (19), 304 (100), 303 (55), 302 (18), 289 (14), 288 (22), 273 (18), 258 (11), 257 (36), 256 (16), 242 (29), 241 (24), 240 (14), 239 (22), 227 (21), 213 (19), 197 (18), 196 (18), 194 (17), 183 (49), 178 (11), 170 (12), 166 (15), 165 (67), 164 (11), 163 (14), 152 (34), 139 (17), 91 (42), 89 (25), 78 (23), 77 (70). HRMS (ESI/Q-TOF) $m / z$ : calcd for $\mathrm{C}_{22} \mathrm{H}_{19} \mathrm{OP}[\mathrm{M}+\mathrm{H}]^{+}$, 331.1246; found, 331.1236.

(2-Ethynyl-6-methylphenyl]diphenylphosphine Oxide (10d). Compound 5 b $(0.0505 \mathrm{~g}, 0.13 \mathrm{mmol})$ was reacted with $\mathrm{PhMgBr}$ (0.195 mL, $0.195 \mathrm{mmol}, 1 \mathrm{M}$ solution in THF) according to general procedure $\mathrm{E}$ for $1 \mathrm{~h}$ to afford $10 \mathrm{~d}(0.0374 \mathrm{~g}, 0.118 \mathrm{mmol}, 91 \%$ yield $)$ as a yellowish oil. $R_{f}=0.15\left(200: 1 \mathrm{CHCl}_{3} / \mathrm{MTBE}\right) .{ }^{1} \mathrm{H}$ NMR (500 $\left.\mathrm{MHz}, \mathrm{CDCl}_{3}\right): \delta 7.70-7.74(\mathrm{~m}, 4 \mathrm{H}), 7.52-7.55(\mathrm{~m}, 2 \mathrm{H}), 7.40-7.46$ (m, 5H), 7.33-7.38 (m, 1H), 7.27-7.30 (m, 1H), $2.61(\mathrm{~s}, 1 \mathrm{H}), 2.60$ $(\mathrm{s}, 3 \mathrm{H}) .{ }^{13} \mathrm{C}\left\{{ }^{1} \mathrm{H}\right\}$ NMR $\left(125 \mathrm{MHz}, \mathrm{CDCl}_{3}\right): \delta 145.6\left(\mathrm{~d}, \mathrm{~J}_{\mathrm{P}-\mathrm{C}}=7.3\right.$ $\mathrm{Hz}, \mathrm{C}), 134.4\left(\mathrm{~d}, J_{\mathrm{P}-\mathrm{C}}=1.8 \mathrm{~Hz}, \mathrm{C}\right), 133.7\left(\mathrm{~d}, J_{\mathrm{P}-\mathrm{C}}=9.1 \mathrm{~Hz}, \mathrm{CH}\right)$, $133.1\left(\mathrm{~d}, J_{\mathrm{P}-\mathrm{C}}=9.1 \mathrm{~Hz}, \mathrm{CH}\right), 132.1\left(\mathrm{~d}, J_{\mathrm{P}-\mathrm{C}}=10.9 \mathrm{~Hz}, \mathrm{CH}\right), 131.8$ $\left(\mathrm{d}, J_{\mathrm{P}-\mathrm{C}}=2.7 \mathrm{~Hz}, \mathrm{CH}\right), 131.1\left(\mathrm{~d}, J_{\mathrm{P}-\mathrm{C}}=1.8 \mathrm{~Hz}, \mathrm{CH}\right), 128.3\left(\mathrm{~d}, J_{\mathrm{P}-\mathrm{C}}\right.$ $=12.7 \mathrm{~Hz}, 2 \mathrm{CH}), 125.7\left(\mathrm{~d}, J_{\mathrm{P}-\mathrm{C}}=8.2 \mathrm{~Hz}, \mathrm{C}\right), 84.6(\mathrm{~s}, \mathrm{C}), 82.0(\mathrm{~d}$, $\left.J_{\mathrm{P}-\mathrm{C}}=6.4 \mathrm{~Hz}, \mathrm{CH}\right), 23.3\left(\mathrm{~d}, J_{\mathrm{P}-\mathrm{C}}=3.6 \mathrm{~Hz}, \mathrm{CH}_{3}\right) \cdot{ }^{31} \mathrm{P}\left\{{ }^{1} \mathrm{H}\right\} \mathrm{NMR}$ $\left(202 \mathrm{MHz}, \mathrm{CDCl}_{3}\right): \delta 30.62$ (s). GC-MS (EI) $\mathrm{m} / z: 317(5)(\mathrm{M})^{+}$, 315 (4), 291 (11), 290 (95), 289 (53), 274 (17), 259 (11), 243 (28), 243 (13), 241 (13), 239 (13), 228 (24), 212 (34), 197 (11), 194 (17), 183 (45), 165 (51), 139 (18), 77 (100). HRMS (ESI/Q-TOF) $m / z$ : calcd for $\mathrm{C}_{21} \mathrm{H}_{17} \mathrm{OP}[\mathrm{M}+\mathrm{H}]^{+}, 317.1090$; found, 317.1082.

(p-Anisyl)(2-ethynyl-6-methylphenyl]phenylphosphine Oxide (10e). Compound $5 \mathbf{b}(0.0505 \mathrm{~g}, 0.13 \mathrm{mmol})$ was reacted with $p$ $\operatorname{AnMgBr}(0.39 \mathrm{~mL}, 0.195 \mathrm{mmol}, 0.5 \mathrm{M}$ solution in THF) according to general procedure $\mathrm{E}$ for $1 \mathrm{~h}$ to afford $10 \mathrm{e}(0.0266 \mathrm{~g}, 0.0767 \mathrm{mmol}$, $59 \%$ yield $)$ as a yellowish oil. $R_{f}=0.15\left(200: 1 \mathrm{CHCl}_{3} / \mathrm{MTBE}\right) .{ }^{1} \mathrm{H}$ $\operatorname{NMR}\left(500 \mathrm{MHz}, \mathrm{CDCl}_{3}\right): \delta 7.62-7.72(\mathrm{~m}, 4 \mathrm{H}), 7.50-7.53(\mathrm{~m}, 1 \mathrm{H})$, $7.40-7.44(\mathrm{~m}, 3 \mathrm{H}), 7.33-7.38(\mathrm{~m}, 1 \mathrm{H}), 7.25-7.27(\mathrm{~m}, 1 \mathrm{H}), 6.94-$ $6.96(\mathrm{~m}, 2 \mathrm{H}), 3.85(\mathrm{~m}, 3 \mathrm{H}), 2.66(\mathrm{~s}, 1 \mathrm{H}), 2.59(\mathrm{~s}, 3 \mathrm{H}) .{ }^{13} \mathrm{C}\left\{{ }^{1} \mathrm{H}\right\}$ NMR $\left(125 \mathrm{MHz}, \mathrm{CDCl}_{3}\right): \delta 162.5\left(\mathrm{~d}, J_{\mathrm{P}-\mathrm{C}}=3.6 \mathrm{~Hz}, \mathrm{C}\right), 145.5(\mathrm{~d}$, $\left.J_{\mathrm{P}-\mathrm{C}}=7.3 \mathrm{~Hz}, \mathrm{C}\right), 134.7\left(\mathrm{~d}, J_{\mathrm{P}-\mathrm{C}}=108.1 \mathrm{~Hz}, \mathrm{C}\right), 134.1\left(\mathrm{~d}, J_{\mathrm{P}-\mathrm{C}}=\right.$ $10.9 \mathrm{~Hz}, 2 \mathrm{CH}), 133.7\left(\mathrm{~d}, J_{\mathrm{P}-\mathrm{C}}=9.1 \mathrm{~Hz}, \mathrm{CH}\right), 133.0\left(\mathrm{~d}, J_{\mathrm{P}-\mathrm{C}}=10.0\right.$ $\mathrm{Hz}, \mathrm{CH}), 132.5\left(\mathrm{~d}, J_{\mathrm{P}-\mathrm{C}}=99.9 \mathrm{~Hz}, \mathrm{C}\right), 131.9\left(\mathrm{~d}, J_{\mathrm{P}-\mathrm{C}}=10.0 \mathrm{~Hz}\right.$, $2 \mathrm{CH}), 131.6\left(\mathrm{~d}, J_{\mathrm{P}-\mathrm{C}}=2.7 \mathrm{~Hz}, \mathrm{CH}\right), 130.9\left(\mathrm{~d}, J_{\mathrm{P}-\mathrm{C}}=1.8 \mathrm{~Hz}, \mathrm{CH}\right)$, $128.3\left(\mathrm{~d}, J_{\mathrm{P}-\mathrm{C}}=11.8 \mathrm{~Hz}, 2 \mathrm{CH}\right), 125.6\left(\mathrm{~d}, J_{\mathrm{P}-\mathrm{C}}=6.4 \mathrm{~Hz}, \mathrm{C}\right), 113.2(\mathrm{~d}$, $\left.J_{\mathrm{P}-\mathrm{C}}=13.6 \mathrm{~Hz}, 2 \mathrm{CH}\right), 84.4(\mathrm{~s}, \mathrm{C}), 82.2\left(\mathrm{~d}, J_{\mathrm{P}-\mathrm{C}}=6.4 \mathrm{~Hz}, \mathrm{CH}\right), 56.3$ $\left(\mathrm{s}, \mathrm{CH}_{3}\right), 23.3\left(\mathrm{~d}, J_{\mathrm{P}-\mathrm{C}}=3.6 \mathrm{~Hz}, \mathrm{CH}_{3}\right) .{ }^{31} \mathrm{P}\left\{{ }^{1} \mathrm{H}\right\} \mathrm{NMR}(202 \mathrm{MHz}$, $\mathrm{CDCl}_{3}$ ): $\delta 30.21(\mathrm{~s})$. HRMS (ESI/Q-TOF) $\mathrm{m} / z$ : calcd for $\mathrm{C}_{22} \mathrm{H}_{19} \mathrm{O}_{2} \mathrm{P}[\mathrm{M}+\mathrm{H}]^{+}$, 347.1195; found, 347.1205.

(2-Ethynyl-6-methylphenyl)(4-chlorophenyl)phenylphosphine Oxide (10i). Compound $5 \mathbf{b}(0.0505 \mathrm{~g}, 0.13 \mathrm{mmol})$ was reacted with $p$ - $\mathrm{Cl}-\mathrm{C}_{6} \mathrm{H}_{4} \mathrm{MgBr}(0.195 \mathrm{~mL}, 0.195 \mathrm{mmol}, 1 \mathrm{M}$ solution in THF) according to general procedure $\mathrm{E}$ for $1 \mathrm{~h}$ to afford $10 \mathrm{i}(0.0296 \mathrm{~g}$, $0.0845 \mathrm{mmol}, 65 \%$ yield $)$ as a yellowish oil. $R_{f}=0.4\left(30: 5: 1 \mathrm{CHCl}_{3} /\right.$ AcOEt/MeOH). ${ }^{1} \mathrm{H}$ NMR $\left(500 \mathrm{MHz}, \mathrm{CDCl}_{3}\right): \delta 7.69-7.73(\mathrm{~m}$, $2 \mathrm{H})$, 7.63-7.67 (m, 2H), 7.54-7.57 (m, $1 \mathrm{H}), 7.40-7.47(\mathrm{~m}, 5 \mathrm{H})$, 7.36-7.38 (m, 1H), 7.27-7.29 (m, 1H), $2.67(\mathrm{~m}, 1 \mathrm{H}), 2.59(\mathrm{~s}, 3 \mathrm{H})$. ${ }^{13} \mathrm{C}\left\{{ }^{1} \mathrm{H}\right\}$ NMR $\left(125 \mathrm{MHz}, \mathrm{CDCl}_{3}\right): \delta 145.6\left(\mathrm{~d}, J_{\mathrm{P}-\mathrm{C}}=8.2 \mathrm{~Hz}, \mathrm{C}\right)$, $138.3\left(\mathrm{~d}, J_{\mathrm{P}-\mathrm{C}}=3.6 \mathrm{~Hz}, \mathrm{C}\right), 133.7\left(\mathrm{~d}, J_{\mathrm{P}-\mathrm{C}}=9.1 \mathrm{~Hz}, \mathrm{CH}\right), 133.6(\mathrm{~d}$, $\left.J_{\mathrm{P}-\mathrm{C}}=102.6 \mathrm{~Hz}, \mathrm{C}\right), 133.5\left(\mathrm{~d}, J_{\mathrm{P}-\mathrm{C}}=10.9 \mathrm{~Hz}, 2 \mathrm{CH}\right), 133.2\left(\mathrm{~d}, J_{\mathrm{P}-\mathrm{C}}=\right.$ $10.0 \mathrm{~Hz}, \mathrm{CH}), 132.9\left(\mathrm{~d}, J_{\mathrm{P}-\mathrm{C}}=106.3 \mathrm{~Hz}, \mathrm{C}\right), 131.9\left(\mathrm{~d}, J_{\mathrm{P}-\mathrm{C}}=2.7 \mathrm{~Hz}\right.$, $\mathrm{CH}), 131.8\left(\mathrm{~d}, J_{\mathrm{P}-\mathrm{C}}=10.0 \mathrm{~Hz}, 2 \mathrm{CH}\right), 131.3\left(\mathrm{~d}, J_{\mathrm{P}-\mathrm{C}}=1.8 \mathrm{~Hz}, \mathrm{CH}\right)$, $128.6\left(\mathrm{~d}, J_{\mathrm{P}-\mathrm{C}}=12.7 \mathrm{~Hz}, 2 \mathrm{CH}\right), 128.5\left(\mathrm{~d}, J_{\mathrm{P}-\mathrm{C}}=12.7 \mathrm{~Hz}, 2 \mathrm{CH}\right)$, $125.6\left(\mathrm{~d}, J_{\mathrm{P}-\mathrm{C}}=8.2 \mathrm{~Hz}, \mathrm{C}\right), 85.1(\mathrm{~s}, \mathrm{C}), 82.1\left(\mathrm{~d}, J_{\mathrm{P}-\mathrm{C}}=6.4 \mathrm{~Hz}, \mathrm{CH}\right)$, $23.3\left(\mathrm{~d}, J_{\mathrm{P}-\mathrm{C}}=3.6 \mathrm{~Hz}, \mathrm{CH}_{3}\right) .{ }^{31} \mathrm{P}\left\{{ }^{1} \mathrm{H}\right\} \mathrm{NMR}\left(202 \mathrm{MHz}, \mathrm{CDCl}_{3}\right): \delta$ 29.69 (s). HRMS (ESI/Q-TOF) $m / z$ : calcd for $\mathrm{C}_{21} \mathrm{H}_{16} \mathrm{ClOP}[\mathrm{M}+$ $\mathrm{H}]^{+}, 351.0700$; found, 351.0694 .

(2-Ethynyl-6-methylphenyl) [ $\mathrm{p}$-( $\mathrm{N}, \mathrm{N}$-dimethylamino)phenyl]phenylphosphine Oxide (10k). Compound $5 \mathbf{b}(0.0505 \mathrm{~g}, 0.13$ mmol) was reacted with $p-\mathrm{Me}_{2} \mathrm{~N}-\mathrm{C}_{6} \mathrm{H}_{4} \mathrm{MgBr}(0.39 \mathrm{~mL}, 0.195 \mathrm{mmol}$, $0.5 \mathrm{M}$ solution in THF) according to general procedure $\mathrm{E}$ for $1 \mathrm{~h}$ to afford 10k $(0.0327 \mathrm{~g}, 0.091 \mathrm{mmol}, 70 \%$ yield $)$ as a brownish waxy solid. Mp: $140.5-144.5{ }^{\circ} \mathrm{C} . R_{f}=0.21\left(10: 1 \mathrm{CHCl}_{3} / \mathrm{MTBE}\right) .{ }^{1} \mathrm{H}$ $\operatorname{NMR}\left(500 \mathrm{MHz}, \mathrm{CDCl}_{3}\right): \delta 7.66-7.70(\mathrm{~m}, 2 \mathrm{H}), 7.51-7.55(\mathrm{~m}, 2 \mathrm{H})$, 7.47-7.50 (m, 1H), 7.38-7.41 (m, 3H), 7.30-7.33 (m, $1 \mathrm{H}), 7.23-$ $7.30(\mathrm{~m}, 1 \mathrm{H}), 6.69-6.71(\mathrm{~m}, 2 \mathrm{H}), 3.01(\mathrm{~s}, 6 \mathrm{H}), 2.67(\mathrm{~s}, 1 \mathrm{H}), 2.60$ $(\mathrm{m}, 3 \mathrm{H}) .{ }^{13} \mathrm{C}\left\{{ }^{1} \mathrm{H}\right\}$ NMR $\left(125 \mathrm{MHz}, \mathrm{CDCl}_{3}\right): \delta 152.5\left(\mathrm{~d}, \mathrm{~J}_{\mathrm{P}-\mathrm{C}}=2.7\right.$ $\mathrm{Hz}, \mathrm{C}), 145.3\left(\mathrm{~d}, J_{\mathrm{P}-\mathrm{C}}=7.3 \mathrm{~Hz}, \mathrm{C}\right), 135.6\left(\mathrm{~d}, J_{\mathrm{P}-\mathrm{C}}=105.4 \mathrm{~Hz}, \mathrm{C}\right)$, $133.6\left(\mathrm{~d}, J_{\mathrm{P}-\mathrm{C}}=9.1 \mathrm{~Hz}, \mathrm{CH}\right), 133.5\left(\mathrm{~d}, J_{\mathrm{P}-\mathrm{C}}=11.8 \mathrm{~Hz}, 2 \mathrm{CH}\right), 133.2$ $\left(\mathrm{d}, J_{\mathrm{P}-\mathrm{C}}=99.0 \mathrm{~Hz}, \mathrm{C}\right), 132.9\left(\mathrm{~d}, J_{\mathrm{P}-\mathrm{C}}=10.0 \mathrm{~Hz}, \mathrm{CH}\right), 131.9\left(\mathrm{~d}, J_{\mathrm{P}-\mathrm{C}}\right.$ $=10.0 \mathrm{~Hz}, 2 \mathrm{CH}), 131.3\left(\mathrm{~d}, J_{\mathrm{P}-\mathrm{C}}=3.6 \mathrm{~Hz}, \mathrm{CH}\right), 130.6\left(\mathrm{~d}, J_{\mathrm{P}-\mathrm{C}}=1.8\right.$ $\mathrm{Hz}, \mathrm{CH}), 128.6\left(\mathrm{~d}, J_{\mathrm{P}-\mathrm{C}}=101.7 \mathrm{~Hz}, \mathrm{C}\right), 128.1\left(\mathrm{~d}, J_{\mathrm{P}-\mathrm{C}}=12.7 \mathrm{~Hz}\right.$, $2 \mathrm{CH}), 123.1\left(\mathrm{~d}, J_{\mathrm{P}-\mathrm{C}}=8.2 \mathrm{~Hz}, \mathrm{C}\right), 111.2\left(\mathrm{~d}, J_{\mathrm{P}-\mathrm{C}}=12.7 \mathrm{~Hz}, 2 \mathrm{CH}\right)$, $84.1(\mathrm{~s}, \mathrm{C}), 82.3\left(\mathrm{~d}, J_{\mathrm{P}-\mathrm{C}}=6.4 \mathrm{~Hz}, \mathrm{CH}\right), 39.9\left(\mathrm{~s}, 2 \mathrm{CH}_{3}\right), 23.3\left(\mathrm{~d}, J_{\mathrm{P}-\mathrm{C}}\right.$ $\left.=4.5 \mathrm{~Hz}, \mathrm{CH}_{3}\right) \cdot{ }^{31} \mathrm{P}\left\{{ }^{1} \mathrm{H}\right\} \operatorname{NMR}\left(202 \mathrm{MHz}, \mathrm{CDCl}_{3}\right): \delta 31.06(\mathrm{~s})$. HRMS (ESI/Q-TOF) $m / z$ : calcd for $\mathrm{C}_{23} \mathrm{H}_{22} \mathrm{NOP}[\mathrm{M}+\mathrm{H}]^{+}$, 360.1512; found, 360.1515 .

(2-Ethynyl-5-chlorophenyl)phenyl(p-tolyl)phosphine Oxide (11a). Compound 5c $(0.053 \mathrm{~g}, 0.13 \mathrm{mmol})$ was reacted with $p$ TolMgBr ( $0.195 \mathrm{~mL}, 0.195 \mathrm{mmol}, 1 \mathrm{M}$ solution in THF) according to general procedure $\mathrm{E}$ for $1 \mathrm{~h}$ to afford $11 \mathrm{a}(0.0301 \mathrm{~g}, 0.086 \mathrm{mmol}, 66 \%$ yield) as an orange solid. Mp: $160.4-161.8{ }^{\circ} \mathrm{C} . R_{f}=0.45$ (30:5:1 $\left.\mathrm{CHCl}_{3} / \mathrm{AcOEt} / \mathrm{MeOH}\right) .{ }^{1} \mathrm{H}$ NMR $\left(500 \mathrm{MHz}, \mathrm{CDCl}_{3}\right): \delta 7.71-7.75$ (m, 3H), 7.61-7.66 (m, 2H), 7.52-7.56 (m, 2H), 7.45-7.49 (m, $3 \mathrm{H}), 7.27-7.30(\mathrm{~m}, 2 \mathrm{H}), 3.02(\mathrm{~m}, 1 \mathrm{H}), 2.43(\mathrm{~m}, 3 \mathrm{H}) .{ }^{13} \mathrm{C}\left\{{ }^{1} \mathrm{H}\right\} \mathrm{NMR}$ $\left(125 \mathrm{MHz}, \mathrm{CDCl}_{3}\right): \delta 142.8\left(\mathrm{~d}, J_{\mathrm{P}-\mathrm{C}}=2.7 \mathrm{~Hz}, \mathrm{C}\right), 137.3\left(\mathrm{~d}, J_{\mathrm{P}-\mathrm{C}}=\right.$ $97.2 \mathrm{~Hz}, \mathrm{C}), 136.1\left(\mathrm{~d}, J_{\mathrm{P}-\mathrm{C}}=10.0 \mathrm{~Hz}, \mathrm{CH}\right), 136.1\left(\mathrm{~d}, J_{\mathrm{P}-\mathrm{C}}=14.5 \mathrm{~Hz}\right.$, C), $133.7\left(\mathrm{~d}, J_{\mathrm{P}-\mathrm{C}}=10.0 \mathrm{~Hz}, \mathrm{CH}\right), 132.3\left(\mathrm{~d}, J_{\mathrm{P}-\mathrm{C}}=10.0 \mathrm{~Hz}, 2 \mathrm{CH}\right)$, $132.2\left(\mathrm{~d}, J_{\mathrm{P}-\mathrm{C}}=10.0 \mathrm{~Hz}, 2 \mathrm{CH}\right), 132.1\left(\mathrm{~d}, J_{\mathrm{P}-\mathrm{C}}=2.7 \mathrm{~Hz}, \mathrm{CH}\right), 131.9$ $\left(\mathrm{d}, J_{\mathrm{P}-\mathrm{C}}=108.9 \mathrm{~Hz}, \mathrm{C}\right), 131.8\left(\mathrm{~d}, J_{\mathrm{P}-\mathrm{C}}=2.7 \mathrm{~Hz}, \mathrm{CH}\right), 129.3\left(\mathrm{~d}, J_{\mathrm{P}-\mathrm{C}}\right.$ $=12.7 \mathrm{~Hz}, 2 \mathrm{CH}), 128.4\left(\mathrm{~d}, J_{\mathrm{P}-\mathrm{C}}=12.7 \mathrm{~Hz}, 2 \mathrm{CH}\right), 128.1\left(\mathrm{~d}, J_{\mathrm{P}-\mathrm{C}}=\right.$ $109.9 \mathrm{~Hz}, \mathrm{C}), 123.5\left(\mathrm{~d}, J_{\mathrm{P}-\mathrm{C}}=6.4 \mathrm{~Hz}, \mathrm{C}\right), 85.9(\mathrm{~s}, \mathrm{C}), 80.8\left(\mathrm{~d}, J_{\mathrm{P}-\mathrm{C}}=\right.$ $5.5 \mathrm{~Hz}, \mathrm{CH}) .{ }^{31} \mathrm{P}\left\{{ }^{1} \mathrm{H}\right\}$ NMR $\left(202 \mathrm{MHz}, \mathrm{CDCl}_{3}\right): \delta 27.47$ (s). GCMS (EI) $m / z: 353(8), 352(31), 351(52), 350(95)\left(\mathrm{M}^{+}, 349\right.$ (91), 331 (8), 302 (12), 273 (27), 268 (25), 258 (13), 256 (12), 252 (23), 244 (17), 230 (14), 215 (22), 212 (14), 210 (13), 199 (13), 196 (15), 194 (22), 189 (29), 176 (25), 165 (31). HRMS (ESI/Q-TOF) $m / z$ : calcd for $\mathrm{C}_{21} \mathrm{H}_{16} \mathrm{ClOP}[\mathrm{M}+\mathrm{H}]^{+}$, 351.0700; found, 351.0693.

(2-Ethynyl-5-chlorophenyl]diphenylphosphine Oxide (11d). Compound 5c $(0.053 \mathrm{~g}, 0.13 \mathrm{mmol})$ was reacted with $\mathrm{PhMgBr}$ $(0.195 \mathrm{~mL}, 0.195 \mathrm{mmol}, 1 \mathrm{M}$ solution in THF) according to general procedure $\mathrm{E}$ for $1 \mathrm{~h}$ to afford $11 \mathrm{~d}(0.032 \mathrm{~g}, 0.095 \mathrm{mmol}, 73 \%$ yield $)$ as an yellow oil. $R_{f}=0.48\left(30: 5: 1 \mathrm{CHCl}_{3} / \mathrm{AcOEt} / \mathrm{MeOH}\right) .{ }^{1} \mathrm{H}$ NMR $\left(500 \mathrm{MHz}, \mathrm{CDCl}_{3}\right): \delta$ 7.73-7.77 (m, 5H), 7.53-7.59 (m, 3H), 7.46-7.50 (m, 5H), $3.01(\mathrm{~s}, 1 \mathrm{H}) .{ }^{13} \mathrm{C}\left\{{ }^{1} \mathrm{H}\right\}$ NMR $\left(125 \mathrm{MHz}, \mathrm{CDCl}_{3}\right)$ : $\delta 136.9\left(\mathrm{~d}, J_{\mathrm{P}-\mathrm{C}}=98.1 \mathrm{~Hz}, \mathrm{C}\right), 136.1\left(\mathrm{~d}, J_{\mathrm{P}-\mathrm{C}}=10.0 \mathrm{~Hz}, \mathrm{CH}\right), 135.2$ $\left(\mathrm{d}, J_{\mathrm{P}-\mathrm{C}}=14.5 \mathrm{~Hz}, \mathrm{C}\right), 133.7\left(\mathrm{~d}, J_{\mathrm{P}-\mathrm{C}}=9.1 \mathrm{~Hz}, \mathrm{CH}\right), 132.2\left(\mathrm{~d}, J_{\mathrm{P}-\mathrm{C}}=\right.$ $10.0 \mathrm{~Hz}, 4 \mathrm{CH}), 132.17\left(\mathrm{~d}, J_{\mathrm{P}-\mathrm{C}}=4.5 \mathrm{~Hz}, 2 \mathrm{CH}\right), 131.9\left(\mathrm{~d}, J_{\mathrm{P}-\mathrm{C}}=2.7\right.$ $\mathrm{Hz}, \mathrm{CH}), 131.4\left(\mathrm{~d}, J_{\mathrm{P}-\mathrm{C}}=107.2 \mathrm{~Hz}, 2 \mathrm{C}\right), 128.5\left(\mathrm{~d}, J_{\mathrm{P}-\mathrm{C}}=12.7 \mathrm{~Hz}\right.$, $4 \mathrm{CH}), 123.5\left(\mathrm{~d}, J_{\mathrm{P}-\mathrm{C}}=6.4 \mathrm{~Hz}, \mathrm{C}\right), 84.9(\mathrm{~s}, \mathrm{C}), 80.7\left(\mathrm{~d}, J_{\mathrm{P}-\mathrm{C}}=5.5 \mathrm{~Hz}\right.$ $\mathrm{CH}) .{ }^{31} \mathrm{P}\left\{{ }^{1} \mathrm{H}\right\}$ NMR $\left(202 \mathrm{MHz}, \mathrm{CDCl}_{3}\right): \delta 27.46$ (s). GC-MS (EI) $m / z: 338$ (14), 337 (33), $336(46)(\mathrm{M})^{+}, 335$ (41), 259 (13), 254 
(15), 252 (12), 230 (11), 207 (17), 199 (13), 196 (14), 194 (12), 176 (12), 154 (17), 152 (14), 135 (14), 100 (11), 99 (37), 98 (10), 78 (59), 77 (100), 76 (15), 75 (22), 74 (23), 51 (89). HRMS (ESI/ Q-TOF) $m / z$ : calcd for $\mathrm{C}_{20} \mathrm{H}_{14} \mathrm{ClOP}[\mathrm{M}+\mathrm{H}]^{+}, 337.0544$; found, 337.0543.

(p-Anisyl)(2-ethynyl-5-chlorophenyl]phenylphosphine Oxide (11e). Compound 5c $(0.053 \mathrm{~g}, 0.13 \mathrm{mmol})$ was reacted with $p$ AnMgBr $(0.39 \mathrm{~mL}, 0.195 \mathrm{mmol}, 0.5 \mathrm{M}$ solution in THF) according to general procedure $\mathrm{E}$ for $1 \mathrm{~h}$ to afford $11 \mathrm{e}(0.0276 \mathrm{~g}, 0.0754 \mathrm{mmol}$, $58 \%$ yield $)$ as an orange oil. $R_{f}=0.54\left(30: 5: 1 \mathrm{CHCl}_{3} / \mathrm{AcOEt} /\right.$ $\mathrm{MeOH}) .{ }^{1} \mathrm{H}$ NMR (500 MHz, $\mathrm{CDCl}_{3}$ ): $\delta 7.65-7.77$ (m, 5H), 7.52$7.56(\mathrm{~m}, 2 \mathrm{H}), 7.45-7.48(\mathrm{~m}, 3 \mathrm{H}), 6.69-6.99(\mathrm{~m}, 2 \mathrm{H}), 3.86(\mathrm{~s}, 3 \mathrm{H})$, $3.03(\mathrm{~s}, 1 \mathrm{H}) \cdot{ }^{13} \mathrm{C}\left\{{ }^{1} \mathrm{H}\right\} \mathrm{NMR}\left(125 \mathrm{MHz}, \mathrm{CDCl}_{3}\right): \delta 162.7\left(\mathrm{~d}, J_{\mathrm{P}-\mathrm{C}}=\right.$ $3.6 \mathrm{~Hz}, \mathrm{C}), 137.5\left(\mathrm{~d}, J_{\mathrm{P}-\mathrm{C}}=98.1 \mathrm{~Hz}, \mathrm{C}\right), 136.2\left(\mathrm{~d}, J_{\mathrm{P}-\mathrm{C}}=10.0 \mathrm{~Hz}\right.$, $\mathrm{CH}), 135.2\left(\mathrm{~d}, J_{\mathrm{P}-\mathrm{C}}=14.5 \mathrm{~Hz}, \mathrm{C}\right), 134.2\left(\mathrm{~d}, J_{\mathrm{P}-\mathrm{C}}=11.8 \mathrm{~Hz}, 2 \mathrm{CH}\right)$, $133.7\left(\mathrm{~d}, J_{\mathrm{P}-\mathrm{C}}=10.0 \mathrm{~Hz}, \mathrm{CH}\right), 132.2\left(\mathrm{~d}, J_{\mathrm{P}-\mathrm{C}}=10.9 \mathrm{~Hz}, 2 \mathrm{CH}\right), 132.0$ $\left(\mathrm{d}, J_{\mathrm{P}-\mathrm{C}}=3.6 \mathrm{~Hz}, \mathrm{CH}\right), 131.9\left(\mathrm{~d}, J_{\mathrm{P}-\mathrm{C}}=108.1 \mathrm{~Hz}, \mathrm{C}\right), 131.8\left(\mathrm{~d}, J_{\mathrm{P}-\mathrm{C}}\right.$ $=2.7 \mathrm{~Hz}, \mathrm{CH}), 128.4\left(\mathrm{~d}, J_{\mathrm{P}-\mathrm{C}}=12.7 \mathrm{~Hz}, 2 \mathrm{CH}\right), 123.4\left(\mathrm{~d}, J_{\mathrm{P}-\mathrm{C}}=6.4\right.$ $\mathrm{Hz}, \mathrm{C}), 122.4\left(\mathrm{~d}, J_{\mathrm{P}-\mathrm{C}}=113.5 \mathrm{~Hz}, \mathrm{C}\right), 114.1\left(\mathrm{~d}, J_{\mathrm{P}-\mathrm{C}}=13.6 \mathrm{~Hz}\right.$, $2 \mathrm{CH}), 85.6(\mathrm{~s}, \mathrm{C}), 80.8\left(\mathrm{~d}, J_{\mathrm{P}-\mathrm{C}}=4.5 \mathrm{~Hz}, \mathrm{CH}\right), 55.4\left(\mathrm{~s}, \mathrm{OCH}_{3}\right)$. ${ }^{31} \mathrm{P}\left\{{ }^{1} \mathrm{H}\right\}$ NMR (202 MHz, $\left.\mathrm{CDCl}_{3}\right): \delta 27.26(\mathrm{~s})$. GC-MS (EI) $\mathrm{m} / z$ : 368 (27), 367 (48), $366(100)(\mathrm{M})^{+}, 365$ (76), 353 (13), 351 (23), 289 (24), 283 (11), 252 (10), 245 (12), 239 (15), 217 (12), 199 (25), 182 (11), 176 (19), 170 (10), 152 (11), 151 (10), 141 (14) 139 (11), 138 (10), 123 (37), 110 (11), 108 (24), 107 (13). HRMS (ESI/Q-TOF) $\mathrm{m} / z$ : calcd for $\mathrm{C}_{21} \mathrm{H}_{16} \mathrm{ClO}_{2} \mathrm{P}[\mathrm{M}+\mathrm{H}]^{+}, 367.0649$; found, 367.0642 .

(4-Chlorophenyl)(2-ethynyl-5-chlorophenyl]phenylphosphine Oxide (11i). Compound $5 \mathrm{c}(0.053 \mathrm{~g}, 0.13 \mathrm{mmol})$ was reacted with $p$ $\mathrm{Cl}-\mathrm{C}_{6} \mathrm{H}_{4} \mathrm{MgBr}(0.195 \mathrm{~mL}, 0.195 \mathrm{mmol}, 1 \mathrm{M}$ solution in THF $)$ according to general procedure $\mathrm{E}$ for $1 \mathrm{~h}$ to afford $11 \mathrm{i}(0.0289 \mathrm{~g}$, $0.078 \mathrm{mmol}, 60 \%$ yield $)$ as an yellow oil. $R_{f}=0.47\left(30: 5: 1 \mathrm{CHCl}_{3} /\right.$ AcOEt/MeOH). ${ }^{1} \mathrm{H}$ NMR $\left(500 \mathrm{MHz}, \mathrm{CDCl}_{3}\right): \delta 7.67-7.77(\mathrm{~m}$, $5 \mathrm{H}), 7.54-7.60(\mathrm{~m}, 2 \mathrm{H}), 7.45-7.51(\mathrm{~m}, 5 \mathrm{H}), 3.04(\mathrm{~s}, 1 \mathrm{H}) .{ }^{13} \mathrm{C}\left\{{ }^{1} \mathrm{H}\right\}$ NMR $\left(125 \mathrm{MHz}, \mathrm{CDCl}_{3}\right): \delta 138.7\left(\mathrm{~d}, J_{\mathrm{P}-\mathrm{C}}=3.6 \mathrm{~Hz}, \mathrm{C}\right), 136.3(\mathrm{~d}$, $\left.J_{\mathrm{P}-\mathrm{C}}=10.0 \mathrm{~Hz}, 2 \mathrm{CH}\right), 135.4\left(\mathrm{~d}, J_{\mathrm{P}-\mathrm{C}}=15.4 \mathrm{~Hz}, \mathrm{C}\right), 133.7\left(\mathrm{~d}, J_{\mathrm{P}-\mathrm{C}}=\right.$ $10.0 \mathrm{~Hz}, \mathrm{CH}), 133.6\left(\mathrm{~d}, J_{\mathrm{P}-\mathrm{C}}=10.9 \mathrm{~Hz}, 2 \mathrm{CH}\right), 132.4\left(\mathrm{~d}, J_{\mathrm{P}-\mathrm{C}}=1.8\right.$ $\mathrm{Hz}, \mathrm{CH}), 132.1\left(\mathrm{~d}, J_{\mathrm{P}-\mathrm{C}}=1.8 \mathrm{~Hz}, \mathrm{CH}\right), 132.1\left(\mathrm{~d}, J_{\mathrm{P}-\mathrm{C}}=10.0 \mathrm{~Hz}\right.$, $2 \mathrm{CH}), 128.8\left(\mathrm{~d}, J_{\mathrm{P}-\mathrm{C}}=13.6 \mathrm{~Hz}, 2 \mathrm{CH}\right), 128.6\left(\mathrm{~d}, J_{\mathrm{P}-\mathrm{C}}=12.7 \mathrm{~Hz}\right.$, $2 \mathrm{CH}), 123.3\left(\mathrm{~d}, J_{\mathrm{P}-\mathrm{C}}=6.4 \mathrm{~Hz}, \mathrm{C}\right), 86.2(\mathrm{~s}, \mathrm{C}), 80.7\left(\mathrm{~d}, J_{\mathrm{P}-\mathrm{C}}=5.5 \mathrm{~Hz}\right.$, $\mathrm{CH}) .{ }^{31} \mathrm{P}\left\{{ }^{1} \mathrm{H}\right\}$ NMR $\left(202 \mathrm{MHz}, \mathrm{CDCl}_{3}\right): \delta 26.56(\mathrm{~s})$. GC-MS (EI) $m / z: 373$ (13), 372 (18), 371 (59), 370 (100) (M) ${ }^{+}, 369$ (24), 355 (22), 354 (11), 334 (17), 325 (14), 297 (11), 288 (14), 283 (14), 266 (18), 264 (25), 252 (43), 243 (13), 241 (18), 235 (10), 230 (28), 216 (14), 207 (21), 199 (13), 197 (13), 196 (16), 177 (18), 174 (13), 175 (12), 174 (22), 166 (12), 159 (12), 152 (12), 137 (11), 136 (17), 133 (41), 126 (10), 119 (13), 113 (14), 112 (36), 111 (34), 108 (11), 107 (30), 99 (37), 98 (17), 78 (12), 77 (41), 76 (24), 75 (69), 74 (35), 72 (21). HRMS (ESI/Q-TOF) $\mathrm{m} / z$ : calcd for $\mathrm{C}_{20} \mathrm{H}_{13} \mathrm{Cl}_{2} \mathrm{OP}[\mathrm{M}+\mathrm{H}]^{+}$, 371.0154; found, 371.0145.

(2-Ethynyl-5-chlorophenyl) [p-(N,N-dimethylamino)phenyl]phenyl Phosphine Oxide (11 k). Compound 5c $(0.053 \mathrm{~g}, 0.13 \mathrm{mmol})$ was reacted with $p-\mathrm{Me}_{2} \mathrm{~N}-\mathrm{C}_{6} \mathrm{H}_{4} \mathrm{MgBr}(0.39 \mathrm{~mL}, 0.195 \mathrm{mmol}, 0.5 \mathrm{M}$ solution in THF) according to general procedure $\mathrm{E}$ for $1 \mathrm{~h}$ to afford $11 \mathrm{k}(0.0296 \mathrm{~g}, 0.078 \mathrm{mmol}, 60 \%$ yield $)$ as an orange oil. $R_{f}=0.21$ (10:1 $\left.\mathrm{CHCl}_{3} / \mathrm{MTBE}\right) .{ }^{1} \mathrm{H}$ NMR $\left(500 \mathrm{MHz}, \mathrm{CDCl}_{3}\right): \delta 7.77-7.78$ $(\mathrm{m}, 1 \mathrm{H}), 7.70-7.74(\mathrm{~m}, 2 \mathrm{H}), 7.50-7.58(\mathrm{~m}, 4 \mathrm{H}), 7.43-7.46(\mathrm{~m}$, $3 \mathrm{H}), 6.72-6.74(\mathrm{~m}, 2 \mathrm{H}), 3.03(\mathrm{~s}, 7 \mathrm{H}) .{ }^{13} \mathrm{C}\left\{{ }^{1} \mathrm{H}\right\}$ NMR $(125 \mathrm{MHz}$, $\left.\mathrm{CDCl}_{3}\right): \delta 152.4\left(\mathrm{~d}, J_{\mathrm{P}-\mathrm{C}}=1.8 \mathrm{~Hz}, \mathrm{C}\right), 138.1\left(\mathrm{~d}, J_{\mathrm{P}-\mathrm{C}}=92.6 \mathrm{~Hz}, \mathrm{C}\right)$, $135.9\left(\mathrm{~d}, J_{\mathrm{P}-\mathrm{C}}=10.5 \mathrm{~Hz}, \mathrm{CH}\right), 134.9\left(\mathrm{~d}, J_{\mathrm{P}-\mathrm{C}}=14.5 \mathrm{~Hz}, \mathrm{CH}\right), 133.6$ $\left(\mathrm{d}, J_{\mathrm{P}-\mathrm{C}}=10.0 \mathrm{~Hz}, \mathrm{CH}\right), 133.5\left(\mathrm{~d}, J_{\mathrm{P}-\mathrm{C}}=10.9 \mathrm{~Hz}, 2 \mathrm{CH}\right), 132.6(\mathrm{~d}$, $\left.J_{\mathrm{P}-\mathrm{C}}=107.2 \mathrm{~Hz}, \mathrm{C}\right), 132.1\left(\mathrm{~d}, J_{\mathrm{P}-\mathrm{C}}=10.0 \mathrm{~Hz}, 2 \mathrm{CH}\right), 131.7\left(\mathrm{~d}, J_{\mathrm{P}-\mathrm{C}}=\right.$ $2.7 \mathrm{~Hz}, \mathrm{CH}), 131.4\left(\mathrm{~d}, J_{\mathrm{P}-\mathrm{C}}=1.8 \mathrm{~Hz}, \mathrm{CH}\right), 128.2\left(\mathrm{~d}, J_{\mathrm{P}-\mathrm{C}}=12.7 \mathrm{~Hz}\right.$, $2 \mathrm{CH}), 123.1\left(\mathrm{~d}, J_{\mathrm{P}-\mathrm{C}}=5.5 \mathrm{~Hz}, \mathrm{C}\right), 115.2\left(\mathrm{~d}, J_{\mathrm{P}-\mathrm{C}}=118.0 \mathrm{~Hz}, \mathrm{C}\right)$, $111.1\left(\mathrm{~d}, J_{\mathrm{P}-\mathrm{C}}=13.6 \mathrm{~Hz}, 2 \mathrm{C}\right), 85.5(\mathrm{~s}, \mathrm{C}), 80.9\left(\mathrm{~d}, J_{\mathrm{P}-\mathrm{C}}=5.5 \mathrm{~Hz}\right.$, $\mathrm{CH}), 39.9\left(\mathrm{~s}, 2 \mathrm{CH}_{3}\right) \cdot{ }^{31} \mathrm{P}\left\{{ }^{1} \mathrm{H}\right\}$ NMR $\left(202 \mathrm{MHz}, \mathrm{CDCl}_{3}\right): \delta 27.79(\mathrm{~s})$. GC-MS (EI) m/z: 379 (22), 378 (4) (M) ${ }^{+}, 377$ (9), 362 (10), 292 (12), 281 (41), 253 (15), 208 (24), 207 (100), 193 (13), 191 (18), 135 (12), 133 (17), 95 (12), 73 (29). HRMS (ESI/Q-TOF) $\mathrm{m} / z$ : calcd for $\mathrm{C}_{22} \mathrm{H}_{19}$ ClNOP $[\mathrm{M}+\mathrm{H}]^{+}$, 380.0966; found, 380.0976 .
(2-Ethynyl-4-methoxyphenyl]diphenylphosphine Oxide (12d). Compound 5d (0.0525 g, $0.13 \mathrm{mmol})$ was reacted with $\mathrm{PhMgBr}$ $(0.195 \mathrm{~mL}, 0.195 \mathrm{mmol}, 1 \mathrm{M}$ solution in THF) according to general procedure E to afford $12 \mathrm{~d}(0.035 \mathrm{~g}, 0.107 \mathrm{mmol}, 82 \%$ yield) as a yellowish solid. $\mathrm{Mp}$ : $156.8-157.8{ }^{\circ} \mathrm{C} . R_{f}=0.15\left(10: 1 \quad \mathrm{CHCl}_{3} /\right.$ MTBE). ${ }^{1} \mathrm{H}$ NMR $\left(500 \mathrm{MHz}, \mathrm{CDCl}_{3}\right): \delta 7.72-7.76(\mathrm{~m}, 4 \mathrm{H}), 7.49-$ $7.56(\mathrm{~m}, 3 \mathrm{H}), 7.44-7.48(\mathrm{~m}, 4 \mathrm{H}), 7.13-7.14(\mathrm{~m}, 1 \mathrm{H}), 6.92-6.94$ $(\mathrm{m}, 1 \mathrm{H}), 3.86(\mathrm{~s}, 3 \mathrm{H}), 2.99(\mathrm{~s}, 1 \mathrm{H}) .{ }^{13} \mathrm{C}\left\{{ }^{1} \mathrm{H}\right\}$ NMR $(125 \mathrm{MHz}$, $\left.\mathrm{CDCl}_{3}\right): \delta 162.0\left(\mathrm{~d}, J_{\mathrm{P}-\mathrm{C}}=2.7 \mathrm{~Hz}, \mathrm{C}\right), 135.8\left(\mathrm{~d}, J_{\mathrm{P}-\mathrm{C}}=10.0 \mathrm{~Hz}\right.$, $\mathrm{CH}), 132.4\left(\mathrm{~d}, J_{\mathrm{P}-\mathrm{C}}=107.2 \mathrm{~Hz}, 2 \mathrm{C}\right), 132.2\left(\mathrm{~d}, J_{\mathrm{P}-\mathrm{C}}=10.0 \mathrm{~Hz}\right.$, $4 \mathrm{CH}), 131.7\left(\mathrm{~d}, J_{\mathrm{P}-\mathrm{C}}=2.7 \mathrm{~Hz}, 2 \mathrm{CH}\right), 128.3\left(\mathrm{~d}, J_{\mathrm{P}-\mathrm{C}}=11.8 \mathrm{~Hz}\right.$, $4 \mathrm{CH}), 126.9\left(\mathrm{~d}, J_{\mathrm{P}-\mathrm{C}}=8.2 \mathrm{~Hz}, \mathrm{C}\right), 125.7\left(\mathrm{~d}, J_{\mathrm{P}-\mathrm{C}}=107.2 \mathrm{~Hz}, \mathrm{C}\right)$, $120.3\left(\mathrm{~d}, J_{\mathrm{P}-\mathrm{C}}=10.0 \mathrm{~Hz}, \mathrm{CH}\right), 114.4\left(\mathrm{~d}, J_{\mathrm{P}-\mathrm{C}}=12.7 \mathrm{~Hz}, \mathrm{C}\right), 84.7(\mathrm{~s}$, C), $81.7\left(\mathrm{~d}, J_{\mathrm{P}-\mathrm{C}}=5.5 \mathrm{~Hz}, \mathrm{CH}\right), 55.5\left(\mathrm{~s}, \mathrm{CH}_{3}\right) .{ }^{31} \mathrm{P}\left\{{ }^{1} \mathrm{H}\right\} \operatorname{NMR}(202$ $\mathrm{MHz}, \mathrm{CDCl}_{3}$ ): $\delta 28.27$ (s). GC-MS (EI) $\mathrm{m} / \mathrm{z}: 333$ (22), 332 (100) $(\mathrm{M})^{+}, 331$ (76), 316 (11), 285 (13), 281 (15), 270 (18), 255 (48), 253 (24), 239 (90), 208 (15), 207 (34), 183 (28), 165 (31), 163 (11), 152 (15), 77 (36). HRMS (ESI/Q-TOF) $\mathrm{m} / z$ : calcd for $\mathrm{C}_{21} \mathrm{H}_{17} \mathrm{O}_{2} \mathrm{P}[\mathrm{M}+\mathrm{H}]^{+}, 333.1039$; found, 333.1040.

(2-Ethynyl-4-methoxyphenyl)phenyl[ $p$-( $N, N$-dimethylamino)phenyl]phosphine Oxide (12k). Compound 5d (0.0525 g, 0.13 mmol) was reacted with $p-\mathrm{Me}_{2} \mathrm{~N}-\mathrm{C}_{6} \mathrm{H}_{4} \mathrm{MgBr}(0.39 \mathrm{~mL}, 0.195 \mathrm{mmol}$, $0.5 \mathrm{M}$ solution in THF) according to general procedure $\mathrm{E}$ for $1 \mathrm{~h}$ to afford $12 \mathrm{k}(0.0311 \mathrm{~g}, 0.083 \mathrm{mmol}, 64 \%$ yield $)$ as an orange oil. $R_{f}=$ 0.57 (30:5:1 $\left.\mathrm{CHCl}_{3} / \mathrm{AcOEt} / \mathrm{MeOH}\right) .{ }^{1} \mathrm{H} \mathrm{NMR}\left(500 \mathrm{MHz}, \mathrm{CDCl}_{3}\right)$ : $\delta 7.69-7.73(\mathrm{~m}, 2 \mathrm{H}), 7.52-7.62(\mathrm{~m}, 4 \mathrm{H}), 7.40-7.47(\mathrm{~m}, 2 \mathrm{H}), 7.11-$ $7.12(\mathrm{~m}, 1 \mathrm{H}), 6.90-6.92(\mathrm{~m}, 1 \mathrm{H}), 6.69-6.71(\mathrm{~m}, 2 \mathrm{H}), 3.82(\mathrm{~s}, 3 \mathrm{H})$, $3.01(\mathrm{~s}, 6 \mathrm{H}), 2.99(\mathrm{~s}, 1 \mathrm{H}),{ }^{13} \mathrm{C}\left\{{ }^{1} \mathrm{H}\right\} \operatorname{NMR}\left(125 \mathrm{MHz}, \mathrm{CDCl}_{3}\right): \delta$ $161.7\left(\mathrm{~d}, J_{\mathrm{P}-\mathrm{C}}=2.7 \mathrm{~Hz}, \mathrm{C}\right), 152.3\left(\mathrm{~d}, J_{\mathrm{P}-\mathrm{C}}=2.7 \mathrm{~Hz}, \mathrm{C}\right), 135.8(\mathrm{~d}$, $\left.J_{\mathrm{P}-\mathrm{C}}=10.8 \mathrm{~Hz}, \mathrm{CH}\right), 133.9\left(\mathrm{~d}, J_{\mathrm{P}-\mathrm{C}}=107.2 \mathrm{~Hz}, \mathrm{C}\right), 133.6\left(\mathrm{~d}, J_{\mathrm{P}-\mathrm{C}}=\right.$ $10.9 \mathrm{~Hz}, 2 \mathrm{CH}), 132.2\left(\mathrm{~d}, J_{\mathrm{P}-\mathrm{C}}=10.0 \mathrm{~Hz}, 2 \mathrm{CH}\right), 131.2\left(\mathrm{~d}, J_{\mathrm{P}-\mathrm{C}}=2.7\right.$ $\mathrm{Hz}, \mathrm{CH}), 128.1\left(\mathrm{~d}, J_{\mathrm{P}-\mathrm{C}}=11.8 \mathrm{~Hz}, \mathrm{CH}\right), 127.3\left(\mathrm{~d}, J_{\mathrm{P}-\mathrm{C}}=106.3 \mathrm{~Hz}\right.$, C), $126.7\left(\mathrm{~d}, J_{\mathrm{P}-\mathrm{C}}=8.2 \mathrm{~Hz}, \mathrm{C}\right), 120.1\left(\mathrm{~d}, J_{\mathrm{P}-\mathrm{C}}=10.0 \mathrm{~Hz}, \mathrm{CH}\right), 117.0$ $\left(\mathrm{d}, J_{\mathrm{P}-\mathrm{C}}=118.1 \mathrm{~Hz}, \mathrm{C}\right), 114.2\left(\mathrm{~d}, J_{\mathrm{P}-\mathrm{C}}=12.7 \mathrm{~Hz}, 2 \mathrm{CH}\right), 111.2(\mathrm{~d}$, $\left.J_{\mathrm{P}-\mathrm{C}}=13.6 \mathrm{~Hz}, \mathrm{CH}\right), 84.3(\mathrm{~s}, \mathrm{C}), 81.7\left(\mathrm{~d}, J_{\mathrm{P}-\mathrm{C}}=5.5 \mathrm{~Hz}, \mathrm{CH}\right), 55.5$ $\left(\mathrm{s}, \mathrm{CH}_{3}\right), 39.9\left(\mathrm{~s}, 2 \mathrm{CH}_{3}\right) \cdot{ }^{31} \mathrm{P}\left\{{ }^{1} \mathrm{H}\right\} \mathrm{NMR}\left(202 \mathrm{MHz}, \mathrm{CDCl}_{3}\right): \delta 28.45$ (s). HRMS (ESI/Q-TOF) $m / z$ : calcd for $\mathrm{C}_{23} \mathrm{H}_{22} \mathrm{NO}_{2} \mathrm{P}[\mathrm{M}+\mathrm{H}]^{+}$, 376.1461; found, 376.1460 .

Reaction of $5 \mathrm{a}$ with $p$-TolMgBr at $-78{ }^{\circ} \mathrm{C}$ (General Procedure F). To a Schlenk tube $(25 \mathrm{~mL})$ equipped with a magnetic stirrer and an argon inlet was added phosphine oxide 5a $(0.0485 \mathrm{~g}, 0.13 \mathrm{mmol})$ in anhydrous THF $(2 \mathrm{~mL})$, and the mixture was cooled to $-78{ }^{\circ} \mathrm{C}$. Then, $p$-TolMgBr $(0.195 \mathrm{~mL}, 0.195 \mathrm{mmol}, 1$ $M$ solution in THF) was added. Then, the reaction mixture was stirred at $-78{ }^{\circ} \mathrm{C}$ for $30 \mathrm{~min}$. Then, the reaction mixture was quenched by the addition of $\mathrm{NH}_{4} \mathrm{Cl}$ solution $(5 \mathrm{~mL})$ and extracted with $\mathrm{CHCl}_{3}(5 \times 10 \mathrm{~mL})$. The collected organic phases were dried over $\mathrm{Na}_{2} \mathrm{SO}_{4}$, the solid was filtered off, and the filtrate was evaporated under reduced pressure. The crude reaction mixture was checked using the ${ }^{31} \mathrm{P}$ NMR technique. The residue was purified by column chromatography on silica gel using $\mathrm{CHCl}_{3} / \mathrm{EtOAc} / \mathrm{MeOH}(30: 5: 1 \mathrm{v} /$ v) to provide $8 \mathrm{a}(0.0148 \mathrm{~g}, 0.047 \mathrm{mmol}, 36 \%$ yield $)$ and 9 .

(2-Ethynylphenyl)(phenyl)((2-(phenyl(p-tolyl)phosphoryl)phenyl)ethynyl)phosphine Oxide (9): $0.0204 \mathrm{~g}, 0.038 \mathrm{mmol}, 29 \%$ yield, white solid (EtOAc). Mp: $233-234{ }^{\circ} \mathrm{C}(\mathrm{dec}) . R_{f}=0.27$ (30:5:1 $\left.\mathrm{CHCl}_{3} / \mathrm{AcOEt} / \mathrm{MeOH}\right) .{ }^{1} \mathrm{H}$ NMR $\left(500 \mathrm{MHz}, \mathrm{CDCl}_{3}\right): \delta 8.09-8.17$ $(\mathrm{m}, 1 \mathrm{H}), 7.78-7.82(\mathrm{~m}, 1 \mathrm{H}), 7.60-7.68(\mathrm{~m}, 4 \mathrm{H}), 7.45-7.75(\mathrm{~m}$, $10 \mathrm{H}), 7.33-7.43(\mathrm{~m}, 4 \mathrm{H}), 7.15-7.20(\mathrm{~m}, 2 \mathrm{H}), 3.20\left(\mathrm{~d}, J_{\mathrm{P}-\mathrm{H}}=1.89\right.$ $\mathrm{Hz}, 1 \mathrm{H}), 2.35\left(\mathrm{~d}, J_{\mathrm{P}-\mathrm{H}}=5.04 \mathrm{~Hz}, 3 \mathrm{H}\right) .{ }^{13} \mathrm{C}\left\{{ }^{1} \mathrm{H}\right\} \operatorname{NMR}(125 \mathrm{MHz}$, $\left.\mathrm{CDCl}_{3}\right): \delta 142.7\left(\mathrm{~d}, J_{\mathrm{P}-\mathrm{C}}=2.7 \mathrm{~Hz}, \mathrm{C}\right), 136.2\left(\mathrm{~d}, \mathrm{~J}_{\mathrm{P}-\mathrm{C}}=8.2 \mathrm{~Hz}, \mathrm{CH}\right)$, $135.4\left(\mathrm{~d}, J_{\mathrm{P}-\mathrm{C}}=99.9 \mathrm{~Hz}, \mathrm{C}\right), 134.4\left(\mathrm{dd}, J_{\mathrm{P}-\mathrm{C}}=1.8 \mathrm{~Hz}, J_{\mathrm{P}-\mathrm{C}}=11.8\right.$ $\mathrm{Hz}, \mathrm{CH}), 133.9\left(\mathrm{~d}, J_{\mathrm{P}-\mathrm{C}}=10.0 \mathrm{~Hz}, \mathrm{CH}\right), 133.4\left(\mathrm{~d}, J_{\mathrm{P}-\mathrm{C}}=10.0 \mathrm{~Hz}\right.$, $\mathrm{CH}), 132.2\left(\mathrm{~d}, J_{\mathrm{P}-\mathrm{C}}=3.6 \mathrm{~Hz}, \mathrm{CH}\right), 132.1\left(\mathrm{~d}, J_{\mathrm{P}-\mathrm{C}}=10.0 \mathrm{~Hz}, 2 \mathrm{CH}\right)$, $132.07\left(\mathrm{~d}, J_{\mathrm{P}-\mathrm{C}}=2.7 \mathrm{~Hz}, \mathrm{CH}\right), 132.06\left(\mathrm{~d}, J_{\mathrm{P}-\mathrm{C}}=3.08 \mathrm{~Hz}, \mathrm{CH}\right), 131.9$ $\left(\mathrm{d}, J_{\mathrm{P}-\mathrm{C}}=2.7 \mathrm{~Hz}, \mathrm{CH}\right), 131.85\left(\mathrm{dd}, J_{\mathrm{P}-\mathrm{C}}=4.5 \mathrm{~Hz}, J_{\mathrm{P}-\mathrm{C}}=124.4 \mathrm{~Hz}\right.$, C), $131.8\left(\mathrm{~d}, J_{\mathrm{P}-\mathrm{C}}=2.7 \mathrm{~Hz}, \mathrm{CH}\right), 131.7\left(\mathrm{~d}, J_{\mathrm{P}-\mathrm{C}}=1.8 \mathrm{~Hz}, \mathrm{CH}\right), 131.6$ $\left(\mathrm{d}, J_{\mathrm{P}-\mathrm{C}}=11.8 \mathrm{~Hz}, 2 \mathrm{CH}\right), 129.9\left(\mathrm{~d}, J_{\mathrm{P}-\mathrm{C}}=1.8 \mathrm{~Hz}, J_{\mathrm{P}-\mathrm{C}}=11.8 \mathrm{~Hz}\right.$, $\mathrm{CH}), 129.32\left(\mathrm{~d}, J_{\mathrm{P}-\mathrm{C}}=13.6 \mathrm{~Hz}, 2 \mathrm{CH}\right), 128.5\left(\mathrm{~d}, J_{\mathrm{P}-\mathrm{C}}=12.7 \mathrm{~Hz}\right.$, $2 \mathrm{CH}), 128.1\left(\mathrm{~d}, J_{\mathrm{P}-\mathrm{C}}=2.7 \mathrm{~Hz}, \mathrm{CH}\right), 128.1\left(\mathrm{~d}, J_{\mathrm{P}-\mathrm{C}}=2.7 \mathrm{~Hz}, \mathrm{CH}\right)$, $127.4\left(\mathrm{~d}, J_{\mathrm{P}-\mathrm{C}}=8.2 \mathrm{~Hz}, \mathrm{C}\right), 123.9(\mathrm{dm}, \mathrm{C}), 102.5\left(\mathrm{dd}, J_{\mathrm{P}-\mathrm{C}}=4.5 \mathrm{~Hz}\right.$, 
$\left.J_{\mathrm{P}-\mathrm{C}}=29.1 \mathrm{~Hz}, \mathrm{C}\right), 89.3\left(\mathrm{~d}, J_{\mathrm{P}-\mathrm{C}}=172.6 \mathrm{~Hz}, \mathrm{C}\right), 85.6(\mathrm{~s}, \mathrm{C}), 80.7$ $\left(\mathrm{dd}, J_{\mathrm{P}-\mathrm{C}}=2.7 \mathrm{~Hz}, J_{\mathrm{P}-\mathrm{C}}=7.3 \mathrm{~Hz}, \mathrm{CH}\right), 21.6\left(\mathrm{~s}, \mathrm{CH}_{3}\right) .{ }^{31} \mathrm{P}\left\{{ }^{1} \mathrm{H}\right\} \mathrm{NMR}$ $\left(202 \mathrm{MHz}, \mathrm{CDCl}_{3}\right): \delta 29.41\left(\mathrm{~d}, J_{\mathrm{P}-\mathrm{P}}=12.4 \mathrm{~Hz}\right), 7.44\left(\mathrm{~d}, J_{\mathrm{P}-\mathrm{P}}=12.4\right.$ $\mathrm{Hz}$ ). HRMS (ESI/Q-TOF) $m / z$ : calcd for $\mathrm{C}_{35} \mathrm{H}_{26} \mathrm{O}_{2} \mathrm{P}_{2}[\mathrm{M}+\mathrm{H}]^{+}$, 541.1481; found, 541.1479 .

Reaction of $5 \mathrm{a}$ with $p$-TolMgBr in a Higher Scale (General Procedure G). To a Schlenk tube $(25 \mathrm{~mL})$ equipped with a magnetic stirrer and an argon inlet phosphine was added oxide $5 \mathbf{a}(0.24 \mathrm{~g}, 0.634$ $\mathrm{mmol})$ in anhydrous THF (5 mL), and the mixture was cooled to 0 ${ }^{\circ} \mathrm{C}$. Then, $p$-TolMgBr $(0.952 \mathrm{~mL}, 0.952 \mathrm{mmol}, 1 \mathrm{M}$ in THF $)$ was added dropwise. Then, the reaction mixture was stirred at $0{ }^{\circ} \mathrm{C}$ for 1 h. Then, the reaction mixture was quenched by the addition of $\mathrm{NH}_{4} \mathrm{Cl}$ solution $(5 \mathrm{~mL})$ and extracted with $\mathrm{CHCl}_{3}(5 \times 5 \mathrm{~mL})$. The collected organic phases were dried over $\mathrm{Na}_{2} \mathrm{SO}_{4}$, the solid was filtered off, and the filtrate was evaporated under reduced pressure. The crude reaction mixture was checked using the ${ }^{31} \mathrm{P}\left\{{ }^{1} \mathrm{H}\right\}$ NMR technique. The residue was purified by column chromatography on silica gel using $\mathrm{CHCl}_{3} / \mathrm{EtOAc} / \mathrm{MeOH}(30: 5: 1 \mathrm{v} / \mathrm{v})$ as an eluent, yielding (2ethynylphenyl)phenyl( $p$-tolyl)phosphine oxide $(\mathbf{8 a})$ in $91 \%$ yield $(0.182 \mathrm{~g}, 0.577 \mathrm{mmol})$.

Reaction of 5a with PhLi or EtMgBr (General Procedure H). To a Schlenk tube $(25 \mathrm{~mL})$ equipped with a magnetic stirrer and an argon inlet was added phosphine oxide $5 \mathrm{a}(0.0405 \mathrm{~g}, 0.108 \mathrm{mmol})$ in anhydrous THF $(2 \mathrm{~mL})$, and the mixture was cooled to $0{ }^{\circ} \mathrm{C}$. Then, $\mathrm{PhLi}$ or EtMgBr $(0.164 \mathrm{mmol})$ was added. Then, the reaction mixture was stirred at $0{ }^{\circ} \mathrm{C}$ for $30 \mathrm{~min}$. Then, the reaction mixture was quenched by the addition of $\mathrm{NH}_{4} \mathrm{Cl}$ solution $(5 \mathrm{~mL})$ and extracted with $\mathrm{CHCl}_{3}(5 \times 10 \mathrm{~mL})$. The collected organic phases were dried over $\mathrm{Na}_{2} \mathrm{SO}_{4}$, the solid was filtered off, and the filtrate was evaporated under reduced pressure. The crude reaction mixture was checked using the ${ }^{31} \mathrm{P}\left\{{ }^{1} \mathrm{H}\right\}$ NMR technique. The residue was purified by column chromatography on silica gel using $\mathrm{CHCl}_{3} / \mathrm{MTBE}(30: 1 \mathrm{v} / \mathrm{v})$.

[2-(Ethynyl)phenyl]diphenylphosphine Oxide (8d). Compound 5a $(0.0405 \mathrm{~g}, 0.108 \mathrm{mmol})$ was reacted with $\mathrm{PhLi}(0.086 \mathrm{~mL}, 0.164$ mmol, $1.9 \mathrm{M}$ solution in $\left.n-\mathrm{Bu}_{2} \mathrm{O}\right)$ according to general procedure $\mathrm{H}$ to afford $8 \mathrm{~d}$ (0.007 g, $0.022 \mathrm{mmol}, 21 \%$ yield).

Ethyl(2-ethynylphenyl)phenylphosphine (13). Compound 5a $(0.0405 \mathrm{~g}, 0.108 \mathrm{mmol})$ was reacted with $\mathrm{EtMgBr}(0.162 \mathrm{~mL}$, $0.162 \mathrm{mmol}, 1 \mathrm{M}$ solution in THF) according to general procedure $\mathrm{H}$ to afford 13 (0.016 g, $0.063 \mathrm{mmol}, 58 \%$ yield) as a yellow oil. $R_{f}=$ 0.35 (30:5:1 $\left.\mathrm{CHCl}_{3} / \mathrm{AcOEt} / \mathrm{MeOH}\right) .{ }^{1} \mathrm{H} \mathrm{NMR}\left(500 \mathrm{MHz}, \mathrm{CDCl}_{3}\right)$ : $\delta 8.18-8.24(\mathrm{~m}, 1 \mathrm{H}), 7.71-7.76(\mathrm{~m}, 2 \mathrm{H}), 7.56-7.91(\mathrm{~m}, 1 \mathrm{H}), 7.46-$ $7.55(\mathrm{~m}, 3 \mathrm{H}), 7.40-7.44(\mathrm{~m}, 2 \mathrm{H}), 3.21(\mathrm{~s}, 1 \mathrm{H}), 2.71-2.80(\mathrm{~m}, 1 \mathrm{H})$, $2.51-2.61(\mathrm{~m}, 1 \mathrm{H}), 1.20\left(\mathrm{dt}, J_{\mathrm{H}-\mathrm{H}}=7.72 \mathrm{~Hz}, J_{\mathrm{H}-\mathrm{P}}=17.9 \mathrm{~Hz}, 3 \mathrm{H}\right)$. ${ }^{31} \mathrm{P}\left\{{ }^{1} \mathrm{H}\right\}$ NMR $\left(202 \mathrm{MHz}, \mathrm{CDCl}_{3}\right): \delta 34.50(\mathrm{~s}) .{ }^{13} \mathrm{C}\left\{{ }^{1} \mathrm{H}\right\} \mathrm{NMR}(125$ $\left.\mathrm{MHz}, \mathrm{CDCl}_{3}\right): \delta 134.7\left(\mathrm{~d}, J_{\mathrm{C}-\mathrm{P}}=8.62 \mathrm{~Hz}, \mathrm{CH}\right), 133.9\left(\mathrm{~d}, J_{\mathrm{C}-\mathrm{P}}=92.6\right.$ $\mathrm{Hz}, \mathrm{C}), 133.8$ (d, $\left.J_{\mathrm{C}-\mathrm{P}}=6.8 \mathrm{~Hz}, \mathrm{CH}\right), 133.1\left(\mathrm{~d} J_{\mathrm{C}-\mathrm{P}}=99.9 \mathrm{~Hz}, \mathrm{C}\right)$, $131.6\left(\mathrm{~d}, J_{\mathrm{C}-\mathrm{P}}=2.5 \mathrm{~Hz}, \mathrm{CH}\right), 131.5\left(\mathrm{~d}, J_{\mathrm{C}-\mathrm{P}}=2.5 \mathrm{~Hz}, \mathrm{CH}\right), 130.9(\mathrm{~d}$, $\left.J_{\mathrm{C}-\mathrm{P}}=9.99 \mathrm{~Hz}, 2 \mathrm{CH}\right), 128.9\left(\mathrm{~d}, J_{\mathrm{C}-\mathrm{P}}=10.0 \mathrm{~Hz}, \mathrm{CH}\right), 128.3\left(\mathrm{~d}, J_{\mathrm{C}-\mathrm{P}}\right.$ $=12.7 \mathrm{~Hz}, 2 \mathrm{CH}), 123.2\left(\mathrm{~d}, J_{\mathrm{C}-\mathrm{P}}=8.2 \mathrm{~Hz}, \mathrm{C}\right), 84.2(\mathrm{~s}, \mathrm{C}), 82.3(\mathrm{~d}$, $\left.J_{\mathrm{C}-\mathrm{P}}=4.5 \mathrm{~Hz}, \mathrm{CH}\right), 21.0\left(\mathrm{~d}, J_{\mathrm{C}-\mathrm{P}}=72.7 \mathrm{~Hz}, \mathrm{CH}_{2}\right), 5.59\left(\mathrm{~d}, J_{\mathrm{C}-\mathrm{P}}=5.5\right.$ $\mathrm{Hz}, \mathrm{CH}_{3}$ ). GC-MS (EI) $m / z$ : (\%) 255 (9), 254 (49) (M) ${ }^{+}, 253$ (9), 226 (35), 225 (100), 179 (17), 178 (30), 165 (17), 152 (12), 149 (11), 77 (25). HRMS (ESI/Q-TOF) $m / z$ : calcd for $\mathrm{C}_{32} \mathrm{H}_{30} \mathrm{O}_{2} \mathrm{P}_{2} \mathrm{Na}$ $[2 \mathrm{M}+\mathrm{Na}]^{+}, 531.1619$; found, 531.1628.

\section{ASSOCIATED CONTENT}

\section{(s) Supporting Information}

The Supporting Information is available free of charge at https://pubs.acs.org/doi/10.1021/acs.joc.1c01629.

${ }^{1} \mathrm{H}$ NMR, ${ }^{13} \mathrm{C}\left\{{ }^{1} \mathrm{H}\right\}$ NMR, ${ }^{31} \mathrm{P}\left\{{ }^{1} \mathrm{H}\right\}$ NMR, and ${ }^{19} \mathrm{~F}$ NMR spectra for all products, crystallographic data (PDF)

\section{Accession Codes}

CCDC 2094907 contains the supplementary crystallographic data for this paper. These data can be obtained free of charge via www.ccdc.cam.ac.uk/data_request/cif, or by emailing data_request@ccdc.cam.ac.uk, or by contacting The Cam- bridge Crystallographic Data Centre, 12 Union Road, Cambridge CB2 1EZ, UK; fax: +44 1223336033.

\section{AUTHOR INFORMATION}

\section{Corresponding Author}

Sylwia Sowa - Department of Organic Chemistry, Faculty of Chemistry, Institute of Chemical Sciences, Marie Curie-

Sklodowska University in Lublin, Lublin PL-20-614, Poland; ○ orcid.org/0000-0002-3494-1170; Email: sylwia.sowa@ poczta.umcs.lublin.pl

\section{Author}

Lukasz Ponikiewski - Department of Inorganic Chemistry, Faculty of Chemistry, Gdansk University of Technology, Gdansk PL-80-233, Poland; 이이.org/0000-0002-50371956

Complete contact information is available at: https://pubs.acs.org/10.1021/acs.joc.1c01629

\section{Notes}

The authors declare no competing financial interest.

\section{ACKNOWLEDGMENTS}

This work was supported by a grant from the National Centre of Science (Narodowe Centrum Nauki) in Poland (2018/02/ X/ST5/00635). S.S. thanks Prof. K. M. Pietrusiewicz for kindly reading the manuscript. Sylwia Sowa wish to thank Prof. Marek Stankevic for the discussions and for reading the manuscript.

\section{REFERENCES}

(1) (a) Stang, P. J.; Diederich, F. Modern Acetylene Chemistry; Wiley VCH, 1995; pp 1-26. (b) Diederich, F.; Stang, P. J.; Tykwinski, R. R. Acetylene Chemistry: Chemistry, Biology and Material Science; Wiley VCH, 2005; pp 1-42. (c) Trost, B. M.; Li, C.-J. Modern Alkyne Chemistry: Catalytic and Atom-Economic Transformations; Wiley VCH, 2015; pp 1-11.

(2) Trofimov, B. A.; Schmidt, E. Y. Acetylenes in the superbasepromoted assembly of carbocycles and heterocycles. Acc. Chem. Res. 2018, 51 (5), 1117-1130.

(3) (a) Jimenez-Nunez, E.; Echavarren, A. M. Molecular diversity through gold catalysis with alkynes. Chem. Commun. 2007, 333-346. (b) Uruvakili, A.; Gangadhararao, G.; Kumara Swamy, K. C. Gold(I) catalysed sequential dehydrative cyclisation/intermolecular $[4+2]$ cycloaddition of alkynyldienols onto activated alkynes/alkenes: a facile route to substituted norbornadienes/norbornenes. Org. Biomol. Chem. 2015, 13, 10060-10071. (c) Zhang, C.; Hong, K.; Dong, S.; Pei, C.; Zhang, X.; He, C.; Hu, W.; Xu, X. Gold(I)-catalyzed aromatization: expeditious synthesis of polyfunctionalized naphthalenes. iScience 2019, 21, 499-508.

(4) Tan, Q. W.; Chovatia, P.; Willis, M. C. Copper-catalysed synthesis of alkylidene 2-pyrrolinone derivatives from the combination of $\alpha$-keto amides and alkynes. Org. Biomol. Chem. 2018, 16, $7797-7800$.

(5) (a) Zhou, R.; Wang, W.; Jiang, Z.-J.; Wang, K.; Zheng, X.-L.; Fu, H.-Y.; Chen, H.; Li, R.-X. One-pot synthesis of 2-substituted benzo[b]furans via $\mathrm{Pd}$-tetraphosphine catalyzed coupling of 2halophenols with alkynes. Chem. Commun. 2014, 50, 6023-6026. (b) De, S.; Jash, M.; Chowdhury, C. Palladium(II) catalysed cascade strategy for the synthesis of dibenzo[5,6:7,8] cycloocta[1,2-b]indol10-ols/-10(15H)-ones: easy access to 1,3,5,7-cyclooctatetraenes (COTs). Chem. Commun. 2020, 56, 15659-15662. (c) Wang, J.; Wang, G.; Cheng, X.; Liu, Y.; Zhang, J. Sequential Sonogashira/ intramolecular aminopalladation/cross-coupling of ortho-ethynyl-anilines catalyzed by a single palladium source: rapid access to 2,3diarylindoles. Org. Biomol. Chem. 2021, 19, 1329-1333. 
(6) (a) Wan, S.-H.; Liu, S.-T. Functionalized pyrroles from vinylaziridines and alkynes via rhodium-catalyzed domino ringopening cyclization followed by $\mathrm{C}=\mathrm{C}$ bond migration. Tetrahedron 2019, 75, 1166-1170. (b) Burnie, A. J.; Evans, P. A. Rhodiumcatalyzed $[(3+2)+1]$ carbocyclizations of alkynylidenecyclopropanes with carbon monoxide: construction of polysubstituted bicyclohexa2,5-dienones. Chem. Commun. 2018, 54, 7621-7624.

(7) (a) Zhou, X.; Huang, F.; Tang, C.; Zhuo, Q.; Chen, Z.; Zhang, $\mathrm{H}$.; Xia, $\mathrm{H}$. A missing member of conjugated $\mathrm{N}$-heterocycles: realizing pyrido $[1,2-\alpha]$ azepine by reacting ruthenium alkenylcarbene complex with alkyne. Chem. Commun. 2018, 54, 4009-4012. (b) Ilies, L.; Arslanoglu, Y.; Matsubara, T.; Nakamura, E. Iron-catalyzed synthesis of indenones through cyclization of carboxamides with alkynes. Asian J. Org. Chem. 2018, 7 (7), 1327-1329. (c) Roldan-Molina, E.; Nievas, M. M.; Navarro, J. A. R.; Oltra, J. E. CpTiCl 2 -Catalyzed crosscoupling between Internal Alkynes and Ketones: A Novel Concept in the synthesis of halogenated, conjugated dienes. Chem. - Eur. J. 2020, 26 (37), 8296-8301.

(8) (a) Chang, X.; Zhang, J.; Peng, L.; Guo, C. Collective synthesis of acetylenic pharmaceuticals via enantioselective Nickel/Lewis acidcatalyzed propargylic alkylation. Nat. Commun. 2021, 12, 299. (b) Talele, T. T. Acetylene group, friend or foe in medicinal chemistry. J. Med. Chem. 2020, 63, 5625-5663.

(9) (a) Jiang, X.; Hao, X.; Jing, L.; Wu, G.; Kang, D.; Liu, X.; Zhan, P. Recent applications of click chemistry in drug discovery. Expert Opin. Drug Discovery 2019, 14 (8), 779-789. (b) Meldal, M.; Diness, F. Recent Fascinating Aspects of the CuAACClick Reaction. Trends in Chemistry 2020, 2 (6), 569-584. (c) Li, Y.; Wang, X.; Han, Y.; Sun, H.-Y.; Hilborn, J.; Shi, L. Click chemistry-based biopolymeric hydrogels for regenerative medicine. Biomed. Mater. 2021, 16, 022003.

(10) (a) Flaxman, H. A.; Miyamoto, D. K.; Woo, C. M. Small molecule interactome mapping by photo-affinity labeling (SIM-PAL) to identify binding sites of small molecules on a proteome-wide scale. Current Protocols in Chemical Biology 2019, 11, e75. (b) Croce, S.; Serdjukow, S.; Carell, T.; Frischmuth, T. Chemoenzymatic preparation of functional click-labeled messenger RNA. ChemBioChem 2020, 21 (11), 1641-1646. (c) Welling, M. M.; Duszenko, N.; van Willigen, D. M.; Hensbergen, A. W.; Buckle, T.; Rietbergen, D. D. D.; Roestenberg, M.; van Leeuwen, F. W. B. Interventional nuclear medicine: "click" chemistry as an in vivo targeting strategy for imaging microspheres and bacteria. Biomater. Sci. 2021, 9, 1683-1690.

(11) (a) Song, S.; Ko, Y.-G.; Lee, H.; Wi, D.; Ree, B. J; Li, Y.; Michinobu, T.; Ree, M. High-performance triazole-containing brush polymers via azide-alkyne click chemistry: a new functional polymer platform for electrical memory devices. NPG Asia Mater. 2015, 7, e228. (b) Marrocchi, A.; Facchetti, A.; Lanari, D.; Santoro, S.; Vaccaro, L. Click-chemistry approaches to $\pi$-conjugated polymers for organic electronics applications. Chem. Sci. 2016, 7, 6298-6308.

(12) (a) Mateos-Gil, J.; Calbo, J.; Rodríguez-Pérez, L.; Herranz, M. Á.; Ortí, E.; Martín, N. Carbon nanotubes conjugated with triazolebased tetrathiafulvalene-type receptors for $\mathrm{C}_{60}$ recognition. ChemPlusChem 2019, 84 (6), 730-739. (b) Sebastian, M.; Ganesan, A.; Behbehani, H.; Husain, A.; Makhseed, S. Ultrafast nonlinear optical characteristics of pyrene-conjugated azaphthalocyanines with optical limiting behavior. J. Phys. Chem. C 2020, 124, 21740-21750.

(13) (a) Ortiz, M.; Yu, C.; Jin, Y.; Zhang, W. Poly(aryleneethynylene)s: Properties, Applications and Synthesis Through Alkyne Metathesis. Top Curr. Chem. (Z) 2017, 375, 69. (b) Higashino, T.; Kurumisawa, Y.; Nimura, S.; Iiyama, H.; Imahori, H. Enhanced donor $-\pi$-acceptor character of a porphyrin dye incorporating naphthobisthiadiazole for efficient near-infrared light absorption. Eur. J. Org. Chem. 2018, 2018 (20-21), 2537-2547. (c) Mogensen, J.; Michaels, H.; Roy, R.; Broløs, L.; Kilde, M. D.; Freitag, M.; Nielsen, M. B. Indenofluorene-extended tetrathiafulvalene scaffolds for dyesensitized solar cells. Eur. J. Org. Chem. 2020, 2020 (38), 6127-6134. (d) Yenilmez, H. Y.; Sahin, A. N.; Altındal, A.; Bayır, Z. A. Photosensitive field effect transistor based on metallo-phthalocyanines containing (4-pentylphenyl)ethynyl moieties. Synth. Met. 2021, 273, 116690. (e) Sessler, J. L.; Wang, X.; Xie, L.; Lin, K.; Ma, W.; Zhao, T.;
Ji, X.; Khashab, N. M.; Alyami, M.; Wang, H. Calix[4]pyrrolecrosslinked porous polymeric networks for micropollutant removal from water. Angew. Chem., Int. Ed. 2021, 60 (13), 7188-7196. (f) Wu, Y.; Zang, Y.; Xu, L.; Wang, J.; Jia, H.; Miao, F. Synthesis of functional conjugated microporous polymer $/ \mathrm{TiO}_{2}$ nanocomposites and the mechanism of the photocatalytic degradation of organic pollutants. J. Mater. Sci. 2021, 56, 7936-7950.

(14) Zhang, Z.-H.; Dong, X.-Y.; Du, X.-Y.; Gu, Q.-S.; Li, Z.-L.; Liu, $\mathrm{X}$.-Y. Copper-catalyzed enantioselective Sonogashira-type oxidative cross-coupling of unactivated $\mathrm{C}\left(\mathrm{sp}^{3}\right)-\mathrm{H}$ bonds with alkynes. Nat. Commun. 2019, 10, 5689.

(15) Mohajer, F.; Heravi, M. M.; Zadsirjan, V.; Poormohammad, N. Copper-free Sonogashira cross-coupling reactions: an overview. RSC Adv. 2021, 11 (12), 6885-6925.

(16) (a) Yang, Q.; Zhou, Y.; Chen, J.; He, X.; Xu, J.; Kwong, F. Y.; Fan, B. Pd-Catalyzed allylic alkynylation of allylic acetates with terminal alkynes. Eur. J. Org. Chem. 2015, 2015 (24), 5330-5333. (b) Ha, H.; Shin, C.; Bae, S.; Joo, J. M. Divergent palladium-catalyzed cross-coupling of nitropyrazoles with terminal alkynes. Eur. J. Org. Chem. 2018, 2018 (20-21), 2645-2650. (c) Huang, W.-Y.; Lu, C.H.; Ghorai, S.; Li, B.; Li, C. Regio- and enantioselective allylic alkylation of terminal alkynes by synergistic $\mathrm{Rh} / \mathrm{Cu}$ catalysis. J. Am. Chem. Soc. 2020, 142 (36), 15276-15281. (d) Tsui, G. C.; Ma, Q.; Wang, Y. Stereoselective palladium-catalyzed C-F bond alkynylation of tetrasubstituted gem-difluoroalkenes. Angew. Chem., Int. Ed. 2020, 59 (28), 11293-11297. (e) Della-Felice, F.; Zanini, M.; Jie, X.; Tan, E.; Echavarren, A. M. Rhodium(III)-catalyzed synthesis of skipped enynes via $\mathrm{C}\left(\mathrm{sp}^{3}\right)-\mathrm{H}$ alkynylation of terminal alkenes. Angew. Chem., Int. Ed. 2021, 60 (11), 5693-5698. (f) Sun, M.; Liu, M.; Li, C. Rhodium-catalyzed chemodivergent regio- and enantioselective allylic alkylation of indoles. Chem. - Eur. J. 2021, 27 (10), 3457-3462.

(17) Rawe, B. W.; Scott, M. R.; Brown, C. M.; MacKenzie, H. K.; Gates, D. P. Poly(p-phenylenediethynylene phosphine)s and related pi-conjugated phosphine-diyne polymers: synthesis, characterization and photophysical Properties. Macromolecules 2017, 50, 8916-8927.

(18) Peng, L.; Xu, F.; Suzuma, Y.; Orita, A.; Otera, J. One-pot transformation of $\mathrm{Ph}_{2} \mathrm{P}(\mathrm{O})$-protected ethynes: deprotection followed by transition metal-catalyzed coupling. J. Org. Chem. 2013, 78 (24), 12802-12808.

(19) (a) Doherty, S.; Knight, J. G.; Perry, D. O.; Ward, N. A. B.; Bittner, D. M.; McFarlane, W.; Wills, C.; Probert, M. R. Triaryl-like MONO-, BIS-, and TRISKITPHOS phosphines: synthesis, solution NMR studies, and a comparison in gold-catalyzed carbon-heteroatom bond forming 5-exo-dig and 6-endo-dig cyclizations. Organometallics 2016, 35, 1265-1278. (b) Slowinski, F.; Aubert, C.; Malacria, M. J. Diastereoselective cobalt-mediated $[2+2+2]$ cycloadditions of substituted linear enediynes phosphine oxides: scope and limitations. J. Org. Chem. 2003, 68, 378-386.

(20) Melen, R. L.; Stephan, D. W. Cycloaddition reactions between dicyclohexylboron azide and alkynes. Dalton Trans. 2013, 42, 47954798.

(21) (a) Zhang, Y.; Hu, G.; Ma, D.; Xu, P.; Gao, Y.; Zhao, Y. TBAIcatalyzed oxidative $\mathrm{C}-\mathrm{H}$ functionalization: a new route to benzo[b]phosphole oxides. Chem. Commun. 2016, 52, 2815-2818. (b) Ma, D.; Pan, J.; Yin, L.; Xu, P.; Gao, Y.; Yin, Y.; Zhao, Y. Copper-catalyzed direct oxidative $\mathrm{C}-\mathrm{H}$ functionalization of unactivated cycloalkanes into cycloalkyl benzo[b] phosphole oxides. Org. Lett. 2018, 20, 34553459. (c) Liu, L.; Dong, J.; Yan, Y.; Yin, S.-F.; Han, L.-B.; Zhou, Y. Photoredox-catalyzed decarboxylative alkylation/cyclization of alkynylphosphine oxides: a metal- and oxidant-free method for accessing benzo[b]phosphole oxides. Chem. Commun. 2019, 55, 233-236.

(22) (a) Métivier, R.; Amengual, R.; Leray, I.; Michelet, V.; Genêt, J.-P. Novel fluorophores: efficient synthesis and photophysical properties. Org. Lett. 2004, 6 (5), 739-742. (b) Häussler, M.; Zheng, R.; Lam, J. W. Y.; Tong, H.; Dong, H.; Tang, B. Z. Hyperbranched polyynes: syntheses, photoluminescence, light refraction, thermal curing, metal complexation, pyrolytic ceramization, and soft magnetization. J. Phys. Chem. B 2004, 108, 10645-10650. (c) Ha-Thi, M.-H.; Souchon, V.; Hamdi, A.; Métivier, R.; Alain, V.; 
Nakatani, K.; Lacroix, P. G.; Genêt, J.-P.; Michelet, V.; Leray, I. Synthesis of novel rod-shaped and star-shaped fluorescent phosphane oxides-nonlinear optical properties and photophysical properties. Chem. - Eur. J. 2006, 12, 9056-9065. (d) Samb, I.; Bell, J.; Toullec, P. Y.; Michelet, V.; Leray, I. Fluorescent phosphane selenide as efficient mercury chemodosimeter. Org. Lett. 2011, 13 (5), 1182-1185.

(23) Qiao, S.; Huang, W.; Du, Z.; Chen, X.; Shieh, F.-K.; Yang, R. Phosphine oxide-based conjugated microporous polymers with excellent $\mathrm{CO}_{2}$ capture properties. New J. Chem. 2015, 39, 136-141.

(24) Biagiotti, G.; Langé, V.; Ligi, C.; Caporali, S.; Muniz-Miranda, M.; Flis, A.; Pietrusiewicz, K. M.; Ghini, G.; Brandi, A.; Cicchi, S. Nanostructured carbon materials decorated with organophosphorus moieties: synthesis and application. Beilstein J. Nanotechnol. 2017, 8, 485-493.

(25) (a) Smith, C. D.; Baxendale, I. R.; Tranmer, G. K.; Baumann, M.; Smith, S. C.; Lewthwaite, R. A.; Ley, S. V. Tagged phosphine reagents to assist reaction work-up by phase-switched scavenging using a modular flow reactor. Org. Biomol. Chem. 2007, 5, 15621568. (b) Maugeri, L.; Lebl, T.; Cordes, D. B.; Slawin, A. M. Z.; Philp, D. Cooperative binding in a phosphine oxide-based halogen bonded dimer drives supramolecular oligomerization. J. Org. Chem. 2017, 82, 1986-1995.

(26) (a) Winter, W. Photochemische cyclisierung von $o, o^{\prime}-$ bis(phenyläthinyl)-triphenylphosphin zum phosphindol-system. Tetrahedron Lett. 1975, 16, 3913-3914. (b) Butters, T.; Winter, W. Synthese von Triphenylphosphan-en-inen und Phosphindolen sowie Röntgenstrukturanalyse von zwei Phosphindolen. Chem. Ber. 1984, 117, 990-1002. (c) Tsuji, H.; Sato, K.; Ilies, L.; Itoh, Y.; Sato, Y.; Nakamura, E. Modular synthesis of benzo[b]phosphole derivatives via BuLi-mediated cyclization of (o-alkynylphenyl)phosphine. Org. Lett. 2008, 10 (11), 2263-2265. (d) Sanji, T.; Shiraishi, K.; Kashiwabara, T.; Tanaka, M. Base-mediated cyclization reaction of 2-alkynylphenylphosphine oxides: synthesis and photophysical properties of benzo[b]phosphole oxides. Org. Lett. 2008, 10 (13), 2689-2692. (e) Xu, Y.; Wang, Z.; Gan, Z.; Xi, Q.; Duan, Z.; Mathey, F. Versatile synthesis of phospholides from open-chain precursors. Application to annelated pyrrole- and silole-phosphole rings. Org. Lett. 2015, 17 (7), 1732-1734. (f) Zhao, X.; Gan, Z.; Hu, C.; Duan, Z.; Mathey, F. Planar polycyclic oxaphosphoranes incorporating a benzophosphole unit. Org. Lett. 2017, 19, 5814-5817.

(27) (a) Winter, W. 2,2'-Bis(diphenylphosphino)-2',3,5',6'-tetraphenylpterphenyl als Aren-Chelat-Ligand fur Rhodium(1). Angew. Chem. 1976, 88, 260. (b) Vedejs, E.; Steck, P. L. Unusual oxaphosphoranes by acyl transfer from $o$-acetoxy- $o^{\prime}$-diphenylphosphanyltolane. Angew. Chem., Int. Ed. 1999, 38 (18), 2788-2791. (c) Song, Y.; Wang, L.; Duan, Z.; Mathey, F. Divergent intramolecular reactions between phosphines and alkynes. Chin. Chem. Lett. 2020, 31 (2), 329-332.

(28) (a) Belmonte Sánchez, E.; Iglesias, M. J.; El Hajjouji, H.; Roces, L.; García-Granda, S.; Villuendas, P.; Urriolabeitia, E. P.; Ortiz, L. F. Cycloaurated phosphinothioic amide complex as a precursor of gold(I) nanoparticles: efficient catalysts for $\mathrm{A}^{3}$ synthesis of propargylamines under solvent-free conditions. Organometallics 2017, 36 (10), 1962-1973. (b) Mahamulkar, S. G.; Císǎrová, I.; Jahn, U. New phosphine ligand architectures lead to efficient gold catalysts for cycloisomerization reactions at very low loading. Adv. Synth. Catal. 2018, 360 (21), 4215-4224.

(29) Hu, W.; Li, E.-Q.; Duan, Z.; Mathey, F. Concise synthesis of phospholene and its P-stereogenic derivatives. J. Org. Chem. 2020, 85, 14772-14778.

(30) Doherty, S.; Knight, J. G.; Smyth, C. H.; Harrington, R. W.; Clegg, W. Rhodium-catalyzed double $[2+2+2]$ cycloaddition of $1,4-$ bis(diphenylphosphinoyl)buta-1,3-diyne with tethered diynes: A modular, highly versatile single-pot synthesis of NU-BIPHEP biaryl diphosphines. Org. Lett. 2007, 9 (23), 4925-4928.

(31) (a) Peng, A.-Y.; Zhang, X.-Y.; Ding, Y.-X. A convenient and applicable route to synthesize 2-(1-alkynyl)phenylphosphonates. Heteroat. Chem. 2005, 16 (6), 529-534. (b) Peng, A.-Y.; Ding, Y.$\mathrm{X}$. Synthesis of phosphaisocoumarins via iodocyclization. Org. Lett.
2004, 6 (7), 1119-1121. (c) Peng, A.-Y.; Ding, Y.-X. The synthesis of phosphaisocoumarins by $\mathrm{Cu}(\mathrm{I})$-catalyzed intramolecular cyclization of o-ethynylphenylphosphonic acid monoesters. J. Am. Chem. Soc. 2003, 125, 15006-15007. (d) Peng, A.-Y.; Ding, Y.-X. Synthesis of 4halophosphaisocoumarins via halocyclization of 2-(1-alkynyl)phenylphosphonates. Tetrahedron 2005, 61, 10303-10308.

(32) Xiao, Z.; Peng, A.-Y. Palladium(II) acetate catalyzed cyclization-coupling of (o-ethynylphenyl)phosphonic acid monoesters with allyl halides. Synthesis 2019, 51 (18), 3499-3505.

(33) Sheldrick, G. A short history of SHELX. Acta Crystallogr., Sect. A: Found. Crystallogr. 2008, 64, 112-122.

(34) Farrugia, L. WinGX and ORTEP for Windows: an update. J. Appl. Crystallogr. 2012, 45, 849-854.

(35) Stankevič, M.; Włodarczyk, A. Efficient copper(I)-catalyzed coupling of secondary phosphine oxides with aryl halides. Tetrahedron 2013, 69 (1), 73-81.

(36) Azevedo, C. M. G.; Afonso, C. M. M.; Soares, J. X.; Reis, S.; Sousa, D.; Lima, R. T.; Vasconcelos, M. H.; Pedro, M.; Barbosa, J.; Gales, L.; Pinto, M. M. M. Pyranoxanthones: Synthesis, growth inhibitory activity on human tumor cell lines and determination of their lipophilicity in two membrane models. Eur. J. Med. Chem. 2013, 69, 798-816.

(37) Masurier, N.; Estour, F.; Froment, M.-T.; Lefevre, B.; Debouzy, J.-C.; Brasme, B.; Masson, P.; Lafont, O. Synthesis of 2-substituted $\beta$ cyclodextrin derivatives with a hydrolytic activity against the organophosphorylester paraoxon. Eur. J. Med. Chem. 2005, 40 (7), 615-623.

(38) Kawai, J.; Chikkade, K. P.; Shimizu, Y.; Kanai, M. In situ catalytic generation of allylcopper species for asymmetric allylation: Toward 1H-isochromene skeletons. Angew. Chem., Int. Ed. 2013, 52 (28), 7177-7180.

(39) Mülbaier, M.; Giannis, A. First synthesis and oxidative properties of polymer-supported IBX. ARKIVOC 2003, 2003, 228236.

(40) Nakazaki, A.; Mori, A.; Kobayashi, S.; Nishikawa, T. A Divergent approach to the diastereoselective synthesis of 3,3disubstituted oxindoles from atropisomeric $\mathrm{N}$-aryl oxindole derivatives. Chem. - Asian J. 2016, 11 (22), 3267-3274.

(41) Gavara, L.; Petit, C.; Montchamp, J.-L. DBU-promoted alkylation of alkyl phosphinates and H-phosphonates. Tetrahedron Lett. 2012, 53, 5000-5003. 